\title{
Weißbuch \\ Physikalische und Rehabilitative Medizin \\ in Europa
}

Erstellt von der Section of Physical and Rehabilitation Medicine,

Union Européenne des Médecins Spécialistes (UEMS)

in Zusammenarbeit mit der Académie Européenne de Médecine de Réadaptation

und der European Society for Physical and Rehabilitation Medicine

\author{
Herausgeber: \\ Christoph Gutenbrunner \\ Chairman, Professional Practice Committee, UEMS Section of Physical \& Rehabilitation Medicine \\ Anthony B. Ward \\ President, UEMS Section of Physical \& Rehabilitation Medicine \\ M. Anne Chamberlain \\ President, Académie Européenne de Médecine de Réadaptation
}

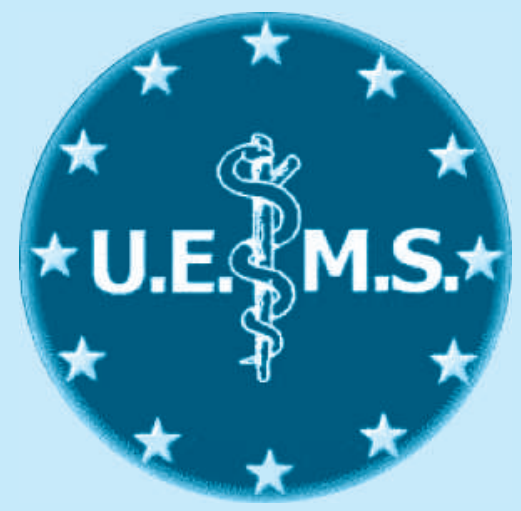

September 2006

mit Beiträgen von

Prof. André Bardot $(\mathrm{F})$

Prof. Michel Barat (F)

Dr. Laurent Bensoussan (F)

Prof. Mihai Berteanu (Ro)

Dr. Pedro Cantista (P)

Prof. Anne Chamberlain (UK)

Dr. Nicolas Christodolou (Cy)

Dr. Alarcos Cieza (D)

Prof. Alain Delarque (F)

Prof. Jean-Pierre Didier (F)

Prof. Veronika Fialka-Moser (A)

Prof. Franco Franchignoni (I)

Prof. Alessandro Giustini (I)

Das englische Original wird in J Rehab Med publiziert
Prof. Christoph Gutenbrunner (D)

Prof. Gustaaf Lankhorst (NL)

Prof. Lindsay McLellan (UK)

Dr. Fernando Parada (P)

Prof. João Páscoa Pinheiro (P)

Prof. Michael Quittan (A)

Prof. Bengt Sjölund (S)

Prof. Henk Stam (NL)

Prof. Gerold Stucki (D)

Prof. Guy Vanderstraeten (B)

Dr. Jiri Votava $(\mathrm{Cz})$

Prof. Jean-Michel Viton (F)

Dr. Anthony Ward (UK)

Übersetzung aus dem Englischen: Sujata Wagner, Leobendorf, Österreich 
Dieses Buch beschreibt die Stellung der Physikalischen und Rehabilitativen Medizin (PRM) in Europa. Es definiert das Fachgebiet, sein Arbeitsfeld, die Kompetenzen der Fachärzte und die Beziehungen zu anderen medizinischen Fachgebieten und Gesundheitsberufen. Das Buch soll auch dazu beitragen, dass die PRM auf europäischer Ebene als eigenständige Fachdisziplin anerkannt wird, in der hoch qualifizierte, an anerkannten Behandlungsstandards orientierte Fachärzte auf wissenschaftlicher Basis und im Kontext der unterschiedlichen nationalen Systeme arbeiten können. Zu diesem Zweck werden Ausbildung und Fertigkeiten der PRM-Fachärzte im Detail beschrieben. Darüber hinaus werden die Grundprinzipien der speziellen Rehabilitation dargestellt, mit dem Ziel, politischen Entscheidungsträgern, Planern im Gesundheitswesen, medizinischen und nichtmedizinischen Rehabilitationsberufen einen Einblick in die Funktionsweise der PRM zu geben und aufzuzeigen, wie sie den Rehabilitationsprozess fördern und Menschen mit Behinderungen eine uneingeschränkte Teilnahme am gesellschaftlichen Leben ermöglichen kann.

Das Buch wurde von der UEMS Section of Physical and Rehabilitation Medicine unter der Ägide des Präsidenten und des Vorsitzenden des Professional Practice Committee sowie der Präsidentin der Académie Européenne de Médecine de Réadaptation herausgegeben. Es wurde von allen drei Körperschaften angenommen, die das Fachgebiet in Europa vertreten, namentlich der Section for
Physical and Rehabilitation Medicine der Union Européenne des Médecins Spécialistes, der Académie Européenne de Médecine de Réadaptation und der European Society of Physical and Rehabilitation Medicine. Hierdurch erhält das Buch ein hohes Gewicht.

Die Herausgeber erhielten Beiträge aus ganz Europa, haben die Texte jedoch im Interesse einer einheitlichen Präsentation und Herangehensweise bearbeitet. Besonderer Wert wurde darauf gelegt, dass das Weißbuch unter Berücksichtigung der einzelstaatlichen Unterschiede dem gesamteuropäischen System Rechnung trägt. Der besondere Dank der Herausgeber gilt allen Mitarbeitern und Mitarbeiterinnen für ihr bemerkenswertes Bemühen, ein wirklich paneuropäisches Werk zu schaffen. Ihre Namen sind oben in alphabetischer Reihenfolge aufgelistet.

Die Originalfassung des Weißbuchs ist über die Webseite der Section (www.euro-prm.org) oder über das Generalsekretariat der UEMS PRM-Section erhältlich.

Anthony B. Ward, Präsident, UEMS PRM-Section

Christoph Gutenbrunner, Chairman, Professional Practice Committee

M. Anne Chamberlain, Präsidentin, Académie 
Zusammenfassung (Executive Summary) 4

1 Einleitung 7

2 Definitionen 7

2.1 Rehabilitation 7

2.2 Physikalische und Rehabilitative Medizin 7

3 Relevanz der Rehabilitation für Menschen mit Behinderungen und für die Gesellschaft 8

3.1 Epidemiologische Aspekte 8

3.2 Das WHO-Modell der Funktionsfähigkeit, Behinderung und Gesundheit in der Rehabilitation 9

3.3 Ethische Aspekte und Menschenrechte 10

3.4 Rehabilitation und Gesundheitssysteme 13

3.5 Ziele und Ergebnisse der Rehabilitation 13

4 Grundlagen der Physikalischen und Rehabilitativen Medizin 14

4.1 Lernprozesse als Grundlage der Physikalischen und Rehabilitativen Medizin 14

4.2 Ziele der Physikalischen und Rehabilitativen Medizin 14

4.3 Das Rehabilitationsteam 14

4.4 Physikalische und Rehabilitative Medizin in unterschiedlichen Phasen des Rehabilitationsprozesses 15

4.5 Auswirkungen einer fehlenden Rehabilitation 16

4.6 Prävention 17

5 Das Fachgebiet der Physikalischen und Rehabilitativen Medizin 18

5.1 Beitrag des Facharztes für Physikalische und Rehabilitative Medizin zum Rehabilitationsprozess 18

5.2 Das Fachgebiet der Physikalischen und Rehabilitativen Medizin in Europa 18

5.3 Krankheiten und Funktionssstörungen, die in der Physikalischen und Rehabilitativen Medizin behandelt werden 18

5.4 Diagnostik, Assessment und Evaluation 19

5.5 Rehabilitationsplan 20

5.6 Interventionen der Physikalischen und Rehabilitativen Medizin 20

5.7 Physikalische und Rehabilitative Medizin in der Praxis - klinische Tätigkeiten und Einrichtungen 21

6 Qualitätsstandards in der Physikalischen und Rehabilitativen Medizin 22

6.1 Theoretische und praktische Ausbildung 22

6.2 Klinische Qualitätsstandards und Kompetenzen des Facharztes für Physikalische und Rehabilitative Medizin 23

6.3 Kontinuierliche berufliche Entwicklung (CPD) und medizinische Fortbildung (CME) 24

6.4 Sektion Physikalische und Rehabilitative Medizin der Europäischen Union der Fachärzte (UEMS) 24

6.5 Académie Européenne de Médecine de Réadaptation 26

6.6 Europäische Gesellschaft für Physikalische und Rehabilitative Medizin (ESPRM) 26

7 Forschung auf dem Gebiet der Physikalischen und Rehabilitativen Medizin 27

7.1 Bedeutung der Forschung zur Ermittlung des Rehabilitationsbedarfs und Wert jetziger und zukünftiger Ansätze 27

7.2 Forschungseinrichtungen und -ressourcen 27

7.3 Ausbildung in der Forschung 28

7.4 Veröffentlichung der Forschungsergebnisse 28

8 Zukünftige Entwicklungen 28

8.1 Grundsätze 28

8.2 Ziele 28

9 Literatur 28

Anhang 1a Vorschlag für eine neue Definition basierend auf dem ICF-Modell 30

Anhang 1b Beschreibung des Fachgebietes der PRM 31

Anhang 2 Demografische Daten zu den PRM-Fachärzten in den Mitgliedstaaten der UEMS 31

Anhang 3 Beispiele für zu Behinderungen führenden Erkrankungen, mit denen PRM-Fachärzte häufig konfrontiert sind 32

Anhang $4 \quad$ Diagnostische Methoden und Assessments in der Physikalischen und Rehabilitativen Medizin 32

Anhang $5 \quad$ Curriculum und theoretisches Wissen für ein PRM-Diplom des Europäischen Boards (Version 2005) 33

Anhang 6 Regeln für die kontinuierliche berufliche Entwicklung (CME) und medizinische Fortbildung

von PRM-Fachärzten $\quad 36$

Danksagungen 


\section{Einleitung}

1. Dieses Buch richtet sich an:

- politische Entscheidungsträger im Gesundheitssektor, die sich mit Fragen der Rehabilitation und Behinderung befassen;

- die allgemeine Öffentlichkeit, insbesondere an Menschen mit Behinderungen sowie an Vertreter ihrer Organisationen sowie

- Fachleute anderer medizinischer Fachgebiete und Gesundheitsberufen

2. Es beschreibt die Charakteristika, Arbeitsbereiche und Parameter der Physikalischen und Rehabilitativen Medizin (PRM), die Kompetenzen des Fachgebiets und seiner Fachärzte, den Inhalt der klinischen Arbeit im Gebiet und die Merkmale der Aus- und Weiterbildung. Das „Gemeinsame Europa“ wurde in letzter Zeit wieder erweitert, und dieses Buch möchte einen Beitrag zur Harmonisierung der Tätigkeit der PRM-Fachärzte leisten und damit erreichen, dass die Bevölkerung und insbesondere die Menschen mit Behinderungen unabhängig von ihrem Wohnort optimal betreut werden.

\section{Definitionen}

1. Die Weltgesundheitsorganisation (WHO) definiert den Begriff Rehabilitation wie folgt: „Die Anwendung aller Mittel mit dem Ziel, die Auswirkungen behindernder und einschränkender Bedingungen zu mildern und Menschen mit Behinderungen eine optimale soziale Integration zu ermöglichen.“

2. Gemäß Definition der Union Européenne des Medécins Spécialistes (UEMS) Section of PRM ist die Physikalische und Rehabilitative Medizin „ein unabhängiges medizinisches Fachgebiet, das sich mit der Förderung der körperlichen und kognitiven Funktionsfähigkeit, Aktivitäten (einschließlich Verhalten) und Teilhabe (einschließlich Lebensqualität) sowie der Modifizierung von persönlichen und Umweltfaktoren befasst. Es ist somit für die Prävention, die Diagnostik, Behandlung und das Rehabilitationsmanagement von Menschen mit krankheitsbedingten Funktionseinschränkungen und Komorbiditäten in allen Altersgruppen“ zuständig.

\section{Relevanz der Rehabilitation für Menschen mit Behinderungen und für die Gesellschaft}

1. Die Prävalenz von Behinderungen wird für die meisten europäischen Länder übereinstimmend bei etwa $10 \%$ angegeben. Aufgrund der zunehmenden Alterung der Bevölkerung nimmt auch der Anteil an Menschen mit Behinderungen zu. Dies bedingt einen erhöhten Versorgungsbedarf für den Einzelnen. Für die Gesellschaft bedeutet dies steigende finanzielle Aufwendungen für Gesundheit und soziale Sicherung. Die Überlebensraten nach schweren Krankheiten und Verletzungen steigen zwar, wodurch auch die Zahl von Menschen mit oft komplexen Funktionsstörungen zunimmt. Hinzu kommt, dass die Menschen in Europa immer höhere Ansprüche an die Aufrechterhaltung eines guten Gesundheitszustands stellen.

Die Rehabilitation reduziert wirksam die mit Behinderungen einhergehenden Belastungen und erweitert die Möglichkeiten der Betroffenen. Die Kosten der Rehabilitation sind in der Regel nicht höher als für die Gesundheitsleistungen, die ohne Rehabilitation notwendig gewesen wären. Wenn es gelingt,
Folgestörungen von Immobilität, ZNS-Schädigungen und Schmerzen zu verhindern (wofür es eindeutige Evidenz gibt), hat dies für den Einzelnen große qualitative und für die Gesellschaft quantitative, sprich finanzielle Vorteile.

2. Das biopsychosoziale Modell der Behinderung. Die PRM verfolgt methodisch einen biopsychosozialen Ansatz in der Rehabilitation. Dieser wurde in Zusammenarbeit mit Anwenderorganisationen entwickelt und basiert auf der International Classification of Functioning Disability and Health (ICF - Internationale Klassifikation der Funktionsfähigkeit, Behinderung und Gesundheit) der WHO, die im Mai 2001 von der World Health Assembly (WHA - Weltgesundheitsversammlung) verabschiedet worden ist. Diese Klassifikation ist ätiologisch neutral und verwendet eine weltweit anerkannte Terminologie zur Abgrenzung von Funktionsfähigkeiten auf individueller und auf Bevölkerungsebene. Es eignet sich hervorragend für das Management individueller Rehabilitationsprogramme und Interventionen. Im Rahmen der ICF können die zugrunde liegenden Pathologien, die Probleme auf der Ebene der Funktionsfähigkeit der Organe beschreiben und das Potenzial für eine Wiederherstellung bzw. Optimierung der persönlichen Funktionsfähigkeit oder der Verhinderung weiterer Aktivitätsbeschränkungen identifiziert werden. Zusätzlich werden die Fähigkeiten, am gesellschaftlichen Leben teil $\mathrm{zu}$ haben, berücksichtigt. Sie sind nicht nur von der persönlichen Funktionsfähigkeit, sondern auch von kontextbedingten, das Leben beeinflussenden Faktoren und der Umwelt der Betroffenen abhängig.

3. Ethische Aspekte und Menschenrechte. Der Zugang zu Rehabilitationsleistungen ist ein grundlegendes Menschenrecht, das in der Charta der Vereinten Nationen über ihre Standards (1993), anlässlich des Europäischen Jahres der Menschen mit Behinderungen (2003) vom Europäischen Rat und in der 58. Resolution der Weltgesundheitsversammlung (2005) verankert ist. Darüber hinaus sind in vielen europäischen Ländern Antidiskriminierungsgesetze in Kraft, auf die sich Menschen mit Behinderungen, deren Familien und Helfer berufen können. Die PRM-Fachärzte sind bei der Betreuung ihrer Patienten selbstverständlich in die Diskussionen über ethische und rechtliche Probleme mit eingebunden.

Der gleichberechtigte Zugang zu Rehabilitation und sozialer Teilhabe ohne jegliche Art von Diskriminierung sind fundamentale Grundsätze in der Bereitstellung und Durchführung der Rehabilitation. Die PRM-Fachärzte sind sich der Belastungen bewusst, denen Menschen aufgrund unterschiedlicher Rassen, Kultur, Religion und sexueller Ausrichtung ausgesetzt sind. Die Rehabilitation hat das Ziel, den Menschen ein unabhängiges Leben zu ermöglichen und ihre Autonomie zu fördern. Bei der Umsetzung dieses Ziels kommt ein ganzheitlicher Ansatz zur Anwendung.

Die PRM ist für alle gesellschaftlichen Segmente in Europa von Bedeutung. Ihre Vertreter unterstützen die Erklärungen des Internationalen Jahres für Menschen mit Behinderungen (1981) und des Europäischen Jahres für Menschen mit Behinderungen (2003), denen zufolge der Zugang zu Rehabilitation nach Verletzung oder Krankheit ein grundlegendes Menschenrecht ist.

4. Menschen mit Behinderungen sollten an der Schaffung und Entwicklung von Rehabilitationsangeboten aktiv teilnehmen. Bei der Rehabilitation im Sinne der „Good Clinical Practice“ stehen die Menschen mit Behinderungen im Mittelpunkt 
eines multidisziplinären Ansatzes. Sie sollen in die Lage versetzt werden, aufgrund umfassender Informationen eine selbstständige Wahl der Behandlung zu treffen. Gegebenenfalls sollen auch ihre Familien in den Rehabilitationsprozess einbezogen werden.

5. Ziele und Ergebnisse der Rehabilitation. Das Gesamtziel der Rehabilitation besteht darin, Menschen mit Behinderungen in die Lage zu versetzen, das von ihnen gewünschte Leben zu führen. Dabei sind naturgemäß die Einschränkungen der Handlungs- und Aktivitätsmöglichkeiten aufgrund der Funktionsbeeinträchtigungen in Rechnung zu stellen, die sich aus Krankheit oder Verletzung oder aus ihren persönlichen Lebensverhältnissen ergeben. In der Praxis wird dies oftmals am besten mit einer Kombination von Maßnahmen erreicht, die das Ziel haben, die Beeinträchtigungen zu überwinden oder bestmöglich zu handhaben bzw. Barrieren abzubauen, die einer Teilhabe an den von der betreffenden Person gewählten Lebensbereichen entgegenstehen. Ein derartiger Prozess zielt auf eine Optimierung von Aktivität und Teilhabe. Die zwei grundlegenden Ergebnisse einer Rehabilitation, die es nachzuweisen gilt, sind das Wohlbefinden der Person und ihre soziale und berufliche Teilhabe.

\section{Grundlagen der Physikalischen und Rehabilitativen Medizin}

1. Lernprozesse sind unter modernen Gesichtspunkten einer der wichtigsten Teile der Rehabilitation. Der PRM-Facharzt übernimmt die Funktion eines Lehrers, insbesondere wenn die neuen Konzepte von Plastizität und motorischem Lernen eingesetzt werden. PRM-Fachärzte müssen die Grundlagen von Adaptation und Plastizität kennen und über den theoretischen Hintergrund der Grundlagen von Lehren und Lernen Bescheid wissen.

2. Die Physikalische und Rehabilitative Medizin zielt darauf ab, krankheitsbedingte Beeinträchtigungen nach Möglichkeit zu reduzieren, und zwar dardurch, dass Krankheitskomplikationen verhindert, die Funktionsfähigkeit und Aktivitäten verbessert werden sowie eine Teilhabe am Leben in der Gemeinschaft ermöglicht wird. Alle diese Aktivitäten müssen den persönlichen, kulturellen und Umweltkontext der/des Betroffenen berücksichtigen. Die praktische Umsetzung findet in unterschiedlichen Einrichtungen statt; diese reichen von der Akutversorgung bis hin zu gemeindenahen Versorgungseinrichtungen. Die PRM-Fachärzte verwenden spezifische diagnostische Untersuchungsmethoden und führen zahlreiche unterschiedliche Therapien durch, darunter pharmakologische, physikalische, technische, aufklärungsbezogene und berufsbezogene Interventionen. Die Rehabilitation ist ein kontinuierlicher und koordinierter Prozess, der mit dem Beginn einer Krankheit oder einer Verletzung einsetzt und so lange dauert, bis die/der Betroffene eine Rolle in der Gesellschaft erreicht hat, die seinen bzw. ihren Lebenszielen und Wünschen entspricht.

3. Rehabilitationsleistungen werden in multidisziplinären Teams aus Angehörigen verschiedener medizinischer Fachberufe erbacht, die koordiniert, zielorientiert und patientenzentriert arbeiten. Geleitet werden diese Teams in spezialisierten PRM-Einrichtungen in der Regel von PRM-Fachärzten, die auch für die medizinische Betreuung ihrer Patienten verantwortlich sind. PRM-Fachärzte arbeiten auch eng mit Vertretern anderer medizinischer Fachgebiete zusammen und übernehmen in den Fällen, in denen die Rehabilitation in den Mittelpunkt der klinischen Maßnahmen rückt, auch die Leitung dieser fachübergreifenden Kooperation.

4. Physikalische und Rehabilitative Medizin in unterschiedlichen Phasen des Rehabilitationsprozesses. Die Rehabilitation kann in einer Reihe unterschiedlicher Einrichtungen durchgeführt werden, wobei das Spektrum von spezialisierten Rehabilitationszentren und Abteilungen in Akutkrankenhäusern bis zu Ambulanzen und gemeindenahen Rehabilitationseinrichtungen reicht. Die Akutrehabilitation ist wichtig, um Mechanismen der Plastizität so effektiv und so früh wie möglich zu nützen und das Risiko für Komplikationen zu reduzieren. Dies erfordert nicht nur mobile Teams von Rehabilitationsexperten, die in allen Abteilungen eines Krankenhauses, einschließlich der Intensivstationen, konsilarisch tätig werden, sondern auch speziellen Rehabilitationsbetten - beides unter der Verantwortlichkeit eines Facharztes für PRM. Viele Patienten brauchen auch Rehabilitationsmaßnahmen in eigenständigen Rehabilitationseinrichtungen unter der Leitung von PRMFachärzten. Patienten mit lang dauernden, oft progressiven Behinderungen und Dysfunktionen benötigen eine gemeindenahe rehabilitative Betreuung, um zu gewährleisten, dass ihre Fitness, ihre Gesundheit und ihre Fähigkeiten erhalten bleiben und ihre Unabhängigkeit gefördert wird.

\section{Das Fachgebiet der Physikalischen und Rehabilitative Medizin}

Die Aufgabe der PRM-Fachärzte, die behandelten Krankheitsbilder, die diagnostischen Methoden und Assessments sowie die häufig zur Anwendung kommenden Interventionen werden detailliert in Kapitel 5 und in Anhang 3 dargestellt.

\section{Qualitätsstandards in der Physikalischen und Rehabilitativen Medizin}

1. Die PRM ist in allen europäischen Ländern mit Ausnahme von Dänemark und Malta als unabhängiges medizinisches Fachgebiet vertreten. Die Ausbildung dauert in der Regel 4-6 Jahre. Obwohl es in Europa Unterschiede in der Ausbildung und in den Inhalten des Fachgebietes gibt, hat das European Board for PRM ein umfassendes System der Weiterbildung entwickelt, das ein Curriculum, einen Weiterbildungskatalog und Prüfungen umfasst. Außerdem werden sowohl die Ausbildner als auch die Ausbildungsstätten zertifiziert. Es besteht eine kontinuierliche medizinische Weiterbildung mit dem Ziel einer Rezertifizierung in Abständen von zehn Jahren. Details sind auf der Webseite des Boards abrufbar: www.euro-prm. org. PRM-Fachärzte sind als Dozenten auch in der Ausbildung von Medizinstudenten tätig, zumal die Grundlagen der Rehabilitation allen angehenden Medizinern vertraut sein sollten um eine bessere Versorgung aller Menschen mit Behinderungen sicherzustellen.

2. PRM-Fachärzte begegnen Menschen mit akuten und chronischen Störungen mit einem ganzheitlichen Ansatz. Ihre Hauptaufgabe besteht im Management der Rehabilitation bei bestimmten Krankheiten oder Verletzungen, wie beispielsweise Erkrankungen des Bewegungsapparates, neurologischen Erkrankungen, Traumata, Amputationen, Dysfunktionen der Beckenorgane, kardiorespiratorischer Insuffizienz und Behinderungen aufgrund chronischer Schmerzen und Krebserkrankungen. 
3. PRM-Fachärzte haben die folgenden Kernkompetenzen:

- Medizinische Untersuchungen zur Diagnostik der zugrunde liegenden Krankheit,

- Beurteilung der Funktionsfähigkeit und des Veränderungspotenzials,

- Assessment von Aktivität und Teilhabe sowie von Kontextfaktoren,

- Erstellung eines Rehabilitationsplans,

- Kenntnisse und Erfahrungen in der Anwendung medikamentöser und physikalischer Therapien,

- Evaluation und Quantifizierung der Behandlungsergebnisse,

- Vorbeugung und Behandlung von Komplikationen,

- Prognostizierung von Krankheiten/Störungen und Rehabilitationsergebnissen,

- Kenntnisse von Rehabilitationstechnologien,

- Umgang mit Teamdynamik und Führungsqualitäten,

- Fertigkeiten als Lehrer und Ausbilder,

- Kenntnisse des Sozial- und Gesundheitssystems und der Behindertengesetzgebung.

PRM-Fachärzte treten für die Ausbildung von Medizinstudenten in den Grundsätzen der Rehabilitation ein. Alle Studentinnen und Studenten der Medizin sollten entsprechende Kenntnisse erwerben, um allen Menschen mit Behinderungen eine bessere Betreuung zu ermöglichen.

PRM-Fachärzte sind auch an der Planung und Umsetzung von Rehabilitationsprogrammen beteiligt und fördern die Verbreitung rehabilitativer Grundsätze im Sinne ihrer Patienten. All diese Aktivitäten richten sich an den Bedürfnissen der Bevölkerung aus und zielen auf einen hohen klinischen Standard der erbrachten Leistungen. Regelmäßige Qualitätskontrollen und regelmäßiges Feedback von den Leistungsempfängern sind unabdingbar.

\section{Forschung in PRM}

Die Physikalischen und Rehabilitative Medizin unterliegt uneingeschränkt den Grundsätzen einer wissenschaftlich begründeten (evidenzbasierten) Medizin und fördert ein aktives
Forschungsprogramm, das darauf abzielt, die grundlegenden Prozesse der Rehabilitation zu verstehen und die Determinanten zu erkennen, die für die Genesung und die menschliche Fähigkeit, neue Fertigkeiten zu erwerben und zu lernen, von Bedeutung sind. Der weitere Zuwachs evidenzbasierter Erkenntnisse auf diesem Gebiet hängt allerdings auch von einer besseren finanziellen Unterstützung der einschlägigen Forschung ab.

\section{Zukünftige Entwicklungen in der PRM}

1. Zu den zukünftigen Zielen des Fachgebiets gehört die Entwicklung einer „Kultur der Rehabilitation“, die ein Grundrecht aller Menschen mit Behinderungen darstellt. Die Realisierung dieses Ziels ist eine der wesentlichen Aufgaben von PRM-Fachärzten. Sie ist jedoch nur möglich, wenn umfassende Rehabilitationsmöglichkeiten zur Verfügung stehen. PRMFachärzte werden eine zentrale Rolle bei deren Aufbau übernehmen, mit dem Ziel einen gleicberechtigten und einheitlichen Zugang zur Rehabilitation für alle Menschen in Europa zu gewährleisten. Das Fachgebiet der PRM ist aufs Beste gerüstet, ausgezeichnete klinische Standards durch wissenschaftlich begründete Praxis und die Nutzung modernster Forschungstechnologien zu gewährleisten. Der hohe Nutzen der Physikalischen und Rehabilitativen Medizin wurde durch wissenschaftliche Forschung nachgewiesen und ist Bestandteil des rasch wachsenden medizinischen Wissensstands. Die Aufgabe dieses Buches besteht darin, das Bewusstsein für den hohen Nutzen der Rehabilitation und auch für den positiven Beitrag, den die PRM zu den Lebensbedingungen von Menschen mit Behinderungen leistet, zu verdeutlichen.

2. Eines der Hauptziele des Fachgebiets ist es, den Menschen in allen europäischen Ländern Zugang zur gesamten Breite erstklassiger Rehabilitationsleistungen zu bieten. Die Vertreter des Fachgebiets erachten dies als grundlegendes Menschenrecht. Es ist zu hoffen, dass dieses Buch seinen Lesern ausreichend Informationen an die Hand gibt, um sich gemeinsam mit den PRM-Fachärzten für dieses Ziel zu engagieren. 
1.1 Dieses Buch beschreibt das Wesen, das Arbeitsgebiet und die Charakteristika der Physikalischen und Rehabilitativen Medizin (PRM) in Europa. Es enthält eine Definition des Fachgebiets und erläutert die Kompetenzen der Fachärzte in diesem Gebiet. Es beschreibt den klinischen Kontext der Facharzttätigkeit sowie die Inhalte der Facharztaus- bzw. -weiterbildung. Dieses Buch baut auf dem ersten Europäischen Weißbuch auf, das im Jahr 1989 erschienen ist [1] und sich für die Entwicklung des Fachgebiets in ganz Europa als wichtig erwiesen hat. Es wurde in viele Sprachen übersetzt. 20 Jahre später ist die Erweiterung der Europäischen Union der ideale Anlass für eine Aktualisierung der Informationen über dieses Fachgebiet in Europa, zumal dessen Bedeutung weiter zunimmt und in Medizin und Technologie ständig große Fortschritte erzielt werden.

1.2 Das Buch richtet sich hauptsächlich an drei Gruppen:

- politische Entscheidungsträger im Gesundheitswesen, in der Rehabilitation und im Zusammenhang mit Behinderung;

- die allgemeine Öffentlichkeit und insbesondere Menschen mit Behinderungen sowie die Vertreter ihrer Organisationen sowie

- Fachleute anderer medizinischer Fachgebiete und Gesundheitsberufe.

1.3 Die Medizin in Europa hat durch die Erweiterung der Europäischen Union positive Impulse erhalten, die neue Chancen und Herausforderungen mit sich bringen. Mit dieser Publikation soll der Prozess der Harmonisierung auf dem Gebiet der Physikalischen und Rehabilitativen Medizin zusätzlich gefördert und ein Beitrag dazu geleistet werden, dass Menschen mit Behinderungen im Kontext des Fachgebiets unabhängig von ihrem Wohnort in der erweiterten Gemeinschaft optimal versorgt werden.

1.4 Das Gesundheitswesen ist sowohl auf europäischer Ebene als auch in den einzelnen Ländern großen Veränderungen unterworfen. Die Erwartungen der Öffentlichkeit an die Leistungen der medizinischen Versorgung steigen kontinuierlich an, was die gesellschaftliche Debatte über Menschenrechte und die Verantwortung in der Gesellschaft, vor allem im Hinblick auf die uneingeschränkte Teilhabe von Menschen mit Behinderungen widerspiegelt. Die medizinische Versorgung ist einer kontinuierlichen Weiterentwicklung unterworfen, die klinischen Standards steigen ebenso wie die Nachfrage nach Höchstleistungen, die durch permanente berufliche Weiterentwicklung, eine regelmäßige Qualitätskontrolle und die Fortentwicklung der Facharztausbildung sichergestellt werden kann. Hieraus ergibt sich auch für die Physikalische und Rehabilitative Medizin die Notwendigkeit einer Neudefinition dessen, was sie ist, was sie bieten und wie sie ihr Dienstleistungsangebot und ihr Fachwissen optimal umsetzen kann. Dies beeinflusst auch den Ausbildungsstand, der bei angehenden PRM-Fachärzten vorausgesetzt werden muss. Dieses Weißbuch hat sich die Aufgabe gestellt, diesen Anforderungen zu entsprechen.

\subsection{Rehabilitation}

Rehabilitation wurde von der WHO definiert als „die Anwendung aller Mittel die darauf zielen, die Auswirkungen von Gesundheitsstörungen, die zu Einschränkungen und Behinderungen führen, zu mildern und Menschen mit Behinderungen eine optimale soziale Integration zu ermöglichen“ [2].

Diese Definition schließt die medizinische Rehabilitation ein, unterstützt aber gleichermaßen das Konzept der sozialen Teilhabe, und zwar insofern als das soziale Umfeld an die Bedürfnisse der Menschen mit Behinderungen angepasst werden muss. Ziel ist, soziale Barrieren der Teilhabe unabhängig davon abzubauen, ob diese gesellschaftlicher oder beruflicher Natur sind.

Im Kontext des Gesundheitswesens wurde die Rehabilitation definiert als „ein Prozess der aktiven Veränderung, durch den eine Person, die eine Behinderung erlitten hat, Kenntnisse und Fertigkeiten für eine optimale physische, psychische und soziale Funktion erwirbt“ [3]. Diese Definition verweist stärker auf die aktive Rolle, die Menschen mit Behinderungen bei der Entwicklung ihrer eigenen Fähigkeiten einnehmen. Diese Zielsetzung wird von der medizinischen Rehabilitation wirkungsvoll gefördert.

\subsection{Physikalische und Rehabilitative Medizin}

Die Physikalische und Rehabilitative Medizin in Europa ist ein eigenes medizinisches Fachgebiet und wurde von der UEMS Section of PRM wie folgt definiert:

„Die PRM ist ein unabhängiges medizinisches Fachgebiet, das sich mit der Förderung der körperlichen und kognitiven Funktionsfähigkeit, der Aktivitäten (einschließlich des Verhaltens), der Teilhabe (einschließlich der Lebensqualität) und der Veränderung der persönlichen und der Umweltfaktoren befasst. Sie ist daher für die Prävention, Diagnose, Behandlung und das Rehabilitationsmanagement von Menschen mit zu Behinderungen führenden Gesundheitsstörungen und Komorbiditäten in allen Altersgruppen zuständig.“

PRM-Fachärzte gehen an die Behandlung von Menschen mit akuten und chronischen Gesundheitsstörungen mit einem ganzheitlichen Ansatz heran; Beispiele hierfür sind Erkrankungen des Bewegungsapparates und neurologische Erkrankungen, Amputationen, Dysfunktionen der Beckenorgane, kardiorespiratorische Insuffizienz und Behinderungen infolge chronischer Schmerzen und Krebs.

PRM-Fachärzte arbeiten in unterschiedlichen Einrichtungen. Diese reichen von Akutkrankenhäusern bis hin zu gemeindenahen Rehabilitationseinrichtungen. PRM-Fachärzte wenden spezielle Untersuchungsmethoden an und führen Behandlungen einschließlich pharmakologischer, physikalischer, technischer, edukativer und beruflicher Interventionen durch. Durch ihre umfassende Ausbildung sind sie hervorragend darauf vorbereitet, die Verantwortung für die Arbeit multidisziplinärer Teams zu übernehmen, die Voraussetzung dafür sind, optimale Ergebnisse zu erzielen“ [4]. 
Eine neue umfassende modulare Beschreibung der Physikalischen und Rehabilitativen Medizin auf Grundlage der ICF (s. Kap. 3.2) ist auf internationaler Ebene in der Diskussion (s. Anhang 1a).

Eine Erklärung der beiden Namensbestandteile des Fachgebiets Physikalische und Rehabilitative Medizin findet sich in Anhang $1 b$.

\section{Relevanz der Rehabilitation für Menschen} mit Behinderungen und für die Gesellschaft

\subsection{Epidemiologische Aspekte \\ 3.1.1 Demografische Veränderungen in Europa}

Nach einer britischen Studie $[5,6]$ leiden ungefähr $10 \%$ der westeuropäischen Bevölkerung unter einer Behinderung. Die Lebenserwartung nimmt unter den 700 Millionen Einwohnern Europas - von denen 450 Millionen in den Ländern der Europäischen Union leben - kontinuierlich zu. So ist beispielsweise die Lebenserwartung in Deutschland zwischen 1990 und 2000 um beinahe 3 Jahre angestiegen, und im Jahr 2030 wird einer von vier Einwohnern 65 Jahre oder älter sein $[7,8]$.

Das Älterwerden der Bevölkerungen geht mit einer Zunahme an Behinderungen einher. Dies führt zu höherem Betreuungsaufwand, höheren Kosten für Gesundheit und soziale Versorgung und zu verstärkten Auswirkungen von Komorbiditäten. Zwei Hauptfaktoren bedürfen hier der besonderen Beachtung:

- Eine zunehmende Zahl von Menschen, die eine schwere Krankheit oder ein Trauma überlebt haben, sieht sich komplexen Problemen mit funktionellen Defiziten gegenüber. Viele dieser Menschen sind zum Zeitpunkt des Ereignisses bzw. der Verletzung noch jung und haben noch viele Lebensjahrzehnte vor sich. Hierfür gibt es unzählige Beispiele, wie etwa Schlaganfall, traumatische Hirnverletzung, Polytrauma und Krebs im Kindheitsalter, wo eine bessere Akutbetreuung und Rehabilitation höhere Überlebensraten und bessere Resultate bewirkt haben [9-18].

- Die Ansprüche an einen guten Gesundheitszustand sind in unseren Gesellschaften heutzutage sehr hoch. Damit ist das gesamte Gesundheitssystem - und speziell auch die PRMFachärzte - hohen Erwartungen ausgesetzt.

Der adäquate Umgang mit den Folgen von Krankheiten und Traumata - z. B. Spastizität nach Gehirn- oder Wirbelsäulenverletzungen - bedeutet nicht nur eine Verbesserung der Lebensqualität für den Patienten, sondern bringt auch gesundheitsökonomische Vorteile mit sich, indem die Aufwendungen für die Behandlung von Sekundärkomplikationen geringer werden. Dies hat unmittelbare Folgen auf die Notwendigkeit weiterer Gesundheitsleistungen sowie auf das Arbeitsleben und eventuelle Rentenzahlungen [15-17]. Insbesondere Probleme wie Immobilität, Schmerzen, Ernährung, Inkontinenz, Kommunikationsstörungen, Stimmungs- und Verhaltensstörungen sind neben der Grunderkrankung und Krankheitskomplikationen bedeutsame prädisponierende Faktoren bei der Entstehung von Behinderungen.

Die Rehabilitation reduziert die mit Behinderungen einhergehenden Belastungen effektiver und kann die Chancen und Mög- lichkeiten der Menschen mit Behinderungen wesentlich erhöhen. Es ist nachgewiesen, dass die Rehabilitation oft kostengünstiger ist als ein Weglassen dieser Leistung [14]. Insbesondere bringt die Vermeidung von Komplikationen bei Immobilität (z.B. Dekubitalulzera, Kontrakturen), nach einer Hirnverletzung (z. B. Verhaltensprobleme) und bei Schmerzen (z.B. Stimmungsänderungen) nachweislich viele Vorteile mit sich [19].

\subsubsection{Epidemiologie von Funktionsfähigkeit und Behinderung}

Epidemiologische Studien sind methodologisch traditionell an Krankheitsdiagnosen orientiert. Mittlerweile wird dazu übergegangen, die Komplexität chronischer Krankheiten zu betrachten, bisher allerdings ohne das Konzept der Funktionsfähigkeit, Teilhabe und Lebensqualität von Menschen mit Behinderungen als eigenständiger Bevölkerungsgruppe ernsthaft miteinzubeziehen.

Ein moderner Ansatz zur Lösung dieser Probleme ist z.B. die Fokussierung auf die Konzepte der Healthy Life Expectancy (HALE - Erwartung gesunder Lebenszeit) und der Disability-Adjusted Life-Years (DALYs - Lebensjahre unter Berücksichtigung der durch Behinderung beeinträchtigten Lebenszeit). Es handelt sich dabei um summarische Messungen der Bevölkerungsgesundheit, bei denen Daten über die Sterblichkeit und über nicht lebensbedrohliche Krankheitsergebnisse zu einer einzigen Kennziffer des Gesundheitszustands der Bevölkerung zusammengefasst werden.

Neben der Inzidenz und Prävalenz der häufigsten Krankheiten, die in der Rehabilitation vorkommen (Schlaganfälle, Wirbelsäulenläsionen, traumatische Gehirnverletzungen, Amputationen, rheumatische Erkrankungen, andere neurologische Störungen oder Funktionsstörungen des Bewegungsapparates, chronische Schmerzen usw.) sollte die Epidemiologie in der PRM Folgendes berücksichtigen:

- Verlust der Funktionsfähigkeit im Sinne der ICF-Parameter (vgl. Kap. 3.2);

- natürliche Entwicklung von Funktionen, Aktivitäten und Teilhabe;

- Bedarf an und Zugang zu Ressourcen der Rehabilitation (menschliche Ressourcen, Einrichtungen, Ausrüstungen, Materialien);

- Zugang zu den verfügbaren PRM-Einrichtungen.

Derartige Informationen sind hilfreich bei der Planung und Priorisierung regionaler, nationaler und europäischer Leistungsangebote, bei der Finanzierung der Forschung und bei der Schaffung von Ausbildungskapazitäten. Sie ermöglichen auch Rückschlüsse auf die Wirksamkeit und Wirtschaftlichkeit von PRM-Interventionen.

Es gibt ein umfangreiches Datenmaterial über die Inzidenz und Prävalenz der wichtigsten in der Praxis der Physikalischen und Rehabilitativen Medizin vorkommenden Behinderungen. Einige Beispiele sind in Anhang 3 angeführt. Für die PRM sind insbesondere die funktionellen Auswirkungen von Krankheiten von Interesse, und nach einer neueren Studie aus Portugal sind 0,7\% der Gesamtbevölkerung bettlägerig; $0,4 \%$ sind auf das Sitzen beschränkt (d.h. sie benötigen einen Rollstuhl); 1,9\% leben nicht bei sich zu Hause; 9\% können nicht gehen oder sind erheblich gehbehindert; 8,5\% können sich nur beschränkt ins und aus 
dem Bett bewegen; 6,2\% können die Toilette nicht ohne fremde Hilfe benutzen; 8,6\% benötigen Hilfe beim An- und Auskleiden; 3,6\% der Männer und 5,3\% der Frauen leiden unter einer Harninkontinenz; ca. 2,3\% haben Sprechschwierigkeiten. Die Gesamtprävalenz aller Behinderungen in der Bevölkerung beträgt etwa $10 \%[2]$.

Zusammenfassend ist festzuhalten, dass die epidemiologischen Daten einen hohen Bedarf an Rehabilitation in Europa belegen. Das Fachgebiet der Physikalischen und Rehabilitativen Medizin leistet folglich einen bedeutsamen Beitrag zur Reduktion der Krankheitslast und zur Förderung der Fähigkeiten von Menschen mit Behinderungen.

\subsection{Das WHO-Modell der Funktionsfähigkeit, Behinderung und Gesundheit in der Rehabilitation}

Die WHO veröffentlichte ihre Internationale Klassifikation der Funktionsfähigkeit, Behinderung und Gesundheit im Jahr 2001 [20]. Diese bildete die Grundlage für eine bessere Beschreibung und Analyse der Folgen von Krankheiten und für die Praxis der Physikalischen und Rehabilitativen Medizin.

Die Bewertung der Auswirkungen einer Krankheit auf einen Menschen unterscheidet sich, je nach dem, ob sie vom medizinischen oder vom Rehabilitationsstandpunkt aus vorgenommen wird. Aus der ärztlich-medizinischen Perspektive bzw. aus der Perspektive der Krankheit sind Funktionsfähigkeit, Behinderung und Gesundheit in erster Linie die Folgen oder Auswirkungen einer Erkrankung oder einer gesundheitlichen Störung. Medizinische Interventionen sind auf den Krankheitsprozess gerichtet, und das Endziel dieser Interventionen ist die Vermeidung von Folgen für den Betroffenen oder die Betroffene. Zur Beurteilung der für den Patienten relevanten Ergebnisse einer Intervention müssen sowohl die Gesundheit als auch die Funktionsfähigkeit abgeschätzt werden [21].

\section{Gesundheitsproblem}

(Störung oder Erkrankung)

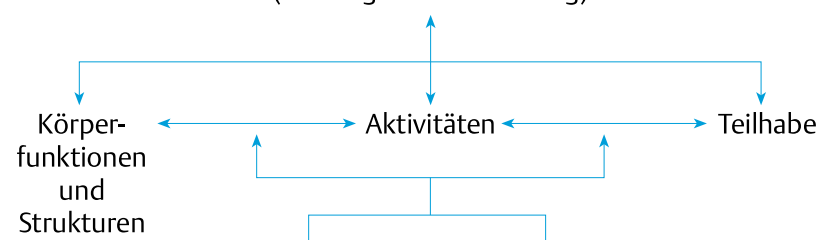

Strukturen

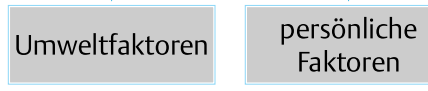

Abb. 1 Das gebräuchliche System von Funktionsfähigkeit und Behinderung - die Internationale Klassifikation der WHO für Funktionsfähigkeit, Behinderung und Gesundheit (ICF) [20].

Aus der PRM-Perspektive betrachtet, sind die Funktionsfähigkeit und Gesundheit der Patienten untrennbar verbunden mit einer gesundheitlichen Störung oder einer Erkrankung, und nicht bloß eine Folge derselben. Außerdem bedeutet die Funktionsfähigkeit nicht nur ein Ergebnis, sondern gleichzeitig den Ausgangspunkt der klinischen Beurteilung und Intervention. Sie ist zudem wichtig für das Qualitätsmanagement, und es ist des weiteren zu beachten, dass die Funktionsfähigkeit in starker Wech- selwirkung mit den individuellen Eigenschaften der Person und mit deren Umwelt steht [22]. Der Rehabilitationsprozess zielt folglich sowohl auf die Funktionsfähigkeit als auch auf die Umwelt und modifizierbare persönliche Faktoren [11]. Die Rehabilitation basiert auf einem tief greifenden Verständnis der Determinanten der Funktionsfähigkeit und von deren Wechselwirkung mit persönlichen und Umweltfaktoren, unabhängig von der jeweiligen Erkrankung.

Für das Verständnis dieser Zusammenhänge und ihre praktische Umsetzung ist das bio-psycho-soziale Modell von Funktionsfähigkeit, Behinderung und Gesundheit der Weltgesundheitsorganisation (WHO) eine gute Grundlage [20]. Die Komponenten dieses Modells $[13,14]$ und ihre Interaktionen sind in Abb.1 dargestellt. Demnach wird die Funktionsfähigkeit mit ihren Komponenten Körperfunktionen und Strukturen, Aktivitäten und Teilhabe im Zusammenhang mit der bestehenden gesundheitlichen Störung sowie $\mathrm{zu}$ persönlichen und Umweltfaktoren betrachtet (Abb.1) [25,26]. Für die Beschreibung der Wechselwirkung zwischen einem kranken Menschen und seinen Kontextfaktoren (Umwelt- und persönlichen Faktoren) ist der Begriff der „Funktionsfähigkeit“ positiver als der der „Behinderung“. Behinderung wird oft als übergeordneter Begriff für Beeinträchtigungen, Aktivitätsbeschränkungen und Einschränkungen in der Teilhabe am gesellschaftlichen/beruflichen Leben verwendet. Dies sollte bei der Durchsicht der einschlägigen Literatur berücksichtigt werden. Von der hier vorgestellten bio-psycho-sozialen Perspektive aus gesehen, geht es bei der Untersuchung von Behinderungen immer auch um die Funktionsfähigkeit im umfassenden Sinne und umgekehrt (Beispiel s. Abb. 2).

- Der Ausdruck „Gesundheitsproblem“ bzw. „Gesundheitsstörung“ bezeichnet übergreifend Krankheiten, Störungen, Verletzungen oder Traumata; er kann zudem noch andere Umstände einschließen, wie Alterung, Stress, angeborene Anomalien oder genetische Prädispositionen. Er kann auch Informationen über Pathogenesen und/oder Ätiologien enthalten. Es bestehen mögliche Wechselwirkungen mit allen Komponenten von Funktionsfähigkeit, Körperfunktionen und Strukturen, Aktivität und Teilhabe.

- Die Körperfunktionen werden definiert als die physiologischen Funktionen der Körpersysteme, einschließlich mentaler, kognitiver und psychologischer Funktionen. Unter den Körperstrukturen sind die anatomischen Bestandteile des Körpers zu verstehen, wie Organe, Glieder und deren Komponenten. Abnormalitäten von Funktion oder Struktur werden als Beeinträchtigungen („Impairments“) bezeichnet, die als signifikante Abweichungen und Verluste (z.B. Deformationen) von Strukturen (z.B. Gelenke) und/oder Funktionen (z.B. reduzierter Bewegungsumfang, Muskelschwäche, Schmerzen und Erschöpfung) definiert sind.

- Unter Aktivität ist die Ausführung einer Aufgabe oder Handlung durch eine Person zu verstehen; sie steht für die individuelle Perspektive der Funktionsfähigkeit.

- Partizipation oder „Teilhabe“ bezieht sich auf die Beteiligung eines Menschen an einer Lebenssituation und steht für die gesellschaftliche Perspektive der Funktionsfähigkeit. Schwierigkeiten auf Ebene der Aktivität werden als Aktivitätsbeeinträchtigung bezeichnet (z.B. Beeinträchtigung der Mobilität, etwas des Gehens, Stiegensteigens, Greifens oder Festhaltens). Probleme, die eine Person mit ihrer Beteiligung an 


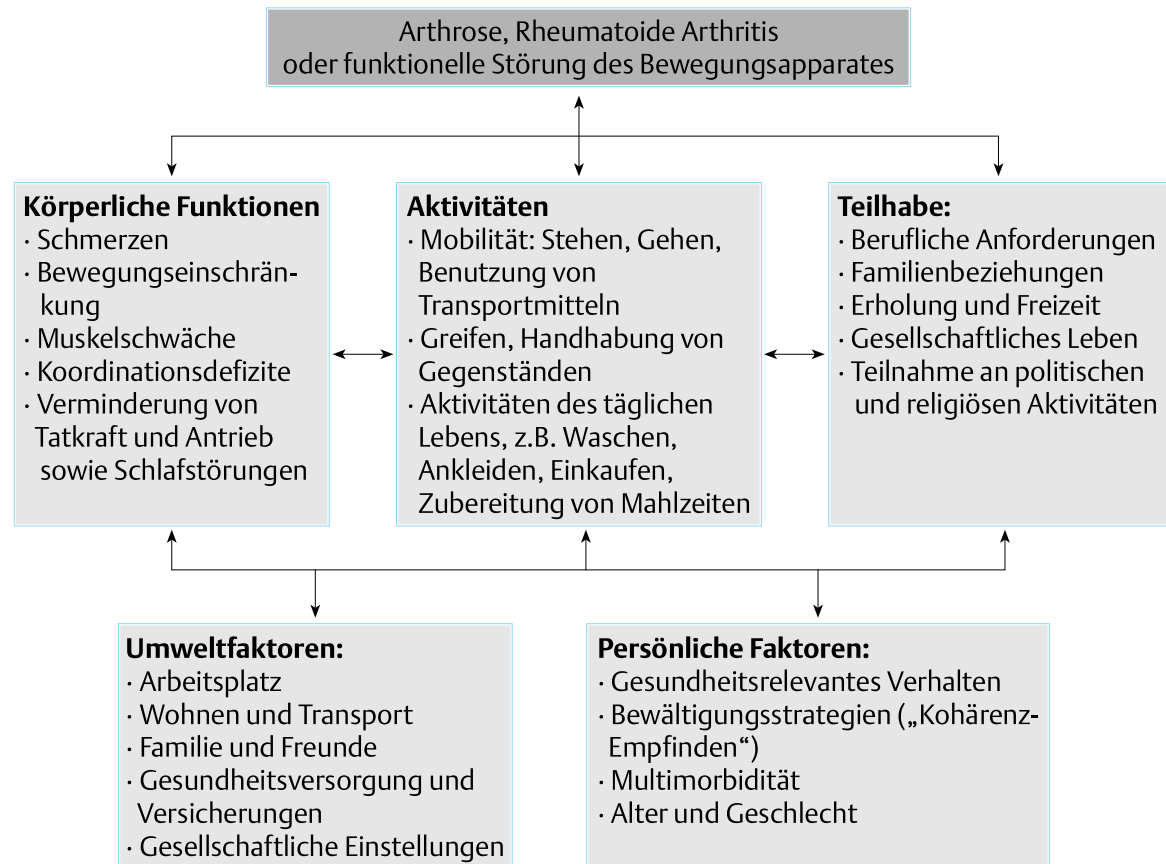

Abb. 2 Beispiel für die Anwendung des ICF-Modells bei Erkrankungen des Bewegungsapparates.
Lebenssituationen hat, werden als Beeinträchtigung der Teilhabe bezeichnet (z. B. Beeinträchtigungen des Lebens in der Gemeinschaft, bei der Erholung und in der Freizeit, kann sich aber beispielsweise auch auf das Gehen beziehen, wenn dieses einen Aspekt der Teilhabe im Hinblick auf Lebenssituationen darstellt).

- Die Umweltfaktoren stellen den kompletten Hintergrund des Lebens und der Lebenssituation eines Menschen dar. Im Rahmen der Kontextfaktoren bilden die Umweltfaktoren das physische, gesellschaftliche und einstellungsbezogene Umfeld, in dem sich die Menschen befinden und ihr Leben gestalten. Diese Faktoren liegen außerhalb der Person und können diese positiv oder negativ beeinflussen, d. h. sie können für den Einzelnen einen Förderfaktor oder eine Barriere darstellen.

- Zu den persönlichen Faktoren gehören der individuelle Lebenshintergrund und die Lebenssituation eines Menschen; sie erstrecken sich auch auf Aspekte, die nicht unmittelbar mit der Erkrankung zu tun haben, wie Geschlecht, Alter, Rasse, Fitness, Lebensstil, Gewohnheiten und gesellschaftlicher Hintergrund. Die Risikofaktoren könnten demnach in persönliche (z.B. Lebensstil, genetischer Aufbau) und Umweltfaktoren (z. B. bauliche Hindernisse, Lebens- und Arbeitsbedingungen) unterteilt werden. Die Risikofaktoren werden nicht nur in der Anfangsphase einer Erkrankung wirksam, sie interagieren vielmehr in jeder Phase des Krankheitsverlaufs mit dem Behinderungsprozess.

Die Physikalische und Rehabilitative Medizin fördert die Funktionsfähigkeit einer Person mit einer multidisziplinären Strategie $[27,28]$. Voraussetzung für den Erfolg sind die fundierte Beurteilung und das umfassende Verständnis der Funktionsfähigkeit der betroffenen Person.

Für unterschiedliche Erkrankungen wurden auf internationaler Ebene ICF-Core-Sets entwickelt [29-31]. Sie sollen die praktische Anwendung der ICF erleichtern und enthalten so wenige ICF-Domänen wie möglich und so viele wie nötig, um ein typi- sches Problemspektrum bezüglich der Funktionsfähigkeit von Patienten mit bestimmten Erkrankungen erschöpfend zu beschreiben. Sie dienen zur umfassenden, multidisziplinären Bewertung von Behinderungen und kommen in klinischen Studien zur Anwendung. Zur Verbesserung der internen Teamkommunikation und der Dokumentation in der multidisziplinären Versorgung kann ein ICF-Bewertungsbogen in Kombination mit den ICF-Core-Sets verwendet werden $[32,33]$.

\subsection{Ethische Aspekte und Menschenrechte 3.3.1 Behinderung und Menschenrechte}

In der Vergangenheit wurde es als ausreichend erachtet, Menschen mit Behinderungen medizinisch und pflegerisch zu versorgen. In den letzten 20 Jahren hat in Europa ein grundlegender Wandel der Einstellung stattgefunden, demzufolge Menschen mit Behinderungen als Staatsbürger mit voller Autonomie und uneingeschränkten Menschenrechten zu betrachten sind. Diese Haltung drückt sich im Geist der UN-Menschenrechtsnormen aus und hat allenthalben zu Gesetzen geführt, die jede Diskriminierung aufgrund von Behinderungen verhindern sollen.

Die Menschenrechtserklärung der UN [35] besagt, dass ein Mensch mit einer Behinderung nicht sein gesamtes Leben lang ein „Betreuter“ (ein „Patient“) bleiben darf. Er oder sie ist ein(e) Staatsbürger(in) mit besonderen Bedürfnissen infolge seiner speziellen Behinderung. Diese Bedürfnisse müssen von der Gesellschaft berücksichtigt werden, und das in einem „normalen“ Kontext. Das Prinzip der Teilhabe ist fundamental, und ein zentraler Aspekt davon ist der offene Zugang zur Gesellschaft. Das schließt den physischen Zugang - beispielsweise in öffentliche und private Bereiche bzw. Gebäude - ebenso ein wie die Erreichbarkeit öffentlicher Transportmittel und den Zugang zu Informationen u. a. In einigen europäischen Staaten wurden Gesetze zur behindertengerechten Gestaltung öffentlicher Bauwerke erlassen. Die Generalversammlung der Vereinten Nationen bewilligte im Dezember 1993 die Einführung von UN-Standards, die derzeit zu einem Abkommen weiterentwickelt werden, um Menschen 
mit Behinderungen die volle Teilhabe und Gleichberechtigung zu ermöglichen. Dies ist von Bedeutung für die Festlegung von Handlungsgrundsätzen.

Auch der Europarat hat eine Reihe von Berichten und Dokumenten in den Grundrechten für Menschen mit Behinderungen veröffentlicht. Im Zentrum steht eine Erklärung der in Europa für Integration von Menschen mit Behinderungen zuständigen Minister, die 2003 in Malaga verabscheidet worden ist [36]. In dieser Deklaration sind folgende Ziele formuliert:

- die Lebensqualität von Menschen mit Behinderungen und deren Familien innerhalb eines Jahrzehnts zu verbessern;

- Maßnahmen zu ergreifen, die auf eine Verbesserung der Lebensqualität von Menschen mit Behinderungen abzielen, und die auf einer gründlichen Bewertung von deren Situation, Potenzial und Bedürfnissen beruhen;

- einen Aktionsplan zur Realisierung dieser Ziele zu entwickeln;

- einen gleichberechtigten Zugang zu Beschäftigung als Schlüsselelement der sozialen Teilhabe zu ermöglichen;

- innovative Methoden (der Rehabilitation) zu entwickeln und anzuwenden, die der höheren Lebenserwartung von Menschen mit körperlichen, psychischen und intellektuellen Beeinträchtigungen Rechnung tragen;

- Maßnahmen zur Erhaltung einer guten physischen und mentalen Gesundheit in den späteren Lebensphasen zu ergreifen;

- im Umfeld von Menschen mit Behinderungen, die umfangreicher Hilfe bedürfen, supportive Strukturen aufzubauen;

- eine hohe Qualität der erbrachten (Rehabilitations-)Leistungen sicherzustellen;

- Programme und Ressourcen zur Erfüllung der Bedürfnisse von Menschen mit Behinderungen zu entwickeln.

Im Jahr 2005 hat die Weltgesundheitsversammlung eine Resolution über „Behinderung, einschließlich Prävention, Management und Rehabilitation“ angenommen (World Health Assembly Resolution 58.23). Darin wurden den Mitgliedsstaaten unter anderem folgende Verpflichtungen aufgetragen:

- Teilnahme an Aktivitäten zur Behinderungsprävention;

- Förderung und Unterstützung von Rehabilitationsprogrammen auf kommunaler Ebene in Verbindung mit der primären Gesundheitsversorgung und ihrer Integration in die Gesundheitssysteme;

- Ermöglichung des Zugangs zu adäquaten unterstützenden Technologien und Förderung von deren Entwicklung und der Entwicklung anderer Hilfsmittel, welche der Eingliederung von Menschen mit Behinderungen in die Gesellschaft dienlich sind;

- Erforschung und Anwendung der wirksamsten Maßnahmen zur Verhinderung von Behinderungen sowie deren Umsetzung in die Praxis;

- Bereitstellung adäquater und wirksamer medizinischer Versorgung für Menschen mit besonderen Bedürfnissen sowie des Zugangs zu einer solchen Versorgung, einschließlich Prothesen, Rollstühle, Fahrhilfen und anderer Hilfsmittel;

- Erforschung und Implementierung der wirksamsten Maßnahmen zur Verhinderung von Behinderungen in Zusammenarbeit mit Gemeinden und anderen Sektoren.
Zudem wurde der Generaldirektor damit beauftragt,

- die Zusammenarbeit innerhalb der Organisation im Sinne einer Steigerung der Lebensqualität und Förderung der Rechte und Würde von Menschen mit Behinderungen zu intensivieren;

- die Mitgliedsstaaten beim Ausbau ihrer nationalen Rehabilitationsprogramme zu unterstützen;

- die Mitgliedsstaaten beim Sammeln zuverlässiger Daten über alle relevanten Aspekte zu unterstützen, etwa über die Wirtschaftlichkeit von Interventionen zur Prävention von Behinderungen, zur Rehabilitation und Pflege;

- die Zusammenarbeit innerhalb der Vereinten Nationen und zu den Mitgliedsstaaten, den NGOs (nichtstaatlicher Organisationen) und Organisationen von Menschen mit Behinderungen weiter zu stärken;

- Studien über die Inzidenz und Prävalenz von Behinderungen als Grundlage für die Formulierung von Strategien zur Prävention, Behandlung und Rehabilitation zu fördern.

In verschiedenen europäischen Ländern sind die Rechte der Menschen mit Behinderungen gesetzlich verankert [37]. In den Rechtssystemen mancher Länder gibt es bereits eine längere Tradition hinsichtlich einer allgemeinen Politik zur Rehabilitation von Menschen mit Behinderungen (z.B. ist in Frankreich seit 1975 ein Gesetz über die Rechte von Menschen mit Behinderungen in Kraft). In den meisten Staaten wurden entsprechende Gleichbehandlungsgesetze allerdings erst in den letzten 15 Jahren geschaffen, z. B. das Gleichbehandlungsgesetz für Behinderte (Deutschland), das Rahmengesetz (Italien), das Verfassungsgesetz (Finnland), das Gesetz über die Rechte von Personen mit Behinderungen (Ungarn 1998), Gesundheit für Alle (Slowenien 2004), das Behinderten-Gleichbehandlungsgesetz (UK 1996), das Gesetz für Eingliederung (UK 2001) usw. All diese Gesetze, Regelungen und Grundsatzerklärungen haben in die PRM-Praxis Eingang gefunden und werden von PRM-Fachärzten unterstützt.

\subsubsection{Fragen zu Geschlecht, Rasse, Kultur, Religion und sexueller Orientierung}

Der gleichberechtigte Zugang zur Rehabilitation und sozialen Teilhabe ohne jede wie auch immer geartete Diskriminierung ist ein unverzichtbares Grundprinzip für die Bereitstellung und praktische Anwendung von Rehabilitationsmaßnahmen. Die PRM-Fachärzte sind sich der Belastungen bewusst, denen vielen Menschen aufgrund von Unterschieden in Geschlecht, Rasse, Religion und sexueller Orientierung ausgesetzt sind. Durch Schwächung von Körperselbstbild, psychologischem Zustand und Wohlbefinden kann gesellschaftliche Diskriminierung die Anpassungs- und Integrationsfähigkeit von Menschen mit Behinderungen beeinträchtigen. Die Rehabilitation verfolgt einen ganzheitlichen Ansatz, um die unabhängige Lebensgestaltung der Menschen zu unterstützen, und für viele stellen Religion und Spiritualität wichtige Stützen im Rehabilitationsprozess dar.

In den Rehabilitationseinrichtungen gibt es bereits etliche Programme zur Förderung eines gleichberechtigten Zugangs für alle, z.B. um Müttern die Teilnahme an Rehabilitationsprogrammen zu erleichtern.

\subsubsection{Das Recht auf Rehabilitation}

Der Zugang zur Rehabilitation ist ein grundlegendes Menschenrecht. Die europäische Gesetzgebung stellt klar, dass Menschen 
mit Behinderungen einen Zugang zu adäquater Rehabilitation haben müssen. Es ist deshalb auch erforderlich, dass alle mit Rehabilitation befassten Fachkräfte - einschließlich PRM-Fachärzte - gut ausgebildet sind und die notwendigen Fertigkeiten besitzen. Die Physikalische und Rehabilitative Medizin spielt in diesem Versorgungssystem eine bedeutende Rolle, nicht zuletzt in der Beratung über die Entwicklung von Dienstleistungen und in der Zusammenarbeit mit staatlichen Behörden und nichtstaatlichen Organisationen. Die PRM unterstützt auch Organisationen von und für Menschen mit Behinderungen mit dem Ziel, einen Zugang zu Rehabilitation und gesellschaftlicher Integration $\mathrm{zu}$ schaffen. Dies ist ein wichtiges Ziel, zumal die Verwirklichung der vollständigen Menschenrechte und die Verhinderung von Diskriminierung weitere Aktivitäten und Entwicklungen erfordern. Dies ist auch eine wesentliche Aufgabe der PRM-Sektion der Union Européenne des Médecins Spécialistes (UEMS) (vgl. Kapitel 6.4).

Der Europarat hat eine Gesetzgebung zu Behindertenrechten vorgeschlagen, die im Wesentlichen folgende Bereiche umfasst [37,38]:

- Behinderungsprävention und Gesundheitsaufklärung;

- Identifizierung und Diagnose;

- Behandlung und therapeutische Hilfsmittel;

- Ausbildung;

- berufliche Anleitung und Weiterbildung;

- Beschäftigung;

- gesellschaftliche Integration und Umwelt;

- gesellschaftlicher, wirtschaftlicher und rechtlicher Schutz;

- Aus- und Weiterbildung für Menschen, die mit der Rehabilitation und sozialen Integration von Menschen mit Behinderungen zu tun haben;

- Information;

- Statistik und Forschung.

In Regel 3 des UN-Standards [39] wird festgehalten, dass die „Regierungen ihre eigenen Rehabilitationsprogramme für alle Gruppen von Personen mit Behinderungen entwickeln“ sollten. Solche Programme müssen auf den Bedürfnissen der Menschen mit Behinderungen und auf den Grundlagen der vollen Teilhabe und Gleichheit aufbauen. All jene, die der Rehabilitation bedürfen, sollten Zugang dazu haben. Dies gilt auch für Menschen mit ausgeprägten oder multiplen Behinderungen. Die Regierungen sollten bei der Entwicklung und Evaluierung solcher Rehabilitationsprogramme das Know-how der Organisationen für Menschen mit Behinderungen nützen. Leider wird dieser Standard in den meisten europäischen Ländern nur zum Teil eingehalten, was für PRM-Ärzte ebenso wie für die Gesellschaft im Ganzen eine Herausforderung darstellt. Die PRM-Praxis basiert - wie erwähnt - nicht auf einem ausschließlich medizinischen Modell, sondern berücksichtigt auch soziale Aspekte und nimmt eine ganzheitliche Betrachtungsweise ein. Sie gründet sich auf ein biopsychosoziales Modell, das die Versorgungsgrenzen überschreitet und in dem sowohl persönliche als auch Umweltfaktoren einbezogen sind. Sie bietet den Betroffenen ein persönliches „Empowerment“, das sie in ihrer vollen Teilhabe an allen Lebensbereichen unterstützt.

Trotz der Verankerung als Menschenrecht im Rahmen des Internationalen Jahres für behinderte Menschen (1981) und des Europäischen Jahres für Menschen mit Behinderungen (2003) bleibt der Zugang zur Rehabilitation nach Verletzung oder Krankheit nach wie vor problematisch. Dies liegt zum Teil an einem Mangel an Ressourcen, an Informationen für Menschen mit Behinderungen und an der mangelhaften Organisation der Dienstleistungen, woraus ein Missverhältnis zwischen Angebot und Bedarf entsteht. Die Teilhabe vieler Menschen mit Behinderungen wird auch von traditionellen Einstellungen in der Gesellschaft behindert. Umso größeres Augenmerk muss auf die weitere Verbesserung der Gesundheit und Aufklärung der Menschen mit Behinderungen gelegt werden, um diese noch besser in das gesellschaftliche Leben einzugliedern. Der Physikalischen und Rehabilitativen Medizin kommt in diesem Prozess eine bedeutende Rolle zu.

\subsubsection{Ethische Fragen einer gesundheitsbezogenen Rehabilitation}

Menschen mit Behinderungen sollten an der Einrichtung und Fortentwicklung von Rehabilitationsdiensten aktiv beteiligt werden. Ein gutes Beispiel für ihre Einbindung in ein Rehabilitations-Teamwork war das EU-HELIOS-Programm (1990-1996), dessen Ziel es war, die Chancen von „behinderten Menschen in Europa auf ein unabhängiges Leben in einer offenen Gesellschaft“ zu verbessern. Eine der Arbeitsgruppen gab folgende Empfehlungen für eine gute Rehabilitationspraxis:

- Die Person mit Behinderungen muss im Mittelpunkt eines multidisziplinären Ansatzes stehen und sollte in der Lage sein, aufgrund von Informationen eine selbstbestimmte Wahl der Behandlung zu treffen. Er oder sie sollte an dem Prozess in vollem Umfang teilhaben und sein/ihr Recht auf den Empfang von Leistungen unabhängig von der Art der Behinderung, von Alter, Geschlecht, Religion, ethnischer Herkunft, Wohnort und finanziellen Ressourcen durchsetzen können.

- Wenn nötig sollte die Familie mit einbezogen werden.

- Kontinuierliche und koordinierte Maßnahmen sollten eine Rückkehr in die normale Umgebung und in ein selbstbestimmtes gesellschaftliches und berufliches Leben ermöglichen.

- Die Rehabilitationsstrategien müssen einer Evaluation durch die Betroffenen unterzogen werden.

Alle Rehabilitationsabteilungen, Programme und Praktiken sollten unter Berücksichtung ethischer und Menschenrechtsaspekte klare operative Pläne ausarbeiten, um:

- die Menschen jederzeit mit Würde und Respekt zu behandeln;

- erreichbare Informationen bereitzustellen, um den Patienten den Entscheidungsprozess zu erleichtern;

- auf Basis umfassender Informationen das Einverständnis der Betroffenen einzuholen und die Ablehnung des Einverständnisses zu ermöglichen;

- festzustellen, ob Patienten die Fähigkeiten haben, kompetente Entscheidungen zu treffen;

- die Privatsphäre der Patienten zu schützen und den Schutz vertraulicher Patientendaten sicherzustellen;

- physischen oder psychischen Missbrauch zu verbieten;

- Verständnis für kulturelle, religiöse und andere Haltungen und Überzeugungen sowie für andere Behandlungspraktiken zu haben;

- architektonische, Einstellungs-, Kommunikations-, berufliche und andere Barrieren für die betreffenden Personen zu beseitigen. 


\subsection{Rehabilitation und Gesundheitssysteme}

Die Zugangsmodalitäten und die Finanzierung von Rehabilitationsleistungen variieren zwischen den Staaten und sind von den unterschiedlichen Gesundheits- und Gesellschaftssystemen abhängig. Die Akteure innerhalb dieser Systeme sind unter anderem Politiker, Planer und Organisationen, die für die Finanzierung von Gesundheits- und Sozialleistungen zuständig sind; dazu kommen Selbsthilfegruppen und andere engagierte Personen in den verschiedenen Körperschaften.

Nationale und regionale Unterschiede im Zugang zu Rehabilitationsleistungen sind überwiegend durch die verschiedenen Systeme der Finanzierung von akuten und weiterführenden Rehabilitationsmaßnahmen bedingt; in einigen Ländern entscheiden die medizinischen Dienste der Versicherungsanstalten über den Zugang zur Rehabilitation, vor allem im Gebiet der Rehabilitation bei chronischen Erkankungen. In anderen Ländern und in der Akutrehabilitation werden die Patienten von Allgemeinmedizinern und anderen Fachärzten zu den Rehabilitationszentren zugewiesen. Wiederum in anderen Ländern können sich die Patienten direkt an die stationären Abteilungen in Rehabilitationskliniken wenden. In einigen Ländern können praktische Ärzte die Patienten zur fachärztlichen ambulanten Rehabilitationsbehandlung zuweisen; in anderen wird in diesem Bereich auch der selbstbestimmte Zugang durch die Patienten akzeptiert. Eine detaillierte Einzelbeschreibung ist aufgrund der zahlreichen unterschiedlichen Systeme in Europa nicht möglich; die nötigen Informationen sind in den einzelnen Mitgliedsländern erhältlich.

Auch die Struktur der Rehabilitationsdienste variiert in Europa, und die Versorgung ist keineswegs flächendeckend. In vielen Ländern gibt es nur wenige PRM-Fachärzte. Trotz der weit verbreiteten Anerkennung des Fachgebiets in ganz Europa sind pro Land große Unterschiede in der Anzahl der Fachärzte, in deren Funktion im Gesundheitssystem und in ihren Arbeitsbedingungen zu verzeichnen. In Anhang 2 sind die Facharztzahlen nach Ländern aufgelistet. Zwar muss eine optimale Zahl von PRMFachärzten pro Kopf der Bevölkerung in Europa noch ermittelt werden, dennoch sind die großen Unterschieden zwischen den Staaten evident.

\subsection{Ziele und Ergebnisse der Rehabilitation}

Das Rehabilitationspotenzial einer Person (d.h. die Kapazität, von einer Rehabilitation zu profitieren) kann nur in Kenntnis seiner/ihrer Krankheitsgeschichte ermittelt werden. Einige Personen erholen sich spontan, sodass eine frühzeitige Intervention den falschen Eindruck einer erfolgreichen Behandlung erwecken könnte $[40,41]$. Andererseits bedeutet für viele Menschen ein Mangel an Rehabilitationsleistungen eine Schmälerung ihrer Unabhängigkeit und Lebensqualität [42]. Kontrollierte Studien, in denen diese Faktoren berücksichtigt wurden, haben gezeigt, dass eine frühzeitige Intervention mit einem besseren Endergebnis einhergeht, unabhängig davon, ob es zu einer vollständigen Erholung kommt oder nicht. Aber auch eine verzögerte oder späte Intervention kann noch Vorteile erbringen $[43,44]$.

Das Hauptziel der Rehabilitation besteht darin, Menschen mit Behinderungen zu ermöglichen, das von ihnen gewünschte Leben unter Berücksichtigung unvermeidlicher Einschränkungen ihrer Aktivitäten infolge der krankheits- oder verletzungsbe- dingten Beeinträchtigungen zu führen. In der Praxis wird dieses Ziel oft mit einer Kombination von Maßnahmen erreicht:

- Beeinträchtigungen überwinden oder handhaben,

- Barrieren gegen die Teilhabe an dem von der Person gewählten Umfeld abbauen oder verringern und

- ihre Wiedereingliederung in die Gesellschaft unterstützen.

Im Sinne eines patientenzentrierten Prozesses sollen seine Aktivitäten und Teilhabe gefördert werden.

Jeder Rehabilitationsplan muss deshalb die Wünsche und Ressourcen der einzelnen Patienten, die Prognose ihrer behindernden Erkrankung, die Art ihrer körperlichen und kognitiven Beeinträchtigungen und ihre Kapazität zum Erwerb neuer Kenntnisse und Fertigkeiten berücksichtigen, die sie in die Lage versetzen können, ihre Aktivität zu steigern und die Teilhabe zu verbessern. Zudem muss abgeschätzt werden, in welchem Ausmaß die umweltbedingten Barrieren gegen eine Teilhabe (ob aufgrund der physischen Umwelt selbst oder aufgrund des Verhaltens anderer Menschen) abgebaut werden können. Schließlich ist noch zu eruieren, ob die Ressourcen zur Durchführung des Rehabilitationsplans vorhanden sind. Das Wohlbefinden einer Person und ihrer sozialen Teilhabe ist ein wichtiges Zielkriterium für eine patientenzentrierte Rehabilitation [44].

Das individuelle Wohlbefinden der Betroffenen ist wahrscheinlich ein zuverlässigerer Erfolgsindikator als die Lebensqualität, da die in der Rehabilitation verfolgten Ziele den individuellen Wünschen der oder des Einzelnen gerecht werden müssen. $\mathrm{Zu}$ berücksichtigen ist, dass verschiedene Menschen, die sich in vergleichbaren Situationen befinden, sehr wohl unterschiedliche Ziele haben können. Viele heute zur Beurteilung der Lebensqualität verwendeten Messinstrumente enthalten implizit Urteile über die Relevanz bestimmter objektiver Faktoren, etwa der Fähigkeit zum Treppensteigen, die aber nicht von allen Menschen mit Behinderungen als gleich wichtig empfunden werden.

Die Rehabilitation ist in der Lage, die mit einer Behinderung einhergehenden Belastungen für Einzelne und für die Gesellschaft zu reduzieren. Es hat sich gezeigt, dass durch die Zunahme der Aktivität, die Verbesserung des Gesundheitszustandes, die Reduzierung von Krankheitskomplikationen und die Auswirkungen von Begleiterkrankungen im Rahmen der Rehabilitation die individuelle Funktionsfähigkeit und Unabhängigkeit der Betroffenen gesteigert werden kann. Die größere persönliche Autonomie, die verbesserten Chancen im Beruf und bei anderen Aktivitäten sind nicht nur für die unmittelbar Betroffenen ein Gewinn, sondern für die Gesellschaft insgesamt von Nutzen. Angesichts der zahlreichen gesellschaftlichen Faktoren, die für eine Rückkehr in ein unabhängiges Leben und Arbeiten von Bedeutung sind, kommt der PRM die Aufgabe zu, die Betroffenen und ihre Familien/Betreuer(innen) darauf vorzubereiten, die bestehenden Möglichkeiten optimal zu nutzen.

Die Rehabilitation hat sich nicht nur darin als wirksam erwiesen, die individuelle Funktionsfähigkeit und Unabhängigkeit zu fördern, sondern auch die krankheitsbedingten Kosten zu senken [45]. Es wurde gezeigt, dass die für spezielle Rehabilitationsmaßnahmen aufgewendeten Mittel durch nachfolgende Einsparungen bis zum 17 fachen Wert wieder eingebracht werden [46, 47]. 
Um die Wirksamkeit rehabilitativer Interventionen und Dienstleistungen evaluieren zu können, ist es notwendig, die Rehabilitationsergebnisse auf der individuellen Ebene zu messen. Diese Ergebnismessungen müssen unmittelbar mit den im Rehabilitationsplan vorgesehenen, spezifischen Zielen in Beziehung gesetzt werden. Die Evaluation der Rehabilitation unterscheidet sich grundsätzlich von der Evaluation krankheitsorientierter medizinischer Behandlungen, die auf eine Dämpfung der Krankheitsaktivität oder eine Heilung der Krankheit abzielen. Eine Rehabilitation kann auch dann erfolgreich sein, wenn eine biologische Heilung nicht möglich ist, ja sogar bei vorübergehenden oder sich kontinuierlich verschlechterndem Krankheitsbild. Im letzteren Fall muss die Rehabilitation kontinuierlich weitergeführt werden, um die Patienten in die Lage zu versetzen, ein bestimmtes Ausmaß an Teilhabe und Wohlbefinden aufrechtzuerhalten, das andernfalls nicht möglich wäre. Eine Qualitätssicherung von Rehabilitationsmaßnahmen und sozialen Leistungen sollte routinemäßig durchgeführt werden.

\section{Grundlagen der Physikalischen und Rehabilitativen Medizin}

\subsection{Lernprozesse als Grundlage der Physikalischen und Rehabilitativen Medizin}

Lernen ist ein bewährtes und modernes Element des Rehabilitationsprozesses. PRM-Fachärzte sind immer auch Lehrer, insbesondere wenn die Rehabilitationsprogramme von neuen Konzepten der Adaptation (z. B. Plastizität) und motorischem Lernen unterstützt werden müssen. PRM-Fachärzte müssen die Grundlagen von Adaptation und Plastizität kennen und den theoretischen Hintergrund der Grundlagen des Lernens und Lehrens verstehen [48].

Die Kenntnis dieser Prinzipien ist Voraussetzung für die Planung von Strategien, die zur Verbesserung der funktionellen Ergebnissen führen und krankheitsbedingte Fehlanpassungen verhindern können. Effektive moderne Konzepte des motorischen Lernens und der Heilung werden mit dem Ziel entwickelt, den Erwerb von Fertigkeiten zu fördern, die für das Alltagsleben der Patienten relevant sind. Ein derartiger Ansatz trägt dazu bei, erlernte Schonhaltungen zu vermeiden und die normalen Funktionen wiederherzustellen. $\mathrm{Zu}$ intensive Programme können aber ebenfalls schädlich sein [49]. Im Allgemeinen besteht das Lernen aus Anweisungen darüber, wie etwas zu tun ist oder wie eine Aufgabe zu lösen ist. Aber auch ohne explizite Anleitung hat eine Person die Möglichkeit, sich über implizites Lernen neues Verhalten oder neue Fertigkeiten anzueignen.

Explizites und implizites Lernen scheinen über unterschiedliche Nervenbahnen zu verlaufen. Der implizite Lernprozess ist gegenüber neurologischen Schädigungen weniger empfindlich, insbesondere wenn es zu schweren Beeinträchtigungen des Erinnerungsvermögens gekommen ist. Auch wenn der erste Ansatz zur Zeit in der Therapie öfter verwendet wird, so können beide - das explizite wie das implizite Lernverfahren - durch geeignete Techniken der Physikalischen und Rehabilitativen Medizin therapeutisch genutzt werden [50].

\subsection{Ziele der Physikalischen und Rehabilitativen Medizin}

Die Hauptziele der Physikalischen und Rehabilitativen Medizin sind die Optimierung von sozialer Teilhabe und Lebensqualität. Das beinhaltet in der Regel, dass die betroffene Person in die Lage versetzt wird, über Art und Ausmaß der angestrebten Selbstständigkeit und Unabhängigkeit selbst zu entscheiden, und zwar einschließlich der Teilhabe an beruflichen, gesellschaftlichen und Freizeitaktivitäten und im Einklang mit ihren Grundrechten [50].

Die Physikalische und Rehabilitative Medizin ist auf fünf Arten wirksam:

- Behandlung der zugrunde liegenden Krankheit;

- Reduzierung der Funktionsstörung und/oder Behinderung;

- Prävention und Behandlung von Komplikationen;

- Verbesserung von Funktionsfähigkeit und Aktivität;

- Ermöglichung der Teilhabe.

Bei allen Interventionen der PRM wird nach Maßgabe der ICF der persönliche, kulturelle und umweltbezogene Kontext der Betroffenen berücksichtigt (vgl. Kapitel 3.2).

Die Rehabilitation ist ein kontinuierlicher und koordinierter Prozess, der mit dem Beginn einer Krankheit oder zum Zeitpunkt der Verletzung einsetzt und so lange fortgesetzt wird, bis die betroffene Person Stellung und Funktion in ihrem sozialen Umfeld erreicht hat, die ihren lebenslangen Bestrebungen und Wünschen entspricht.

\subsection{Das Rehabilitationsteam \\ 4.3.1 Teamwork in der Rehabilitation}

Rehabilitation ist eine multidisziplinäre Aufgabe [4], die auf einer guten Kommunikation im Team und den individuellen Fertigkeiten der beteiligten Fachkräfte beruht. Damit die Rehabilitation funktioniert, muss das Team gemeinsam mit den Patienten klare Rehabilitationsziele definieren, an deren Formulierung dem Patienten nahe stehende Personen mitwirken sollten. Die Bedeutung der Teamarbeit besteht in diesem Zusammenhang darin, dass das Ergebnis des Teams mehr ist als die Summe der einzelnen fachlichen Inputs. Das Team selbst profitiert durch gemeinsames Know-how und die Verteilung der Arbeitslast auf die Teammitglieder. Zwischen den Aufgaben der einzelnen Teammitglieder bestehen keine starren Abgrenzungen, und erfolgreiche Teams leben von den Beiträgen aller Mitglieder, unabhängig von beruflichen Grenzziehungen. Die meisten Rehabilitationsteams für Menschen mit einem speziellen Rehabilitationsbedarf bestehen aus einem Kernteam von verschiedenen Berufsgruppen mit unterschiedlichen Spezialisierungen.

\subsubsection{Multidisziplinäre Rehabilitationsteams}

Die spezialisierten medizinischen Rehabilitationsteams werden von PRM-Fachärzten geleitet. Das Team ist mehr als eine Ansammlung verschiedener Berufsgruppen, vielmehr arbeiten die verschiedenen Teammitglieder in gegenseitiger Kenntnis und Anerkennung der Fähigkeit des anderen zusammen. Das Team arbeitet darüber hinaus mit den Patienten und ihren Familien zusammen, um angemessene, realistische und zeitlich sinnvolle Ziele in einem umfassend koordinierten Rehabilitationsprogramm zu erreichen. Diese Ziele werden im Lauf der Zeit an die erzielten Therapiefortschritte angepasst. Sie sind patientenzentriert und basieren auf der gemeinsamen Empfehlung aller in den 
Rehabilitationsprozess involvierten Berufsgruppen. Die Frage des Teams sollte nicht lauten, „Welche Ziele hat die Ergotherapeutin diese Woche?“, sondern „Welche Ziele hat der Patient diese Woche und wie kann die Ergotherapeutin dazu beitragen?“ Auf diese Weise vermag die Rehabilitation die Funktionsfähigkeit und Teilhabe der Patienten durch Bereitstellung einer koordinierten Quelle für Informationen, Beratung und Behandlung des Menschen mit Behinderungen und seiner/ihrer Familie zu verbessern, wobei das Team als Leistungserbringer und als Katalysator fungiert.

Die Zusammenarbeit innerhalb des Rehabilitationsteams ist durch eine strukturierte Teamkommunikation und regelmäßige Teamkonferenzen gewährleistet. Die Diagnose, die Auswirkungen auf Funktionsfähigkeit und Aktivitäten sowie die Fähigkeit der Patienten zur Teilhabe an der Gesellschaft werden systematisch diskutiert. Gleichzeitig erfolgt ein Austausch über die möglichen Risiken und die Prognose der Krankheit. Kurz- und langfristige Ziele des Rehabilitationsprozesses werden im Team abgesprochen und die entsprechenden Interventionen geplant. Die Beurteilungen der Teammitglieder fließen in den schriftlichen Rehabilitationsplan ein. Der Rehabilitationsplan wird regelmäßig überprüft und angepasst.

\subsubsection{Interdisziplinäre Kooperation in der Rehabilitation}

In einer spezialisierten Rehabilitation, bei der es um die Behandlung mehrerer Beeinträchtigungen und der mit diesen einhergehenden Funktionsverluste geht, kann die enge Zusammenarbeit zwischen medizinischen Fachbereichen erforderlich sein. Die Experten der verschiedenen Fachgebiete müssen sich auf eine gemeinsame Strategie einigen, die sämtliche Interventionen $\mathrm{zu}$ den richtigen Zeitpunkten einschließt, und dürfen sich nicht auf isolierte Behandlungen in Ad-hoc-Manier zu verlassen. Um ein gemeinsames Vorgehen im Rahmen der übergreifenden Behandlungsstrategie zu erreichen, sind regelmäßige persönliche Kontakte zwischen den Vertretern der verschiedenen medizinischen Fachgebieten unabdingbar.

PRM-Fachärzte sollten in die Akutrehabilitation zum frühestmöglichen Zeitpunkt am besten schon während der Intensivbehandlung eingebunden werden [51]. Umgekehrt kann in Abteilungen zur Akutrehabilitation auch die kontinuierliche Mitarbeit von Spezialisten anderer medizinischer Fachgebiete nötig sein, die sich am Rehabilitationsprozess mit ihren eigenen fachspezifischen Interventionen beteiligen (z. B. Schließen einer Tracheotomie bei Patienten, die mit einer Beatmungshilfe behandelt wurden). Auch in späteren Rehabilitationsphasen und bei der Rehabilitation von Menschen mit lange dauernden Behinderungen ist eine enge Kooperation mit dem Primärbehandlungsarzt und anderen Fachärzten erforderlich.

Bei der Betreuung des Patienten muss schon zu einem frühen Zeitpunkt klar gestellt werden, wer den Behandlungsverlauf leitet. Dies wird sich zwar mit den Behandlungsfortschritten immer wieder ändern, doch sobald der Schwerpunkt auf Rehabilitation anstatt auf Reanimation oder medizinischer bzw. chirurgischer Akutbehandlung liegt, sollte der PRM-Facharzt den Entscheidungsprozess leiten.

\subsection{Physikalische und Rehabilitative Medizin in unterschied- lichen Phasen des Rehabilitationsprozesses \\ 4.4.1 Physikalische und Rehabilitative Medizin \\ in der akuten und postakuten Phase}

Die Rehabilitation in der frühen Phase der Wiederherstellung nach schwerer Krankheit oder größeren Verletzungen wird durch eine für den Patienten angenehmen Umgebung, in der auch seine Ängste und Sorgen berücksichtigt werden, wesentlich unterstützt. Während der ersten Wochen kann die Fähigkeit des Patienten, sich anhaltend auf ein Trainingsprogramm oder auf körperliche Bewegung zu konzentrieren, noch deutlich reduziert sein. Allerdings wurde beobachtet, dass bereits die einfache Verlegung eines Patienten mit Gehirnschädigungen aus einer geschäftigen chirurgischen oder neurochirurgischen Station in eine ruhigere Rehabilitationsstation oftmals bereits einen therapeutischen Nutzen mit sich bringen kann, der sich durch gesteigerte patientenseitige Aufmerksamkeit und Wahrnehmung sowie durch reduzierte Unruhe bemerkbar macht. Diese Effekte sind nicht nur an sich sehr wichtig, sondern auch eine wesentliche Voraussetzung für ein optimales Ansprechen des Patienten auf die Rehabilitation [52]. Vielfach stoßen Rehabilitationsteams, in denen sich Fachleute aus verschiedenen Bereichen um die Behandlung der Patienten mit komplexen Bedürfnissen bemühen, durch die angespannte Atmosphäre einer allgemeinen Krankenstation auf Schwierigkeiten. Fällt die Behandlung aus und wird versucht, Symptome wie z. B. Ängstlichkeit und Unruhe medikamentös zu behandeln, kann es zwar kurzzeitig zu einer Linderung der Symptome kommen; die Wiederherstellung der für eine Rehabilitation unabdingbaren kognitiven Funktionen wird jedoch verzögert.

Die folgenden Fallberichte veranschaulichen die Art von Problemen, denen PRM-Fachärzte gegenüberstehen. Die Entstehung sekundärer Komplikationen nach einer Krankheit oder schweren Verletzung kann durch frühe Interventionen vermieden werden.

Fallbericht 1: Infolge eines Verkehrsunfalls hatte ein 25-jähriger Mann ein schweres Schädel-Hirn-Trauma erlitten. Seine Symptome waren u.a. Verwirrtheit, Desorientiertheit, Agitation und Schluckstörungen. Demnach bestand ein schweres Risiko für eine lebensbedrohliche Aspirationspneumonie, welche die Wiederherstellung seiner Gehirnfunktion beeinträchtigt hätte. Darüber hinaus entwickelte er innerhalb kurzer Zeit Kontrakturen der unteren Extremitäten und eine Spastizität.

Durch eine entsprechende koordinierte Rehabilitation konnte eine ruhige Umgebung geschaffen werden, in der der Patient über seine Situation sprechen und diese verstehen konnte. Die Behandlung beruhte auf Verhaltensmanagement zum Abbau der Ängstlichkeit. Zur Vorbeugung einer Aspirationspneumonie und zur Sicherung einer adäquaten Ernährung wurde dem Patienten eine PEG-Sonde (perkutane endoskopische Gastrostomie) angelegt. Die Behandlung seiner Kontrakturen umfasste die Reduktion der Spastizität durch physikalische Therapie und die Anwendung dynamischer Schienen. Nach mehreren Monaten intensiver Rehabilitation konnte der Patient mit verbessertem Verhalten aus dem Krankenhaus entlassen werden. Auch seine Schluckunfähigkeit besserte sich, sodass er wieder normal essen und die PEG-Sonde entfernt werden konnte. Er begann wieder zu gehen und konnte in späterer Folge erneut einer beruflichen Tätigkeit nachgehen. 
Fallbericht 2: Einem 52-jährigen Mann mit Typ-2-Diabetes-mellitus und einem gangränösen Fuß musste das Bein ab dem Schienbein amputiert werden. Im Zuge der präoperativen Aufklärung erhielt der Patient Hinweise zur besseren Bewältigung der bevorstehenden Veränderungen an seinem Körper und in seinem Leben. Diese umfasste auch Anweisungen für den Umgang mit den sensorischen Veränderungen, dem veränderten Körperselbstbild und der neuen Gleichgewichtssituation, die ihn auf die Rehabilitation vorbereiten sollten.

Physiotherapeutische Maßnahmen wurden bereits in der frühen postoperativen Phase mit einer Atmungsstherapie sowie Prophylaxemaßnahmen von Thrombosen und Kontrakturen eingeleitet. Durch Verbände wurde auf die Bildung eines funktionellen Amputationsstumpfes und die Reduktion etwaiger Stumpfödeme geachtet. Seine ersten Gehversuche nach der Operation machte der Patient mit einer provisorischen Prothese, während sein Bein im Hinblick auf eine Dauerprothese vermessen wurde. Gleichzeitig wurden mit dem Patienten der Grad und die Art seiner körperlichen Anforderungen und Ziele besprochen, wobei seine Bedürfnisse hinsichtlich der etwaigen Anpassung seiner Wohnung, seines Arbeitsplatzes und seines Pkws ermittelt wurden. In diesem Zusammenhang wurden auch Faktoren wie z. B. der Weg vom Parkplatz zum Arbeitsplatz, die am Arbeitsplatz zurückzulegenden Wege und andere relevante Faktoren wie z.B. Freizeit- und Familienaktivitäten untersucht. Dem Patienten wurde gezeigt, wie er mit dem Stumpf und den Prothesen umzugehen hätte. Drei Monate nach der Amputation konnte er die Pflege seines Stumpfes einschließlich der Überwachung des Restgliedes selbstständig übernehmen. Er konnte seiner Arbeit wieder nachgehen, wird jedoch für den Rest seines Lebens in PRM-ärztlicher Betreuung bleiben.

Fallbericht 3: Eine 70-jährige unter Hüftgelenksarthrose leidende Frau erhielt eine Hüfttotalendoprothese. $\mathrm{Zu}$ diesem Zeitpunkt konnte sie weder gehen, baden noch eine Dusche benutzen. Sie konnte ihre Schuhe nicht anziehen und das Lenken eines Fahrzeugs war ebenfalls unmöglich. Das von der PRM-Abteilung geplante postoperative Programm umfasste intensive Physiotherapie und später Gymnastikübungen zu Hause, die es der Frau ermöglichen sollten, sich innerhalb und außerhalb des Hauses frei zu bewegen. Innerhalb weniger Wochen durfte die Frau wieder Auto fahren, und mithilfe ergotherapeutischer Maßnahmen konnte sie die Tätigkeiten des täglichen Lebens erneut bewältigen. Sie erhielt ein postoperatives Therapieprogramm, sodass sie dieses Programm zu Hause fortsetzen konnte. Von den Ergotherapeuten wurde ihre häusliche Umgebung überprüft, und es wurden Anpassungen vorgenommen, wodurch sie anschließend in der Lage war, wieder unabhängig zu leben.

\subsubsection{Rehabilitation bei stabiler, chronisch behindernder und progressiv voranschreitenden Erkrankungen}

Weitere Hauptarbeitsbereiche der PRM-Fachärzte sind die Erhaltung und Verbesserung der Funktion sowie die Vermeidung vorhersagbarer und vermeidbarer Komplikationen bei stabilen, chronisch behindernden sowie progressiv voranschreitenden $\mathrm{Er}$ krankungen. In einigen europäischen Ländern (z.B. Österreich, Deutschland, Italien und Polen) spielt die stationäre oder die in Tageskliniken angebotene Rehabilitation eine wichtige Rolle in der Behandlung chronischer Erkrankungen wie z. B. bei chronischen Krankheiten des Bewegungsapparats oder neuromuskulä- rer Störungen, bei chronischer Kreislauf-, Atemwegs- und Stoffwechselerkrankungen, bei Hautkrankheiten sowie bei urologischer und gynäkologischer Erkrankungen. Zur Behandlung funktioneller Störungen können auch mehrwöchige intensive Rehabilitationsmaßnahmen durchgeführt werden; diese kann sogar bis zu mehreren Jahren nach einem akuten Ereignis erfolgen [17].

Bei chronischen Erkrankungen zielen die Maßnahmen der Rehabilitation in erster Linie auf die Verbesserung der betroffenen Körperfunktionen und Steigerung der Aktivitäten ab. Derartige Maßnahmen umfassen auch Fragen der Teilhabe wie z.B. die berufliche Wiedereingliederung und die Vermeidung eines frühzeitigen krankheitsbedingten Berentung. Angewendet werden z. B. physikalische Therapien, Bewegungstherapie, Diät, psychologische Maßnahmen und Patientenschulung. Sowohl in offenen Verlaufsuntersuchungen (Follow-up-Studien) als auch in kontrollierten Studien [52], konnten die klinische Wirksamkeit und die positiven sozioökonomischen Auswirkungen aufgezeigt werden. Auch systematische multidisziplinäre Gesundheitsaufklärungsprogramme erwiesen sich als wirksam.

\subsection{Auswirkungen einer fehlenden Rehabilitation}

Das Rehabilitationspotenzial einer Person kann nicht unabhängig von der Situation betrachtet werden, die ohne Rehabilitation entstanden wäre. Die Frage, mit der sich die fachärztliche Rehabilitation befasst, lautet: „Wird der Patient aus einem Rehabilitationsprogramm einen Nutzen ziehen, den er nicht gehabt hätte, wenn man den Erholungsprozess sich selbst überlassen hätte?“ Der natürliche Verlauf einer Beeinträchtigung und die nachfolgenden Behinderungen und Funktionseinschränkungen spielen eine große Rolle im Ergebnis nach einer Rehabilitation. Bei manchen Krankheiten kommt es zu spontanen Erholungsvorgängen, sodass frühe Interventionen irrtümlich den Eindruck erwecken könnten, die Therapie sei wirksam gewesen [40,41]. Andererseits kann eine frühe Intervention zu einem besseren Ergebnis führen, selbst wenn eine volle Wiederherstellung nicht möglich ist [54].

Menschen mit dauerhaften Behinderungen und ihre Familien können durch die Rehabilitation eine bessere Lebensqualität erreichen; zu bedenken ist aber auch, dass sie bei fehlender Rehabilitation möglicherweise ein Leben in größerer Abhängigkeit und mit geringerer Lebensqualität hätten führen müssen [55]. In einem Akutkrankenhaus kann auf rehabilitationsfähige Störungen wie z.B. Ernährungs-, Schluck- und Mobilitätsprobleme sowie auf die Bereitstellung von Hilfsmitteln oft nicht eingegangen werden, weil der Behandlungsschwerpunkt unweigerlich auf den primären Krankheitszeichen liegt. Hier können PRM-Fachärzte bei der Vermeidung von Komplikationen helfen und dafür sorgen, dass ein optimales Funktionsniveau erzielt wird [56]. Bei ausbleibender Rehabilitation können Komplikationen und Funktionsverluste auftreten, sodass eine Entlassung aus dem Krankenhaus verzögert wird. Die Leistungserbringer im Gesundheitswesen sind gesetzlich dazu verpflichtet, allen Patienten die Rehabilitationsmaßnahmen zukommen zu lassen, die ihren gesundheitlichen Bedürfnissen entsprechen [57,58].

Durch das Fehlen von Rehabilitationsmaßnahmen können sich bei einer Reihe von Krankheiten schwerwiegende Probleme ergeben wie z.B.: 
Tab. 1 Grundlagen der Prävention

\begin{tabular}{|c|c|c|c|}
\hline Prävention & Merkmale & $\begin{array}{l}\text { Bereiche, in denen präventive } \\
\text { Maßnahmen ergriffen werden }\end{array}$ & Beispiele \\
\hline primäre & Vorbeugung von Krankheit und Verletzung & $\begin{array}{l}\text { politische und gesellschaftliche } \\
\text { Bereiche } \\
\text { primäre Betreuung }\end{array}$ & $\begin{array}{l}\text { Geschwindigkeitsbeschränkungen zur Vorbeugung } \\
\text { von Unfällen im Straßenverkehr } \\
\text { Verringerung von Risikofaktoren für kardiale und } \\
\text { zerebrovaskuläre Erkrankungen }\end{array}$ \\
\hline sekundäre & $\begin{array}{l}\text { Vermeidung der Auswirkungen und } \\
\text { Komplikationen von Krankheiten } \\
\text { oder Verletzungen an sich }\end{array}$ & $\begin{array}{l}\text { Akutkrankenhaus und Abteilung } \\
\text { für Frührehabilitation }\end{array}$ & $\begin{array}{l}\text { Vorbeugung intrakranieller Hypertension bei Gehirn- } \\
\text { schädigung } \\
\text { Vorbeugung von Schlaganfällen nach Myokardinfarkt } \\
\text { Vorbeugung von Immobilität und von Problemen } \\
\text { der Lebensfähigkeit des Gewebes } \\
\text { Vorbeugung von Kontrakturen }\end{array}$ \\
\hline tertiäre & $\begin{array}{l}\text { Vorbeugung der Auswirkungen von Krank- } \\
\text { heiten oder Verletzungen auf die Lebens- } \\
\text { führung des Patienten, d. h. Einschränkungen } \\
\text { der Aktivität und/oder Teilhabe }\end{array}$ & $\begin{array}{l}\text { postakute und Erhaltungsrehabili- } \\
\text { tationsabteilungen }\end{array}$ & $\begin{array}{l}\text { Behandlung von Verhaltensstörungen nach Gehirn- } \\
\text { schädigung } \\
\text { Vorbeugung vermeidbarer finanzieller Schwierigkeiten } \\
\text { und Arbeitslosigkeit nach Krankheit oder Verletzung }\end{array}$ \\
\hline
\end{tabular}

- Immobilität einschließlich Muskelschwäche, kardiorespiratorische Insuffizienz, Muskelatrophie, Dekubitalulzera, Spastizität, Kontrakturen und Osteoporose,

- Schmerzen,

- Ernährungsstörungen,

- Schluckbeschwerden,

- Blasen- und Darmprobleme (Verstopfung u. Inkontinenz),

- Kommunikationsstörungen,

- kognitive Probleme und die Unfähigkeit, aus dem Gelernten einen Nutzen zu ziehen,

- Stimmungs- und Verhaltensstörungen,

- Krankheit und systemische Erkrankungen aus einer Reihe von Ursachen wie z. B. Harnwegsprobleme, Herz- und Atemwegsprobleme, Diabetes mellitus,

- Komplikationen der Grunderkrankung.

Auch bei Wiedereingliederung des Patienten in das Alltagsleben sollten die Maßnahmen der Physikalischen und Rehabilitativen Medizin im Rahmen einer Nachbetreuung fortgesetzt werden, z. B. um Folgendes zu vermeiden:

- sekundäre Gesundheitsprobleme und soziale Isolation,

- Überlastung der Pflegepersonen und in der Folge Zusammenbruch der häuslichen Pflegesituation,

- unnötige Inanspruchnahme von praktischen Ärzten oder Sozialarbeitern,

- erneute Notaufnahme ins Krankenhaus,

- unnötige Unterbringung in Wohn- oder Pflegeheimen [8],

- unnötige und nicht angepasste Verschreibung von Hilfsmitteln,

- Unfähigkeit, die Hilfsmittel im Hinblick auf den technischen Fortschritt (z. B. Neuroprothesen) auf dem neuesten Stand zu halten.

Insgesamt kann das Fehlen einer Rehabilitation bedeuten, dass eine Person mit eingeschränkter Funktionskapazität und Lebensqualität zurückbleibt. Für die Gemeinschaft bedeutet dies eine Verschwendung von Ressourcen.

\subsection{Prävention}

\subsubsection{Förderung der Gesundheit}

Jedes Gesundheitssystem beruht auf dem Grundsatz der Gesundheitsförderung. Für alle medizinischen Berufe stellt daher die Prävention von Krankheiten und ihren Auswirkungen sowie das Vermeiden von Komplikationen ein wesentlicher Bestandteil ihrer Arbeit dar. Im Kontext der Rehabilitation beschäftigt sich die Krankheitsprävention nicht nur damit, den Eintritt und die Entwicklung einer Krankheit zu verhindern, sondern sie befasst sich mit den vielfältigen Möglichkeiten, wie die Folgen einer Krankheit auf alle Lebensbereiche des Patienten minimiert werden können. Daher wird die Krankheitsprävention gemäß den in Tab. 1 angeführten Grundlagen in primäre, sekundäre und tertiäre Prävention eingeteilt.

PRM-Fachärzte leisten auf allen Ebenen der Krankheits- und Verletzungsprävention einen Beitrag. $\mathrm{Zu}$ berücksichtigen sind die allgemeinen Grundlagen der körperlichen Bewegung einschließlich kardiovaskulärer Leistung, Leistung des Bewegungsapparats und der Koordinationsfähigkeit. Dies kann auch zur Vorbeugung von Bluthochdruck, Myokardinfarkt, Rückenschmerzen und Stürzen beitragen.

\subsubsection{Prävention sekundärer Komplikationen}

Bei Krankheiten oder Traumata kommt es häufig zu sekundären Komplikationen allgemeiner Art wie z.B. Lungenentzündung, Thrombosen, Dekubitalulzera, Dekonditionierung des Kreislauf und der Muskeln, Osteoporose und Anorexie. Hinzu kommen krankheitsspezifische Komplikationen wie z.B. Dysphagie nach Schlaganfall oder Miktionsstörungen bei Verletzungen des Rückenmarks. Alle diese Komplikationen können in PRM-Abteilungen erfolgreich behandelt werden.

\subsubsection{Auswirkungen der tertiären Prävention}

Es gibt mittlerweile umfangreiche Nachweise dafür, dass die Rehabilitation nicht nur sofortige Effekte hat [59-62] und eine Verbesserung der funktionellen Leistung und gute Ergebnisse hinsichtlich der Teilhabe mit sich bringt, sondern auch längerfristige Vorteile hat [63]. Menschen, die nach einer Krankheit oder Verletzung Rehabilitationsmaßnahmen erhalten haben, erleiden weniger oft einen vorzeitigen Tod bzw. müssen sich weniger häufig in Pflegeeinrichtungen begeben. Dies wurde in vielen Krankheitsgruppen und in allen Altersgruppen festgestellt, wenngleich jüngere Betroffene bessere Erholungsprozesse haben. In den meisten Studien wird die Bedeutung der Rehabilitation in zweierlei Hinsicht bestätigt. Erstens betreffen die meisten 
dokumentierten Verbesserungen das funktionelle Ergebnis, und zweitens haben Menschen mit Behinderungen, die in Rehabilitationszentren behandelt werden, weniger oft vermeidbare Komplikationen. Es gibt weniger körperliche Probleme (wie z.B. jene im Zusammenhang mit Immobilität, Kontrakturen und Dekubitalulzera) und auch weniger psychologische Probleme wie z.B. unbehandelte Depressionen. Obwohl zunächst nur gezeigt werden konnte, dass eine intensive Rehabilitation nach einem akuten Ereignis wie z.B. einer Kopf- oder Rückenmarkverletzung eindeutige kurzfristige Effekte hat [44], konnte inzwischen auch nachgewiesen werden, dass derartige kurzfristige Besserungen bei fehlender langfristiger Unterstützung wieder verloren gehen $[51,64]$. Sogar in Fällen, in denen die Wirksamkeit einzelner therapeutischer Modalitäten nicht nachzuweisen ist, zeigt sich, dass eine multidisziplinäre Rehabilitation nachweislich wirksam ist $[65,66]$. Um auch die spätere Entstehung vermeidbarer Komplikationen zu verhindern, ist es von großer Bedeutung, längerfristig mit den Betroffenen in Kontakt zu bleiben und so lange geeignete Rehabilitationsmaßnahmen durchzuführen, bis der natürliche Erholungsprozess abgeschlossen ist.

\section{Das Fachgebiet der Physikalischen und Rehabilitativen} Medizin

\subsection{Beitrag des Facharztes für Physikalische und Rehabilitative Medizin zum Rehabilitationsprozess}

Fachärzte für Physikalische und Rehabilitative Medizin schaffen die Rahmenbedingungen für eine gut organisierte rehabilitative Versorgung. Es konnte gezeigt werden, dass eine solche koordinierte Rehabilitation effektiver und nicht teurer ist als die Durchführung von Einzelmaßnahmen. PRM-Fachärzte berücksichtigen dabei in besonderem Maße die notwendige Langzeitversorgung. So kann eine Verbesserung der körperlichen und kognitiven Funktion durch Gehirnschädigung z.B. mehrere Jahre in Anspruch nehmen. Die PRM-Fachärzte stellen nicht nur sicher, dass sich der Patient in einer optimalen Umgebung befindet, in der die bestmögliche funktionelle Verbesserung erzielt werden kann, sondern sie arbeiten auch bei entsprechender Indikation über längere Zeit mit dem Patienten, damit er seine persönliche Funktionsfähigkeit und gesellschaftliche Teilhabe verbessern kann.

Eine besonders wichtige Rolle spielt der PRM-Facharzt in der Rehabilitation, wenn:

- eine Kombination von mehreren Beeinträchtigungen vorliegt, wie z.B. kognitive, Verhaltens- und körperliche Beeinträchtigungen, d.h. Situationen, für deren umfassende Analyse der PRM-Arzt speziell geschult ist und in die er auch die Expertise der nichtärztlichen Therapeuten einbezieht;

- es sich um eine gravierende, zu Aktivitäts- und Teilhabeverlusten führende Beeinträchtigung handelt, die durch ein plötzliches Ereignis wie z.B. einen Schlaganfall, eine Rückenmarkverletzung oder ein traumatisches Ereignis hervorgerufen worden ist;

- es sich um eine Grunderkrankung handelt, die schubweise verläuft, wie z.B. multiple Sklerose oder rheumatoide Arthritis;

- medizinische Maßnahmen zur Verfügung stehen, die eine direkte Milderung der Beeinträchtigungen herbeiführen bzw. die das Wohlbefinden und die Aktivität verbessern können, wie z. B. bei Arzneimitteln zur Behandlung von Spastizität, Inkontinenz oder Schmerzen;

- die medizinische Behandlung der Grunderkrankung und deren Komplikationen selbst ein Risiko beeinträchtigender Nebenwirkungen in sich bergen, die der Überwachung bedürfen;

- die medizinischen Risiken einer beeinträchtigenden Krankheit durch Veränderungen in der Lebensführung des Patienten noch weiter verstärkt werden können, z. B. beim Übergang vom jugendlichen zum Erwachsenenalter, beim Übergang von der Schulausbildung zum Erwerbsleben und bei den Alterungsprozessen im späteren Leben.

PRM-Fachärzte können sich ausschließlich auf den Bereich der Rehabilitation konzentrieren, ihre Arbeit wird nicht durch die Erfordernisse der medizinischen Akutversorgung beeinträchtigt. Es ist bekannt, dass eine separate PRM-Abteilung in Kliniken für den Patienten von Vorteil ist [18]. Den Patienten treffen kompetente Fachärzte an, die eine einschlägige Ausbildung absolviert haben und sich zur Gänze dieser Tätigkeit widmen. Patienten mit schweren körperlich behindernden Erkrankungen und insbesondere jene mit komplexem Rehabilitationsbedarf brauchen die Betreuung durch ein multidisziplinäres Team unter der Leitung eines PRM-Facharztes (s. Kap. 4).

\subsection{Das Fachgebiet Physikalische und Rehabilitative Medizin in Europa}

Fachärzte für Physikalische und Rehabilitative Medizin haben in Europa eine mindestens vierjährige Facharztweiterbildung [67], die je nach den nationalen Ausbildungsrichtlinien für die postgraduelle medizinische Ausbildung bis zu 6 Jahren betragen kann. Die Facharztweiterbildung bezieht sich nicht auf die Behandlung eines bestimmten Organs oder bestimmter Krankheiten, sondern konzentriert sich auf funktionelle Probleme, die infolge unterschiedlicher Krankheiten auftreten. PRM-Fachärzte bieten eine ärztliche Betreuung für Menschen mit Behinderungen infolge chronischer Krankheiten und Traumata sowie sonstiger schwerwiegender gesundheitlicher Probleme an. Das Hauptziel der PRM-ärztlichen Betreuung ist die Verbesserung körperlicher und mentaler Funktionen, sodass es dem Patienten möglich wird, aktiv zu sein, seine Lebensqualität zu verbessern und am gesellschaftlichen Leben teilnehmen zu können. PRM-Fachärzte können aber auch die Grunderkrankung behandeln. Bei anhaltenden Symptomen und Beschwerden haben sie die Möglichkeit, Funktionsfähigkeit, Aktivität und Teilhabe durch spezialisierte Rehabilitationsmaßnahmen zu fördern. Somit können sie Menschen mit Erkrankungen, die zu Funktionsdefiziten führen (z.B. neurologische, sonstige Erkrankungen des Bewegungsapparates, Amputationen, Herz- und Lungenerkrankungen usw.) helfen, ein besseres Leben zu führen.

\subsection{Krankheiten und Funktionsstörungen, die in der Physikalischen und Rehabilitativen Medizin behandelt werden}

Fachärzte für PRM betreuen Patienten mit einer Vielzahl unterschiedlicher Grunderkrankungen (Anhang 3). Sie befassen sich mit den Auswirkungen dieser Erkrankungen auf die persönliche Funktionsfähigkeit und Teilhabe der Patienten. Unabhängig von der zugrunde liegenden Diagnose arbeiten PRM-Fachärzte immer an einer Besserung des Funktionszustands. Dennoch ist 
eine exakte Diagnose erforderlich, um eine Rehabilitationsprognose erstellen zu können und das Rehabilitationspotenzial zu beurteilen.

Unter den zahlreichen gesundheitlichen Störungen, mit denen PRM-Fachärzte tagtäglich konfrontiert werden, gibt es eine Reihe allgemeiner Probleme. Dazu gehören:

- längere Bettlägerigkeit und Immobilisierung, die für den Patienten Dekonditionierung und Verlust der körperlichen und psychologischen Funktionsfähigkeit bedeuten;

- motorische Defizite, die zu Schwäche und Verlust der persönlichen Funktionsfähigkeit führen;

- Spastizität, gefolgt von Deformitäten der Gliedmaßen und vermindertem Selbstwertgefühl;

- Blasen- und Darmfunktionsstörungen, die häufig bei Patienten mit Behinderungen auftreten;

- Dekubitalulzera als Folge der Immobilisierung bei Patienten mit Rückenmarksverletzungen, Diabetikern, dekonditionierten und älteren Patienten;

- Schluckstörungen (Patienten mit Dysphagie verlieren den Genuss am Essen und haben ein hohes Risiko einer Aspirationspneumonie und der Unterernährung);

- Schmerzsyndrome,

- Kommunikationsschwierigkeiten,

- sexuelle Probleme und sexuelle Dysfunktion, die sowohl Identitäts- und Selbstverständnisproblemen als auch Organfunktionsstörungen einschließen;

- Stimmungs-, Verhaltens- und Persönlichkeitsveränderungen sowie

- Änderungen im Bereich der Familiendynamik, der persönlichen Beziehungen, der beruflichen Chancen und der finanziellen Sicherheit.

Darüber hinaus können PRM-Fachärzte auch zur Rehabilitation von Patienten mit psychosomatischen, gynäkologischen und dermatologischen Erkrankungen beitragen.

\subsection{Diagnostik, Assessment und Evaluation}

Auch in der Physikalischen und Rehabilitativen Medizin muss vor Beginn der Behandlung und der problemorientierten Rehabilitationsmaßnahmen eine Diagnose gestellt werden. Darüber hinaus befassen sich PRM-Fachärzte mit allen Aspekten der Funktionsfähigkeit und Teilhabe, die für die Erfassung der Patientenprobleme und die Zielstellung der Rehabilitation wichtig sind. Dies erfolgt gemeinsam mit der betroffenen Person, ihrer Familie und den Mitgliedern des Rehabilitationsteams. Die diagnostischen Maßnahmen und Assessments umfassen alle Dimensionen der Körperfunktionen und -strukturen sowie alle für den Rehabilitationsprozess relevanten Fragen bezüglich Aktivität und Teilhabe. Darüber hinaus werden relevante Kontextfaktoren erhoben. Bei der Anamnese werden alle ICF-Dimensionen berücksichtigt.

Um die für die Krankheit und den Rehabilitationsprozess maßgeblichen strukturellen Defizite diagnostizieren zu können, werden zusätzlich zur körperlichen Untersuchung die üblichen Untersuchungsmethoden und -techniken angewendet. Dazu gehören Laboranalysen von Blutproben, bildgebende Verfahren u.a.
Ein wesentlicher Teil der PRM-Diagnostik besteht in der klinischen Beurteilung und Messung der Funktionseinschränkungen und des Funktionspotenzials im Hinblick auf den Rehabilitationsprozess. Dies beinhaltet u.a. die klinische Untersuchung der Muskelkraft und des Bewegungsumfangs sowie der Kreislauf- und Atemfunktion. Zu den technischen Messungen können Muskelfunktionsuntersuchungen (Kraft, elektrische Aktivität und andere), Untersuchungen der Kreislauffunktionen (Blutdruck, Herzfrequenz, EMG in Ruhe und unter Belastung), Lungenfunktionsprüfung und sonstige Messungen gehören. Die PRM-Fachärzte verwenden standardisierte Methoden zur Messung der Leistungsfähigkeit wie z. B. Ganganalyse, isokinetische Muskeltests und sonstige Tests der Bewegungsfunktionen. Bei der Rehabilitation von Patienten mit bestimmten Erkrankungen werden besondere diagnostische Maßnahmen benötigt, z. B. eine Dysphagiediagnostik bei Schlaganfallpatienten, urodynamische Untersuchungen bei Patienten mit Rückenmarkverletzungen oder eine Analyse der übergeordneten Funktionen bei Patienten mit Gehirnschädigung [69,70].

Zur Beurteilung der Aktivität der Patienten stehen zahlreiche Methoden zur Verfügung. Die zwei wichtigen Methoden sind:

- Standardisierte Testung bestimmter Einzelfunktionen (z.B. Gehtest, Greiftest oder Umgang mit Werkzeugen, Durchführung von Tätigkeiten in einem standardisierten Arbeitsumfeld). Die Tests werden einerseits qualitativ (durch PRM-Fachärzte oder entsprechend ausgebildete Therapeuten) und andererseits quantitativ beurteilt (Zeit zur Durchführung der Tätigkeit, die Fähigkeit, bestimmte Lasten zu heben usw.).

- Beurteilung komplexer Aktivitäten wie z.B. Tätigkeiten des täglichen Lebens (sich waschen, sich anziehen, zur Toilette gehen usw.) und der Leistungsfähigkeit im täglichen Leben (Gehen, Sitzen etc.). Die Befragungen können durch Ärzte und Therapeuten, aber auch mittels standardisierter Fragebogen durch die Betroffenen selbst vorgenommen werden.

Die Analyse der Teilhabe erfolgt in erster Linie im Rahmen von Interviews mit dem Patienten und mithilfe standardisierter Fragebögen. Zur Evaluierung gesellschaftlicher oder mit dem Berufsleben zusammenhängender Probleme der Teilhabe werden sozioökonomische Parameter (z.B. Anzahl der Tage im Krankenstand) herangezogen.

Zahlreiche PRM-Beurteilungsinstrumente beruhen auf einer Kombination von Parametern der Körperfunktionen, der Aktivitäten und der Teilhabe. Sie dienen als Entscheidungsgrundlage für die Indikationsstellung von Rehabilitationsmaßnahmen (Zuweisungen) bzw. zur Beurteilung der Ergebnisse der Intervention (Evaluierung). Die geeigneten Instrumente werden unter Berücksichtigung der Krankheitsbilder, der Funktionsprobleme des einzelnen Patienten und der Phase des Rehabilitationsprozesses ausgewählt [71].

Zur Beurteilung der sozialen und physikalischen Kontextfaktoren werden Interviews oder standardisierte ICF-Checklisten verwendet. Persönliche Faktoren, wie z.B. die Bewältigungsstrategien des Patienten, werden anhand standardisierter Fragebögen erhoben.

Zur Beurteilung der allgemeinen und spezifischen funktionellen Leistungsfähigkeit sowie des Rehabilitationsprozesses stehen 
Tab. 2 Beispiele einiger der in einem Rehabilitationsplan behandelten Probleme

Problem Ziele mögliche Interventionen

\section{Körperfunktionen und Aktivitäten}

körperliche Aktivität

Kommunikation

Verhalten u. Stimmung

Schmerzen

Inkontinenz

Unfähigkeit der Selbstpflege

Erreichen der Fähigkeit, sich selbst waschen, pflegen,

\section{Teilhabe}

Unfähigkeit, zu Hause alleine zurechtzukommen

Verlust des Arbeitsplatzes anziehen und selbstständig zur Toilette gehen zu können.

Steigerung der Mobilität innerhalb und außerhalb der eigenen Wohnung (Erreichen/Verbessern des Gehens, Treppensteigens, privater Fahrzeuge, öffentlicher Verkehrsmittel)

Verbesserung von Kommunikation, Sprechen und Verstehen

Verhaltensstrategie

Normalisierung der Stimmung

Linderung von Schmerzen

Förderung der Blasen- und Darmkontinenz

Wiedererlernen der Zubereitung von Mahlzeiten und der Haushaltsführung

Rückkehr zum Arbeitsplatz
Haltungs- und Bewegungstraining mit den erforderlichen Hilfsmitteln

Haltungs- und Bewegungstraining ohne Hilfsmittel Anpassen von und Üben mit Hilfsmitteln zur Förderung der Beweglichkeit

Training unter der Regie von Sprech- und Sprachtherapeuten zur Verbesserung von Sprache und von Sprechfähigkeit Anpassen von und Üben mit unterstützenden technischen Hilfsmitteln, falls erforderlich

medikamentöse Behandlung, Psychotherapie, Verhaltenstherapie und Patientenschulung

Verminderung von Depressionen und Ängstlichkeit durch Aufklärung und medikamentöse Behandlung

Analgetika, physikalische Therapien, Stressmanagement, Anwendung von Bewältigungs- und anderen Strategien

Wiedererlernen der Blasenkontrolle, Beckenbodengymnastik, medikamentöse Behandlung, Verwendung von Kathetern und Stomata

Analyse der einzelnen Komponenten von Aktivitäten, Wiederherstellung bestimmter Fähigkeiten mit Hilfe von alternativen Methoden und/oder Hilfsmitteln und/oder das Wiedererlernen solcher Fähigkeiten

Analyse der einzelnen Komponenten von Aktivitäten, Wiederherstellung bestimmter Fähigkeiten mit Hilfe von alternativen Methoden bzw. Hilfsquellen und/oder Hilfsmitteln und/oder das Wiedererlernen solcher Fähigkeiten

Analyse der einzelnen Komponenten der Aktivitäten und der Rückkehr zum Arbeitsplatz, Wiederherstellung von Fähigkeiten, Anpassungen der Arbeit, Wiedereinschulung, Anpassungen des Arbeitsplatzes und der Hilfsmittel, Verbesserung des Zugangs und der Unterstützung am Arbeitsplatz zahlreiche Diagnoseverfahren zur Verfügung [72]. Einige überschneiden sich mit einzelnen ICF-Komponenten. So umfassen beispielsweise der Functional Independence Measure (Funktioneller Selbstständigkeitsindex - FIM) [73] und der Barthel-Index [74] Aspekte körperlicher Funktionen und Aktivitäten sowie einschlägige Begleiterkrankungen und das Ausmaß der benötigten Hilfe. Die Auswahl der Messmethoden hängt von der Rehabilitationsphase und den Zielen des Rehabilitationsprozesses sowie auch von der funktionellen Leistungsfähigkeit des einzelnen Patienten ab.

Anhang 5 enthält eine Liste der diagnostischen Methoden.

\subsection{Rehabilitationsplan}

In der Physikalischen und Rehabilitativen Medizin wird zur Steuerung der problemorientierten Rehabilitationsprozesse (Tab. 2) für jeden Patienten ein individueller Rehabilitationsplan erstellt und umgesetzt. An der Erstellung dieses Planes sind die Patienten, alle Mitglieder des Rehabilitationsteams, die den betreffenden Patienten betreuen aktiv teil. Der Schwerpunkt des Plans hängt von den jeweilig bestehenden Problemen ab, wenngleich er eine standadrisierte Grundstruktur besitzt. Der Plan muss vom Rehabilitationsteam regelmäßig überarbeitet und aktualisiert werden und dient den Mitgliedern des Teams als Kommunikationsbasis, um die Fortschritte des Patienten im Zuge der Rehabilitation beurteilen zu können.
Die PRM-Fachärzte sind für die Ausarbeitung des Rehabilitationsplans und die Festlegung des Zeitrahmens zur Durchführung der Maßnahmen verantwortlich. Folgende Informationen müssen im Plan enthalten sein:

- Diagnose;

- vorhandene Symptome und erhaltene Funktionen (gemäß ICF-Modell, siehe Kapitel 3.2);

- Rehabilitationsziele des Patienten;

- Rehabilitationsziele der Betreuer/der Familie;

- Rehabilitationsziele der einzelnen Mitglieder des Teams.

\subsection{Interventionen der Physikalischen und Rehabilitativen Medizin}

Die Physikalische und Rehabilitative Medizin bedient sich zahlreicher unterschiedlicher Interventionen. Basierend auf der gestellten Diagnose und der Analyse der funktionellen Einschränkungen des Patienten erstellen die PRM-Fachärzte einen Interventionsplan. Daraufhin führt entweder der Facharzt selbst und/ oder mehrere Teammitglieder die Intervention mit dem Ziel durch, die vorhandenen Probleme zu lösen. Wenn kein eigenenes Behandlungsteam zur Verfügung steht kann der PRM-Facharzt die Therapie auch verschieben. $\mathrm{Zu}$ den Interventionen zählen:

- Medizinische Interventionen

- medikamentöse Behandlung zur Wiederherstellung oder Verbesserung der Körperstrukturen und/oder -funktionen, 
z.B. Schmerztherapie, entzündungshemmende Therapie, Regulierung des Muskeltonus, Verbesserung kognitiver Funktionen, Verbesserung der körperlichen Leistungsfähigkeit, Behandlung der Depression;

- praktische Anwendungen einschließlich Injektionen und anderer Methoden zur Verabreichung von Medikamenten;

- Beurteilung und Überprüfung von Interventionen;

- Prognosestellung.

- Physikalische Therapien

- Manuelle Therapie bei reversibler Gelenksteifigkeit und damit zusammenhängenden Funktionsstörungen im Bereiche der Weichteile;

- Bewegungstherapie;

- Elektrotherapie;

- sonstige Behandlungen einschließlich Ultraschall, Wärmeund Kältebehandlungen, Fototherapie (z.B. Lasertherapie), Hydrotherapie und Balneotherapie, Diathermie, Massagetherapie und manuelle Lymphdrainage;

- Ergotherapie

- zur Analyse von Aktivitäten z.B. Tätigkeiten des täglichen Lebens und des Berufslebens, zur Unterstützung geschädigter Körperstrukturen (z.B. Schienen),

- um dem Patienten Lösungswege aufzuzeigen, mit denen er Barrieren bei Tätigkeiten des täglichen Lebens überwinden kann (z.B. Anpassung der persönlichen Einrichtungen),

- zum Üben von Fertigkeiten angesichts eingeschränkter Funktion und Kognition sowie

- zur Steigerung der Motivation;

- Sprech- und Sprachtherapie im Rahmen komplexer spezialisierter Rehabilitationsprogramme;

- Behandlung der Dysphagie;

- neuropsychologische Interventionen;

- psychologische Beurteilung und Interventionen, einschließlich Beratung;

- Ernährungstherapie;

- Hilfsmittel für behinderte Personen, assistive Technologien, Prothetik, Orthotik, technische Hilfen und Hilfsmittel;

- Patientenaufklärung;

- Rehabilitationspflege.

\subsection{Physikalische und Rehabilitative Medizin in der Praxis - klinische Tätigkeiten und Einrichtungen}

Das Tätigkeitsfeld der PRM-Fachärzte erstreckt sich auf alle Phasen der Rehabilitation und des Wiederherstellungsprozesses sowie auf die Betreuung von Patienten mit chronischen Erkrankungen. Sie praktizieren in den verschiedensten klinischen Einrichtungen. Diese reichen von Akutkrankenhäusern über eigenständige Rehabilitationszentren und PRM-Serviceabteilungen in Krankenhäusern bis hin zu gemeindenahen Rehabilitationszentren und selbstständigen Facharztpraxen. Auch wenn sich die Facharzttätigkeiten je nach klinischem Umfeld unterscheidet, so gelten in allen Bereichen die gleichen allgemeinen Grundsätze der PRM.

In Akutkrankenhäusern sind spezialisierte Rehabilitationseinrichtungen unentbehrlich. Ein Akutkrankenhaus sollte über Betten unter der Verantwortung eines PRM-Facharztes und über ein mobiles Rehabilitationsteam verfügen, das Patienten auf Intensiv- und anderen Akutstationen betreut und behandelt. Die PRM-
Fachärzte erstellen nicht nur Diagnosen und führen Assessments durch, sondern sind auch für die Behandlung der Patienten in ihren eigenen Rehabilitationsabteilungen und in anderen Krankenstationen zuständig. Durch seine konsiliarische Funktion trägt der PRM-Facharzt dazu bei, dass die Rehabilitation, die funktionelle Wiederherstellung und die Präventionsmaßnahmen gegen sekundäre Funktionsverluste, z.B. infolge Immobilisierung (wie z.B. Kontrakturen, Lungenentzündungen oder Thrombose) so früh wie möglich eingeleitet werden. Eine frühzeitige fachübergreifende Rehabilitation beugt langfristigen Funktionseinschränkungen vor und/oder bewirkt eine Reduktion derselben [61-63].

Bei Patienten mit Hirnschäden kann schon die Verlegung aus einer chirurgischen oder neurochirurgischen Station in eine ruhigere Rehabilitationsatmosphäre bereits einen therapeutischen Nutzen mit sich bringen, der sich durch eine Besserung von Aufmerksamkeit, Konzentration und kognitiver Funktonen bemerkbar macht [55]. Allgemeine Akutstationen sind für die Durchführung einer multidisziplinären Rehabilitation von Patienten mit komplexen Funktionsdefiziten nicht geeignet.

In Rehabilitationskliniken (einschließlich Tageskliniken) und Rehabilitationsabteilungen in Akutkrankenhäusern werden alle Patienten durch den PRM-Facharzt betreut. Dieser untersucht den Patienten, beurteilt seine Funktionsfähigkeit und schätzt den Einfluss der Umgebung auf seine Funktionsfähigkeit ab. Er bestimmt die notwendigen Interventionen wie z. B. physikalische Therapien, Psychotherapien, Ergotherapien, Sprechtherapien, neuropsychologisches Training, Medikamente und soziale Interventionen. Auch die Therapeuten untersuchen den Patienten vor Durchführung der Interventionsmaßnahmen. Die Ergebnisse der Untersuchungen des PRM-Facharztes und die funktionellen Beurteilungen der Therapeuten bilden die Basis des Rehabilitationsplans und künftiger Entscheidungen des RehabilitationsTeams.

Entscheidungen über die Entlassung der Patienten aus dem Krankenhaus obliegen dem PRM-Facharzt und werden in Teamkonferenzen besprochen, an denen auch die Person mit Behinderungen selbst und ihre Familienmitglieder aktiv teilnehmen. Die PRM-Fachärzte erstellen einen umfassenden Entlassungsbericht auf Basis der Untersuchungen und Informationen, die ihnen von den anderen Teammitgliedern zur Verfügung gestellt werden. Dieser Bericht enthält Informationen über die vorliegenden Erkrankungen, den funktionellen Zustand des Patienten, die Fähigkeit zur Durchführung von Aktivitäten und zur Teilhabe zum Zeitpunkt der Entlassung; er enthält auch prognostische Informationen und Empfehlungen zur weiteren pflegerischen Betreuung, Behandlung und Rehabilitation.

In Ambulanzen und Privatpraxen liegt der Schwerpunkt der PRM-Tätigkeit auf anderen Bereichen. Hier konzentrieren sich die Fachärzte auf die Diagnostik und die Einleitung der Behandlung. Nach Abschluss der Untersuchungen und des funktionellen assessments wird dem Patienten entweder eine einzelne Therapieserie (Physiotherapie, Ergotherapie oder andere) verschrieben oder es kommt ein Teamansatz zum Tragen (wenn eine multidisziplinäre Rehabilitation erforderlich ist). Im Anschluss an die Behandlungsserie wird der Patient erneut vom PRM-Facharzt 
untersucht. Dieser entscheidet dann über weitere Interventionen bzw. gegebenenfalls über eine Rücküberweisung zum primär behandelnden Arzt.

PRM-Fachärzte arbeiten in enger Kooperation mit dem Patienten und dessen Familie, wobei auch die Abstimmung mit dem behandelnden Arzt für Allgemeinmedizin und anderen Fachärzten von großer Wichtigkeit ist, insbesondere dann, wenn eine Diagnostik oder Therapie erforderlich ist, die sich mit anderen Fachgebieten wie Neurologie, Kardiologie oder orthopädische Chirurgie überschneidet.

Wenn notwendig arbeiten PRM-Fachärzte zusätzlich mit spezialisierten Rehabilitationsteams auf Gemeindeebene zusammen (z.B. mit Spezialteams für erworbene Gehirnschädigung, für chronische neurologische Erkrankungen, für Übergangsprobleme oder für Erkrankungen des Bewegungsapparates) und stehen diesen Rehabilitationsteams beratend zur Seite.

\section{Qualitätsstandards in der Physikalischen und Rehabilitativen Medizin}

\subsection{Theoretische und praktische Ausbildung 6.1.1 Facharztausbildung}

In allen Ländern Europas mit Ausnahme von Dänemark und Malta ist die PRM ein eigenständiges medizinisches Fachgebiet, auch wenn sich Name und Schwerpunkt entsprechend den einzelstaatlichen Traditionen und der Gesetzeslage unterscheiden (siehe Tab. 3). Je nach Land dauert die Fachausbildung zwischen vier und sechs Jahren [67] (UEMS-Charta über die Ausbildung, Richtlinie der EG 93/16/EWG, 5. April 1993). PRM-Fachärzte können sich innerhalb der Mitgliedsstaaten der UEMS frei bewegen bzw. ihren Beruf ausüben, benötigen jedoch ein Zeugnis ihrer nationalen Ausbildungsbehörde. Dieses Zeugnis ist auch die Voraussetzung dafür, vom European Board of PRM, von dem ein umfassendes System der postgraduierten Ausbildung für PRMFachärzte entwickelt wurde (siehe Details in Anhang 5), zertifiziert zu werden. Dieses System umfasst:

- ein Curriculum für die postgraduierten Ausbildung bzw. Weiterbildung, das die Grundlagen der PRM und ihre Anwendung bei bestimmten Erkrankungen enthält;

- einen standardisierten Weiterbildungsgang von mindestens vier Jahren in einer PRM-Abteilung, der in einem einheitlichen offiziellen Protokollbuch im Detail dokumentiert wird;

- eine für ganz Europa einheitliche jährliche schriftliche Abschlussprüfung;

- ein System nationaler Manager, die für die Qualität der Ausbildung und die Akkreditierung der Weiterbildungsstätten zuständig sind und über gute Kontakte zu den Auszubildenden ihres Landes verfügen;

- Standards für die Anerkennung als Ausbildner und für einen Zertifizierungsprozess;

- eine Qualitätskontrolle für Ausbildungsstätten auf der Basis von „Site visits“ durch speziell akkreditierte Fachärzte und

- die kontinuierliche berufliche Weiterentwicklung im Rahmen der UEMS, die ein medizinisches Fortbildungssystem mit einer Rezertifizierung und -akkreditierung in Abständen von 10 Jahren (siehe nachfolgenden Abschnitt 6.3) vorsieht.
Tab. 3 Name des Fachgebiets in den Mitgliedsstaaten der UEMS

\begin{tabular}{|c|c|}
\hline Land & Name des Fachgebiets \\
\hline Österreich & $\begin{array}{l}\text { Physikalische Medizin und allgemeine } \\
\text { Rehabilitation }\end{array}$ \\
\hline Belgien & $\begin{array}{l}\text { Fysiche Geneeskunde en Revalidatie } \\
\text { Médecine Physique et de Réadaptation }\end{array}$ \\
\hline Kroatien & Fizikalna medicine i rehabilitacija \\
\hline Zypern & 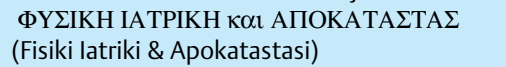 \\
\hline Tschechische Republik & Rehabilitační a fyzikální lékařství \\
\hline Dänemark & Fysiurgi* \\
\hline Estland & Taastusravi ja füsiaatria \\
\hline Finnland & Fysiatria \\
\hline Frankreich & Médecine Physique et de Réadaptation \\
\hline Deutschland & Physikalische und Rehabilitative Medizin \\
\hline Griechenland & 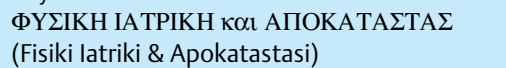 \\
\hline Ungarn & Fizioterapia es Rehabilitóciá \\
\hline Island & Endurhæfingarlækningar \\
\hline Irland & Rehabilitation Medicine \\
\hline Italien & Medicina Fisica e Riabilitativa \\
\hline Lettland & Medicīniskā Rehabilitācija \\
\hline Litauen & Fizine medicina ir reabilitacija \\
\hline Luxemburg & Médecine Physique et de Réadaptation \\
\hline Malta & - \\
\hline Niederlande & Revalidatie Geneeskunde \\
\hline Norwegen & Fysikalsk medisin og rehabilitering \\
\hline Polen & Medycyna fizykalna i rehabilitacja \\
\hline Portugal & Medicina Física e de Reabilitação \\
\hline Rumänien & Medicinā Fizicā şi de Recuperare \\
\hline Serbien und Montenegro & Fizikalna Medicina I Rehabilitacija \\
\hline Slowakei & Fyziatria, balneológia \& liečebná rehabilitácia \\
\hline Slowenien & Fizikalna in rehabilitacijska medicina \\
\hline Spanien & Medicina Fisica y Rehabilitación \\
\hline Schweden & Rehabiliterings Medizin \\
\hline Schweiz & $\begin{array}{l}\text { Médecine Physique et de Réadaptation } \\
\text { Physikalische Medizin und Rehabilitation }\end{array}$ \\
\hline Türkei & Fiziksel Tip ve Rehabilitasyon \\
\hline Vereinigtes Köniqreich & Rehabilitation Medicine \\
\hline
\end{tabular}

* obwohl in Dänemark derzeit keine Facharztweiterbildung existiert, arbeiten dort Fachärzte der PRM

Weitere Informationen über dieses Aus- und Fortbildungssystem sowie Anmeldungsformulare sind auf der Website der Sektion: www.euro-prm.org erhältlich.

Derzeit gibt es in Europa 10280 PRM-Fachärzte, von denen ca. 2000 eine Zertifizierung durch das European Board besitzen und 2800 sich in Ausbildung befinden. Eine Liste der 70 vom PRM-Board anerkannten Ausbildungsstätten steht auf der Website (s. o.) zur Verfügung [76]

\subsubsection{Ausbildung im Rahmen des Medizinstudiums}

Die UEMS-Sektion erhebt zurzeit Daten über die PRM-Ausbildung für Medizinstudenten und wird in nächster Zukunft darüber berichten. Für einen systematischen Ansatz müssen folgende Grundsätze beachtet werden:

Jeder Patient kann, unabhängig davon, in welcher Einrichtung des Gesundheitssystems (z.B. Krankenhaus, allgemeine Praxis, Pflegeeinrichtung) er sich befindet, Rehabilitation benötigen. Aus diesem Grund besteht für alle Ärzte die Notwendigkeit, sich ein Basiswissen über die Rehabilitation anzueignen, auch wenn die meisten nicht als Fachärzte auf diesem Gebiet tätig sein oder bestimmte Rehabilitationsmaßnahmen durchführen werden. Daher ist es von besonderer Wichtigkeit, dass in allen medi- 


\begin{tabular}{c|c|c}
$\begin{array}{c}\text { Fertigkeiten } \\
\text { des } \\
\text { Teilgebietes }\end{array}$ & $\begin{array}{c}\text { Fertigkeiten } \\
\text { des } \\
\text { Teilgebietes }\end{array}$ & $\begin{array}{c}\text { Fertigkeiten } \\
\text { des } \\
\text { Teilgebietes }\end{array}$ \\
$\begin{array}{c}\text { (z.B. Rehabilitation } \\
\text { von Patienten } \\
\text { mit Rückenmark- } \\
\text { verletzungen) }\end{array}$ & $\begin{array}{c}\text { (z.B. Rehabilitation } \\
\text { von Patienten } \\
\text { mit Störungen des } \\
\text { Bewegungs- } \\
\text { apparates und } \\
\text { nach Amputation) }\end{array}$ & $\begin{array}{c}\text { (z.B. Physikalische } \\
\text { Medizin oder } \\
\text { Sportmedizin) }\end{array}$ \\
\hline
\end{tabular}

Abb. 3 Grundschema der Erlangung von

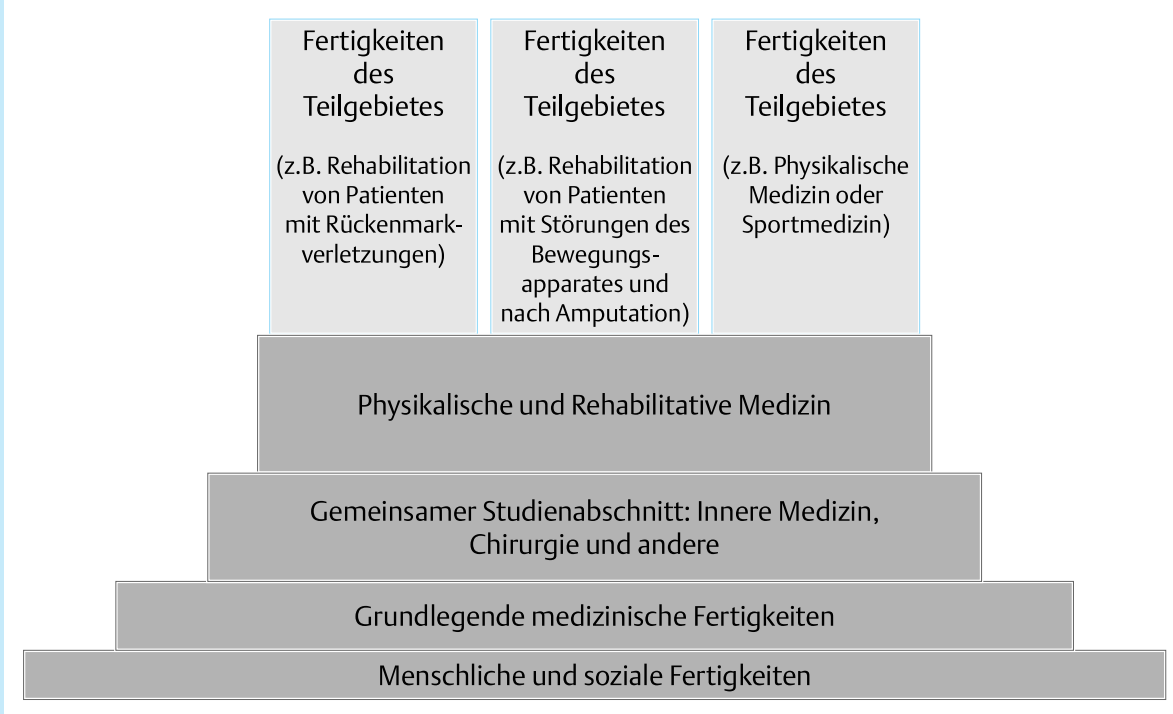

Kompetenzen im Fachgebiet PRM.

zinischen Fakultäten, in denen Medizinstudenten ausgebildet werden, gut ausgebildete PRM-Fachärzte das Fach der PRM unterrichten, wobei die Darstellung folgender Themen als Mindestanforderung anzusehen ist:

- Grundlagen der Rehabilitation und das biopsychosoziale Modell der internationalen Klassifikation der Funktionsfähigkeit, Behinderung und Gesundheit;

- Organisation und praktische Anwendung des Rehabilitationssystems (Akut- und Postakutrehabilitation sowie Rehabilitationsprogramme für Patienten mit chronischen Erkrankungen);

- Grundlagen und Möglichkeiten der Physiotherapie, Ergotherapie und anderer Rehabilitationstherapien;

- umfassende Rehabilitationsprogramme und ihre Hauptindikationen;

- Rehabilitationsbedürfnisse von Patienten mit besonderen Erkrankungen (z.B. Schlaganfall, multiples Trauma, Kreuzschmerzen, Arthritis, Krebserkrankung usw.);

- Kenntnis des Sozialsystems und der Gesetzgebung hinsichtlich Behinderung und Rehabilitation auf nationaler Ebene sowie Kenntnis ethischer Aspekte und der Menschenrechte im Zusammenhang mit der Rehabilitation.

In einigen europäischen Ländern (Belgien, Frankreich, Deutschland, Spanien, VK) und an einigen Universitäten anderer Länder (z.B. Wien) sind diese Konzepte bereits Pflichtbestandteile des Medizinstudiums [76].

\subsection{Klinische Qualitätsstandards und Kompetenzen} des Facharztes für Physikalische und Rehabilitative Medizin Für Menschen mit Behinderungen ist nicht nur der gleichberechtigte Zugang zur Rehabilitation von elementarer Bedeutung, vielmehr muss die Rehabilitation auch auf kompetente und sachgerechte Weise durchgeführt werden. Um dies sicherzustellen ist ein regelmäßiges Feedback der Patienten notwendig.

Im Zuge ihrer Ausbildung eignen sich PRM-Fachärzte zahlreiche Fertigkeiten an. Aufgrund ihrer medizinischen Grundausbildung sind sie bereits mit bestimmten Grundkenntnissen ausgestattet, die sie allerdings durch die Aneignung von zusätzlichem Wissen und von Erfahrung im Rahmen ihrer gemeinsamen Ausbildung in innerer Medizin, Chirurgie, Psychiatrie usw. ausbauen können. Die Kernkompetenzen der PRM erhalten die PRM-Fachärzte im Zuge ihrer Facharztweiterbildung. Dieses Wissen kann im Zuge der Arbeit in speziellen Teilbereichen des Fachgebietes weiter vertieft werden (Abb.3). In einigen europäischen Ländern werden derzeit die Kriterien in Teilbereichen den Fachgebietes erarbeitet.

Diese Kompetenzen umfassen:

- medizinische Untersuchungen zur Ermittlung der zugrunde liegenden Diagnose;

- Beurteilung der funktionellen Leistungsfähigkeit und des Veränderungspotenzials;

- Beurteilung der Aktivitäten und der Teilhabe sowie Kontextfaktoren (persönliche Faktoren und Umwelt, siehe Abschnitt 3.2);

- Kenntnis der Grundprinzipien von Rehabilitationsprozessen und deren wissenschaftlichen Basis (siehe Abschnitte 4.1 und 4.4)

- das Erstellen eines Rehabilitationsplans (siehe Abschnitt 5.5);

- Kenntnis medikamentöser und physikalischer Therapien (einschließlich physikalischer Modalitäten, Naturheilverfahren und anderen, siehe Abschnitt 5.6), Erfahrung mit diesen Behandlungen und Anwendung derselben;

- Evaluieren und Messen der Rehabilitationsergebnisse;

- Vorbeugung und Behandlung von Komplikationen;

- Erstellen von Prognosen zur Erkrankung/zum Zustand und zu den Ergebnissen der Rehabilitation;

- Kenntnis der Rehabilitationstechnologie (Orthotik, Prothetik, technische Hilfsmittel usw.);

- Teamarbeit und Teamleitung;

- pädagogische Fähigkeiten (gegenüber den Patienten, dem Pflegepersonal, den Teammitgliedern und anderen);

- Kenntnis des Sozialsystems und der Gesetzgebung hinsichtlich Behinderung;

- Grundkenntnisse wirtschaftlicher (und finanzieller) Aspekte der Rehabilitation.

PRM-Fachärzte bedürfen regelmäßig noch weiterer Grundeigenschaften. Sie müssen kommunikationsfreudig und geschickt im Umgang mit anderen Menschen sein; auch müssen sie die Fähigkeit besitzen, die Patienten und deren Familien aufzuklären und 


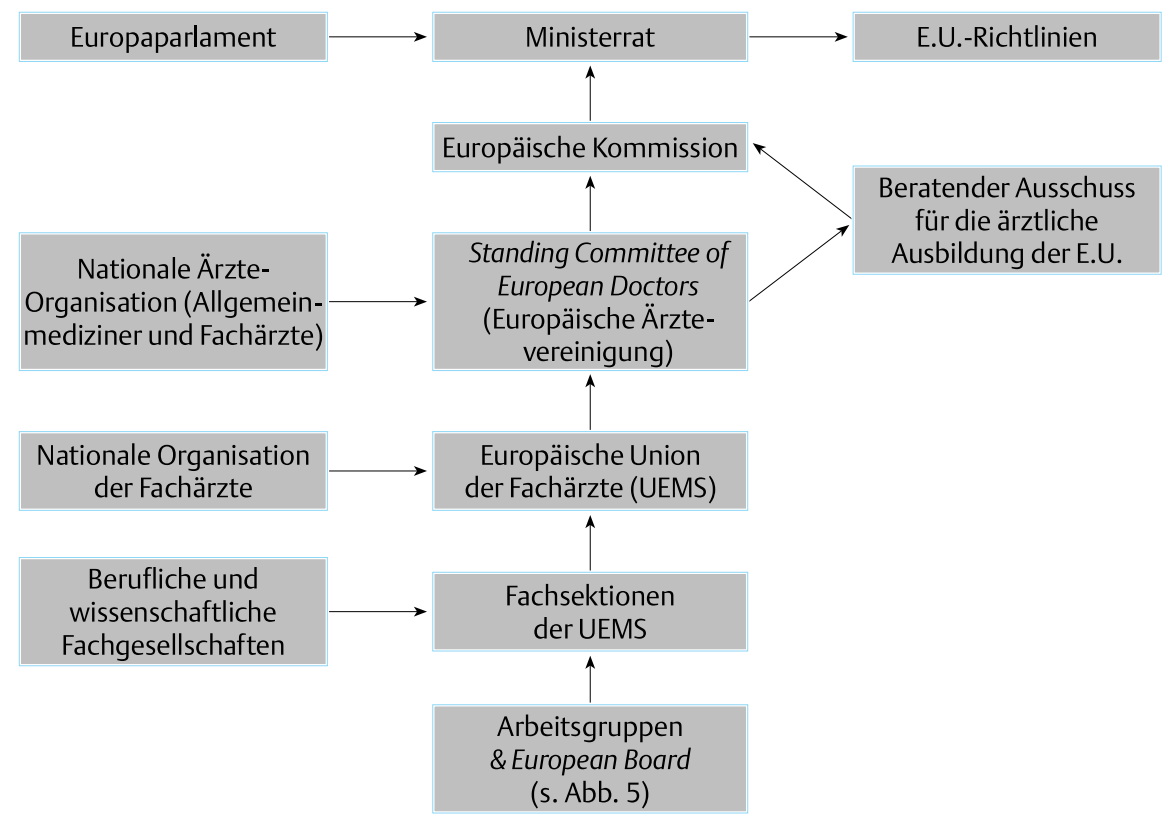

Abb. 4 Medizinische Organisationen der Europäischen Union.

zu führen. Sie müssen sich Strategien aneignen, mit denen sie den Patienten helfen können, ihre eigenen Bewältigungsfähigkeiten zu entwickeln. Ihre Tätigkeit umfasst auch die Zusammenarbeit mit behördlichen und anderen Stellen (Gesundheitsund Sozialdienste sowie Selbsthilfegruppen), um für ihre Patienten die bestmöglichen Lösungen zu finden. Sie bringen sich auch bei der Entwicklung von Hilfsdiensten für Menschen mit Behinderungen mit ein, wofür sie ebenfalls einschlägige Kenntnisse besitzen müssen.

\subsection{Kontinuierliche berufliche Ausbildung (Continuing Professional Development, CPD) und medizinische Weiterbildung (Continuing Medical Education, CME)}

CPD und CME sind integraler Bestandteil der beruflichen Praxis eines jeden Facharztes, und PRM-Fachärzte müssen, wie alle anderen Ärzte, ihre Kompetenz fortwährend weiterentwickeln und nachweisen. CPD umfasst alle Aspekte der beruflichen Weiterbildung praktizierende Ärzte, CME ist ein Bestandteil davon. Für das Fachgebiet gibt es europaweit Lernprogramme zur Fortbildung von PRM-Fachärzten und deren Mitarbeiter in Rehabilitationsteams. Die Lernprogramme beinhalten wissenschaftliche Grundlagen, klinische Lerninhalte sowie Untersuchungstechniken und technische Themen.

Auf europäischer Ebene wurde ein CME- und CPD-Programm zur Akkreditierung internationaler PRM-Kongresse und -Veranstaltungen organisiert. Das Programm beruht auf einer Vereinbarung, die zwischen dem Europäischen Akkreditierungsrat für CME (EACCME) der UEMS, der UEMS-PRM-Sektion und dem Board geschlossen wurde (Kapitel 6.4). Für alle Fachgebiete gelten in Europa die gleichen Bestimmungen. Das European Board for PRM hat einen CPD-/CME-Ausschuss eingesetzt, der für die einschlägigen Fortbildungsprogramme innerhalb des Fachgebietes, die Approbation einiger wissenschaftlicher Veranstaltungen auf europäischer Basis und den wissenschaftlichen Status der vom Board zertifizierten PRM-Fachärzte verantwortlich ist.
Der EACCME zeichnet für die Koordination dieser Tätigkeiten für alle medizinischen Fachgebiete verantwortlich. Auf der UEMSWebsite befinden sich Details über die Anforderungen an eine medizinische Weiterbildung für alle Fachärzte in Europa (www.uems.org). Jeder vom Board anerkannte PRM-Facharzt muss im Laufe von 5 Jahren zur Neuüberprüfung und -zulassung 250 Ausbildungspunkte erreichen. Die Ärzte müssen ihre CMEAnforderungen erfüllen, bevor sie zugelassen werden können, was demnächst einen wesentlichen Teil der innerstaatlichen und europäischen Anforderungen bilden wird. In einigen Ländern wurden obligatorische CPD-/CME-Programme eingeführt, die in der medizinischen Praxis ständig an Bedeutung gewinnen. Die Regeln dazu befinden sich in Anhang 6.

Das erste vom European Board unterstützte Veranstaltung ist die im Jahr 2000 gegründete European School in Marseille on Posture and Movement Analysis. Dabei handelt es sich um einen jährlichen zweiwöchigen Ausbildungskurs für Ärzte, Ingenieure und andere Rehabilitationsfachleute aus ganz Europa. Zur Zeit werden weitere Kurse nach den Richtlinien des Board vorbereitet.

\subsection{Sektion Physikalische und Rehabilitative Medizin der Europäischen Union der Fachärzte (UEMS)}

\subsubsection{Geschichte}

Die 1958 gegründete UEMS ist die einzige öffentlich-rechtliche medizinische Körperschaft in der Europäischen Union mit Verantwortung für ärztliche Fachgebiete. Die Vereinigung besteht aus einzelnen Sektionen für jedes Fachgebiet in Europa, wobei die Physikalische und Rehabilitative Medizin eines der ersten Fachgebiete war, die als eigenständige Disziplinen anerkannt wurden. 1971 wurde die PRM-Sektion und 1991 das European Board of PRM als Teil der PRM-Sektion gegründet. Dieses hat in besonderem Maße die Basis für die Weiterbildung und kontinuierliche Fortbildung geschaffen. Als öffentlich-rechtliche Körperschaft der Europäischen Kommission ist die UEMS gegenüber dem Standing Committee of Doctors verantwortlich, zu dem die einzelstaatlichen medizinischen Organisationen ihren Beitrag leisten. Sobald eine Fachdisziplin in zwei Dritteln des UEMS-Mitgliedsstaates anerkannt ist, kann sie eine eigene Fachsektion 


\begin{tabular}{|c|c|c|}
\hline & $\begin{array}{l}\text { Executive Committee } \\
\text { Präsident, Schrifttührer, Schatzmeister, } \\
\text { stellvertretender Schrifttührer, Vorsitzende } \\
\text { der Unterausschüsse, Ausschussmitglied }\end{array}$ & $\begin{array}{l}\text { er, } \\
\text { nde } \\
\text { ed }\end{array}$ \\
\hline$\downarrow$ & $\downarrow$ & $\downarrow$ \\
\hline PRM Board & $\begin{array}{l}\text { Ausschuss für klinische } \\
\text { Angelegenheiten }\end{array}$ & $\begin{array}{l}\text { Ausschuss für die } \\
\text { berufliche Praxis }\end{array}$ \\
\hline$\downarrow$ & $\downarrow$ & $\downarrow$ \\
\hline $\begin{array}{l}\text { Ausbildung } \\
\text { · Prüfung } \\
\text { - Kontrollbesuche der } \\
\text { Ausbildungsstätten } \\
\text { Ausbildung } \\
\text { - Medizinische } \\
\text { Weiterbildung (CME) } \\
\text { - Kongresse } \\
\text { - Austauschprojekte } \\
\text { Werbung \& Sponsoring }\end{array}$ & $\begin{array}{l}\text { Klinische Standards } \\
\text { Klinische Fragen zur } \\
\text { Tätigkeit des Fachgebiets } \\
\text { und der Teilbereiche des } \\
\text { Fachgebietes } \\
\text { Forschung:-Verbindungen zu: } \\
\text { - Eur. Gesellschaft für PRM } \\
\text { - Académie Européenne }\end{array}$ & $\begin{array}{l}\text { Inhalt des Fachgebiets der PMR } \\
\text { - Universität/Abteilung/ } \\
\text { Private Praxis } \\
\text { Neue Abteilungs- und } \\
\text { akademische Posten } \\
\text { - Definition der akademischen } \\
\text { Posten \& Klärung zwischen } \\
\text { den Mitgliedsstaaten } \\
\text { - Karrieremöglichkeiten } \\
\text { Demografie } \\
\text { - Beratender Führungsausschuss } \\
\text { über Beziehungen zu anderen } \\
\text { Fachgebieten und Berufen } \\
\text { - UEMS/ACMT, E.U., W.H.O. }\end{array}$ \\
\hline
\end{tabular}

Präsident, Schriftführer, Schatzmeister, ellvertretender Schriftführer, Vorsitzende der Unterausschüsse, Ausschussmitglied

bilden: In jedem der 28 Mitgliedsstaaten des erweiterten Europas ist PRM als eigenständiges Fachgebiet anerkannt, wobei die neuen Mitglieder die gleichen Grundsätze befolgen. Die PRM ist damit eine der wenigen, in ganz Europa anerkannten Disziplinen und im Council der UEMS vertreten.

\subsubsection{Struktur und Aufgaben der Organisation}

In Europa ist das Fachgebiet über die PRM-Sektion der UEMS organisiert (Abb.4). Es handelt sich dabei um eine öffentlich-rechtliche Körperschaft, die gegenüber dem Europäischen Kommissar für Gesundheit verantwortlich ist und die als einzige medizinische Körperschaft von der Europäischen Union anerkannt ist. Ziel der Sektion ist die Förderung des Fachgebiets und seine Harmonisierung auf europäischer Ebene, und zwar durch die Facharztausbildung und die kontinuierliche Weiterbildung mit Rezertifizierung und -akkreditierung. Sie arbeitet an der Weiterentwicklung klinischer Standards für die Praxis und unterstützt die Durchführung der für die Weiterentwicklung des Gebiets notwendigen Forschungen.

Die Sektion für PRM ist (zusammen mit anderen Fachgebieten) gegenüber der UEMS verantwortlich und hat eine enge Zusammenarbeit mit der Europäischen Kommission und dem Europarat begonnen. Mit zahlreichen anderen Organen wie z. B. der International Society for Physical Medicine and Rehabilitation, der American Academy of PRM sowie der Bone and Joint Decade hat sie enge Verbindungen geknüpft. Unter einem Executive Committee sind drei Hauptausschüsse eingerichtet, deren Aufgaben in Abb. 5 dargestellt sind. Die Sektion arbeitet eng mit anderen europäischen PRM-Körperschaften wie z.B. der Académie Européenne de Médecine de Réadaptation und der European Society of PRM zusammen.
Die PRM-Sektion der UEMS hat folgende Ausschüsse

- Ausschuss für theoretische und praktische Ausbildung (Training and Education Committee) - ein rechtlich eigenständiger Ausschuss - das European Board of PRM.

- Ausschuss für klinische Angelegenheiten (Clinical Affairs Committee).

- Ausschuss für die berufliche Praxis (Professional Practice Committee).

\subsubsection{Mitglieder}

Mit dem Beitritt von zehn neuen Mitgliedsstaaten zur Europäischen Union am 1. Mai 2004 zählt die UEMS nunmehr 28 Vollmitglieder: die EU-Länder, die Schweiz, Norwegen und Island. Rumänien, Bulgarien, Kroatien und die Türkei haben Beobachterstatus. In diesen Ländern arbeiten 10280 ausgebildete Fachärzte; 2800 befinden sich in Ausbildung [76]. Eine wichtige Aufgabe der UEMS ist es daher, ein wirkungsvolles Verbindungsglied zwischen diesen Ländern auf europäischer Ebene zu bilden. Die Anzahl der Fachärzte für Physikalische und Rehabilitative Medizin ist in Europa sehr unterschiedlich; die demografischen Einzelheiten gehen aus Anhang 2 hervor.

Trotz der Unterschiede, die in den Gesundheitssystemen der einzelnen Länder Europas bestehen, sind die Einrichtungen für Physikalische und Rehabilitative Medizin im Prinzip ähnlich aufgebaut. Im Rahmen des weiteren Harmonisierungsprozesses werden in praxisbasierten Arbeitsgruppen Vorschläge für klinische Standards erarbeitet.

\subsubsection{Ausbildung in PRM}

Die Facharztausbildung wird in Abschnitt 6.4.1 beschrieben. In den einzelnen Ländern gibt es geringfügige Unterschiede hinsichtlich des Zugangs zur Fachausbildung. Trotz dieser Unterschiede ist der Lehrplan im gesamten Kontinent sehr ähnlich aufgebaut und entspricht auch jenem des American Board. Dem European Board of PRM obliegt die Aufgabe der Harmonisierung 
der Fachausbildung in ganz Europa, was folgende Aspekte umfasst [74].

- Europäische Facharztprüfung (European Board Certification);

- Kontinuierliche berufliche Weiterentwicklung und medizinische Weiterbildung mit einer Rezertifizierung und -akkreditirung in Abständen von 10 Jahren;

- Anerkennung europäischer Ausbildner und Weiterbildungsstätten auf der Basis sog. Site visits.

Gemeinsames Ziel dieser Harmonisierungsbemühungen ist es, Fachärzte aus- und weiterzubilden, die in allen europäischen Gesundheitssystemen arbeiten können, und den nationalen medizinischen Behörden/Arbeitgebern zu ermöglichen, die Kenntnisse und das Expertenwissen der in einem anderen europäischen Land ausgebildeten Fachärzte anzuerkennen. Auf der Website der Sektion www.euro-prm.org finden sich alle Einzelheiten über die Sektion und das Board, einschließlich des Curriculums für das Fachgebiet (Anhang 5).

\subsection{Académie Européenne de Médecine de Réadaptation}

Diese aus 50 erfahrenen Fachärzten aus ganz Europa zusammengesetzte Körperschaft wurde 1969 gegründet. Zum Beitritt werden anerkannte Wissenschaftler eingeladen, die einen wesentlichen Beitrag zum Fachgebiet, insbesondere zu seinen humanitären Aspekten geleistet haben. Zweck der Académie ist die Weiterentwicklung aller Bereiche der Rehabilitation zum Wohle jener, die ihrer bedürfen. In diesem Sinne fördert sie die Ausbildung und Forschung in ganz Europa und dient als Bezugspunkt für alle wissenschaftlichen Fragen sowie Ausbildungs- und Forschungsangelegenheiten. Sie treibt den Gedanken- und Informationsaustausch voran und kümmert sich um einen reibungslosen Austausch von PRM-Fachärzten zwischen den einzelnen Ländern. Darüber hinaus beteiligt sich die Académie auch an der Diskussion um ethische und moralishe Fragen.

Die Devise der Académie lautet: „Societas vir origo ac finis“, was soviel bedeutet wie „Der Mensch ist sowohl der Ursprung als auch das Ziel der Gesellschaft“.

Die Académie arbeitet völlig unabhängig u.a. im Bereich der Veröffentlichung von Fachbüchern und in der Unterstützung von Ausbildung und Forschung. Eine Reihe von Monografien zum aktuellen Stand des Fachgebietes (state of the art) werden von der Académie herausgegeben, u.a.:

- La Plasticité de la Fonction Motrice. herausgegeben von J.-P. Didier (Springer 2004);

- Assessment in Physical Medicine and Rehabilitation. herausgegeben von M. Barat und F. Franchignoni (Maugeri Foundation Books 2004);

- Vocational Rehabilitation. herausgegeben von C. Gobelet und F. Franchignoni (Springer 2006);

- Les Fonctions Sphinctériennes. herausgegeben von A. Chantraine (Springer 2006).

Weitere Veröffentlichungen sollen demnächst erscheinen, wie z. B. im Bereich der onkologischen und der Schmerzrehabilitation.
Die Académie diskutiert über ethische Fragen und fungiert diesbezüglich als „Wächter“ über die Perspektiven des Fachgebiets. Eine der jüngsten Debatten zum Thema Gewalt und Behinderung wurde zusammengefasst und als kurzer Beitrag mit dem Titel „Violence and Handicap“ [77] veröffentlicht.

Zahlreiche Studiengänge werden von der Académie unterstützt, wie z. B. die European School on Posture and Movement Analysis (Europäische Schule zur Analyse der Haltung und Bewegung) an der Universität von Marseille mit Erasmus-Finanzierung. Zur Nachwuchsförderung in der Forschung auf dem Gebiet der PRM, vergibt die Académie jährlich einen Preis, der auch von der Schweizerischen Gesellschaft für Paraplegie unterstützt wird.

\subsection{Europäische Gesellschaft für Physikalische und Rehabilitative Medizin (ESPRM)}

Die Europäische Gesellschaft für PRM wurde 2003 gegründet und befasst sich mit der Forschung und Ausbildung in PRM in Europa. Es handelt sich dabei um die Nachfolgegesellschaft der 1963 gegründeten Föderation für Physikalische Medizin und Rehabilitation (European Federation of Physicial Medicine and Rehabilitation). Ziel dieser Gesellschaft ist die Koordinierung der Forschungsaktivitäten in Europa und die Förderung des wissenschaftlichen Austauschs. Die Gesellschaft bietet allen entsprechend qualifizierten PRM-Fachärzten und Mitgliedern der PRMGesellschaften der einzelnen Länder Europas eine individuelle Mitgliedschaft an. Die individuelle Mitgliedschaft ist kostenlos. Die ESPRM arbeitet derzeit an der Erstellung einer interaktiven elektronischen Plattform (www.esprm.org), über welche Informationen zu Forschungsprojekten, Stipendien und Finanzierungsmöglichkeiten zu finden sind; zudem bietet sie laufend aktualisierte Informationen über Studiengänge, Kongresse, Stipendien u.a.

Im 2-Jahres-Rhythmus werden von der ESPRM wissenschaftliche Kongresse zum Fachgebiet abgehalten. Die Hauptthemen der letzten drei Kongresse lauteten:

- State of the Art in der Rehabilitationsmedizin - Klinische Standards, Messung von Ergebnissen und wirksame Interventionen in der neurologischen Rehabilitation, Rehabilitation des Bewegungsapparates und Rehabilitation nach Amputation (13. Europäischer Kongress für Rehabilitation, Brighton, VK, 2002);

- „Fortschritte in PRM - Traditionelle und moderne Konzepte“ (14. Europäischer Kongress für Rehabilitation, Wien, Österreich, 2004);

- Evidenzbasierte Rehabilitation, Physikalische Medizin und Rehabilitation nach Lungentransplantation und bei Diabetes mellitus (15. Europäischer Kongress für Rehabilitation, Madrid, Spanien, 2006).

Für künftige Europäische Kongresse für PRM wurden durch die ESPRM Standards hinsichtlich Organisation und Ablauf erarbeitet. Demzufolge werden die Kongresse nach einem standardisierten Programm ablaufen, wobei für folgende Themenbereiche jeweils ein halber Tag vorgesehen wird:

- Funktionsbiologie (Grundlagenwissenschaften);

- Funktionstechnologie (Rehabilitationstechnologie);

- Klinische Wissenschaften (PRM bei bestimmten Erkrankungen); 
- Wissenschaften der menschlichen Funktionsfähigkeit (einschließlich Epidemiologie, soziale Funktionen und verwandte Themen).

Die Dynamik der PRM ist nicht von Regierungen, Versicherungsgesellschaften, Politikern noch sonstigen Berufsgruppen abhängig, sondern allein von den Aktivitäten derjenigen, die sie praktizieren.

\section{Forschung auf dem Gebiet der Physikalischen und Rehabilitativen Medizin}

\subsection{Bedeutung der Forschung zur Ermittlung des Rehabilita- tionsbedarfs und Wert jetziger und zukünftiger Ansätze}

Die PRM befolgt die Grundsätze der evidenzbasierten Medizin ohne Einschränkungen. Die Forschung auf dem Gebiet der PRM konnte in den letzten 20 Jahren große Fortschritte verzeichnen. Während traditionell die physiologischen Wirkmechanismen der physikalischen Funktionsmodalitäten im Laufe der letzten 15 Jahre im Zentrum des wissenschaftlichen Interesses lagen, werden zunehmend auch prospektive Studien durchgeführt, in denen die klinische Wirksamkeit der Rehabilitation bei zahlreichen Erkrankungen wie z. B. Kreuzschmerzen, Schlaganfall, Hirnund Rückenmarksverletzung [78-80], rheumatoider Arthritis sowie Herz-Kreislauf-, Lungen- und Stoffwechselstörungen untersucht werden. Für bestimmte Erkrankungen stehen auch bereits Metaanalysen von kontrollierten Studien zur Verfügung.

\subsubsection{Bedeutung der Forschung}

Das Fachgebiet PRM hat sich zum Ziel gesetzt, das Interesse und das Engagement für die Forschung auf dem Gebiet der Rehabilitation zu steigern. Ihre Fachvertreter sind überzeugt, dass Forschung notwendig ist, um die grundlegenden Prozesse der Rehabilitation zu verstehen. So muss untersucht werden, wie der Mensch neue Fertigkeiten erwerben kann und wie sich die verschiedenen Gewebe des Körpers (z.B. Muskeln oder neuronale Bahnen im ZNS) von einem Trauma oder einer Krankheit erholen oder sich an deren Auswirkungen anpassen können. Forschung dient auch dazu, die Inzidenz und Prävalenz von Behinderungen zu ermitteln und die Faktoren zu identifizieren, die für eine Wiederherstellung und für die Fähigkeit zur Veränderung, zum Erwerben neuer Fertigkeiten und zum Ansprechen auf die Rehabilitation entscheidend sind.

Neue Technologien sollten so adaptiert werden, dass sie auch durch Menschen mit Behinderungen genutzt werden können. Die Rehabilitationstechnologie ist eine der bedeutendsten und viel versprechendsten Forschungsbereiche und wird dies auch in Zukunft sein. Tissue engineering - Gewebezüchtung - und sonstige moderne Technologien leisten ihren Beitrag auf diesem Gebiet. Die Kosten für die medizinische Versorgung und die Rehabilitation werden weiter steigen, und die Politiker werden die Anbieter von Dienstleistungen der medizinischen Versorgung zwingen, ihre Ausgaben einzuschränken und zu beweisen, dass sie diese Versorgung effizient erbringen. In der Diskussion mit Patienten, Politikern, Gesundheitsministerien und Versicherungsgesellschaften ist die PRM eine verlässliche Partnerin, weil sie in der Lage ist, ihre öffentlich vorgebrachten Argumente durch fundierte Daten zu untermauern, was nur durch Forschung erreicht wrden kann.

\subsubsection{Herausforderungen an die Forschung auf dem Gebiet der Physikalischen und Rehabilitativen Medizin}

Die Rehabilitationsforschung begnügt sich weder bei der Grundlagenforschung noch bei der Forschung im Bereich der medizinischen Praxis mit den üblichen Ansätzen. So konnten bezüglich der Messmethoden beträchtliche Fortschritte erzielt werden. Nur hierdurch wurden in vielen Bereichen randomisierte kontrollierte Studien ermöglicht. Forschungsarbeiten, die sich auf klinische Endpunkte beziehen sind jedoch weniger aussagekräftig, wenn sich die verfolgten Rehabilitationsziele bei den einzelnen Personen innerhalb der Untersuchungsgruppe unterscheiden, insbesondere wenn die Unterschiede nicht auf biologische, sondern vielmehr auf persönliche oder soziale Faktoren zurückzuführen sind. Die im Bereich der klinischen Psychologie entwickelten Designs klinischer Studien sind in der Rehabilitation oftmals zielführender und wissenschaftlich angemessener als jene, die zur Untersuchung der Wirksamkeit von Arzneimitteln erstellt werden. Eine wissenschaftlich begründete Analyse der Wirksamkeit der Rehabilitation kann vielfach nur mit einer Kombination aus qualitativen und quantitativen Untersuchungsmethoden erreicht werden.

Staatliche Stellen und Anbieter von Rehabilitationsdiensten erwarten in der Regel einen Nachweis der Kosteneffizienz von Rehabilitationsmaßnahmen. Sie verlangen darüber hinaus, dass die Dienstleistung als Ganzes evaluiert wird, weil dem behandelnden Team eine breite Palette unterschiedlicher Techniken zur Verfügung stehen muss, um den unterschiedlichen Bedürfnissen der einzelnen Mitglieder einer Patientengruppe gerecht werden zu können.

Hier liegt der Kernpunkt des Problems, weil in der PRM-Praxis die Ergebnisse durch eine Reihe von Interventionen bzw. durch die Kombination von Interventionen erreicht werden. Der Nachweises der Wirkung einer einzelnen Rehabilitationsintervention entspricht nicht dem „wirklichen Leben“. Sie ist zwar wichtig um einzelne wirksame Verfahren zu ermitteln, die in ein Programm aufgenommen werden sollen; für eine zielführende Beurteilung dieses Programms als Ganzes ist sie dennoch nicht geeignet. Derzeit gibt es eine Reihe von Initiativen, die sich auf diese technischen und wissenschaftlichen Probleme konzentrieren und eine regelmäßige Überwachung der Effektivität und des Kosten-Nutzen-Verhältnisses in der Rehabilitationspraxis ermöglichen werden.

\subsection{Forschungseinrichtungen und -ressourcen}

Der wichtigste Schritt zur Steigerung des Umfangs und Niveaus der Forschungsarbeiten auf dem Gebiet der PRM ist die Organisation einer Kommunikationsplattform für alle Beteiligten an relevanten Forschungsaktivitäten in Europa. Es müssen Informationen über künftige oder laufende Projekte, Finanzierungsmöglichkeiten und Stipendien, Protokolle, Fragebögen, Messmethoden und Austauschprogramme zur Verfügung stehen, und diese müssen für Kliniker, Forscher und Manager leicht zugänglich sein. Um die dringenden Fragen der Effektivität von Rehabilitationsmaßnahmen beantworten zu können, werden beträchtliche Mittel zur Finanzierung der Forschung benötigt.

Um diese einschlägigen Fragen beantworten zu können, müssen die Forschungsmittel für PRM und die Präsenz des Fachgebiets 
an den Universitäten deutlich erhöht werden. Sowohl auf europäischer als auch auf nationaler Ebene muss die Forschungsplanung und die Kooperation mit anderen Forschungsgebieten vorangetrieben werden. Dies wird dazu beitragen, die Auswirkungen von Behinderungen auf persönlicher und gesellschaftlicher Ebene zu verringern.

\subsection{Ausbildung in der Forschung}

Wichtig ist, dass alle in der Rehabilitation Tätigen die oben genannten fachlichen Anforderungen an die Rehabilitationsforschung verstehen. Da viele Aspekte der Rehabilitation ein multidisziplinäres Vorgehen erfordern, sind es auch die multidisziplinären Forschungsgruppen und Abteilungen, die die fruchtbringendste Basis für die Ausbildung in den verschiedenen Berufen der Rehabilitation, einschließlich der ärztlichen Tätigkeit, bilden. Es ist nicht sinnvoll, wenn die Kliniker einerseits die Rehabilitation ganzheitlich und multidisziplinär umsetzen und andererseits die Forschung in einzelnen, auf ihr eigenes Fachgebiet beschränkten Forschungsteams betreiben, die zudem völlig getrennt voneinander arbeiten. Es werden große Anstrengungen unternommen, um auch die in Weiterbildung zum Facharzt PRM befindlichen Ärzte in Kontakt mit solchen multidisziplinären Forschungsteams $\mathrm{zu}$ bringen und sie davon profitieren $\mathrm{zu}$ lassen. Auch wenn in vielen Ländern mit den verfügbaren Ressourcen nur die Ausbildungserfordernisse der Talentiertesten erfüllt werden können, so bessert sich die Situation doch schrittweise. Der Anteil an Weiterbildungskandidaten, die zusätzlich zu ihrer beruflichen Qualifikation ein Doktorat (PhD) o. Ä. aufweisen, nimmt zu und wird das Fundament für die weitere Forschung und akademische Arbeit bilden.

\subsection{Veröffentlichung der Forschungsergebnisse}

In jedem Land werden die Ergebnisse der wissenschaftlichen Forschung auf dem Gebiet der PRM in einschlägigen Fachzeitschriften veröffentlicht. Auf europäischer Ebene werden Berichte über multidisziplinäre Forschungsprojekte in den Zeitschriften „Journal of Rehabilitation Medicine“, „Disability and Rehabilitation“, „Clinical Rehabilitation“ und „Europa Medicophysica“ veröffentlicht. Auf weltweiter Ebene erhält zur Zeit eine Veröffentlichung in den Archives of Physical Medicine and Rehabilitation den höchsten „Impact Factor“.

\section{Zukünftige Entwicklungen}

\subsection{Grundsätze}

Sowohl in den Industrie- als auch in den Entwicklungsländern steigt die Lebenserwartung dramatisch an. Noch dramatischer ist aber, dass der Bedarf an Rehabilitationsleistungen aufgrund der verbesserten Überlebenschancen nach Unfällen und Krankheiten, aber auch durch das zunehmende Alter der Bevölkerung in allen europäischen Ländern zunehmen wird [81]. Gleichzeitig steigen die Ansprüche der Menschen in Europa an die Lebensqualität kontinuierlichen an [81]. Die Rehabilitationssysteme müssen aus diesem Grund ständig weiterentwickelt werden, wobei folgende Grundsätze zu berücksichtigen sind:

- Die Rehabilitation nach Unfällen oder akuten Krankheitsereignissen sowie bei chronischen Leiden stellt ein grundlegendes Menschenrecht dar [82].
- Die gesamte Bevölkerung Europas hat ein Anrecht auf einen gleichberechtigten und einfachen Zugang zu allen Bereichen der Rehabilitation, einschließlich der spezialisierten Rehabilitationsmedizin und der assistierenden Technologien, sowie auf soziale Unterstützung.

- Es ist ein gleichmäßig hoher Versorgungsstandard in der Rehabilitation in ganz Europa anzustreben, der auch eine Qualitätssicherung und auf wissenschaftlichen Erkenntnissen beruhende Behandlungen einschließt.

- Es muss eine wissenschaftliche Basis für die Entwicklung von Rehabilitationsmodellen und Versorgungsstandards als Leitlinie für die klinische Praxis entwickelt werden.

\subsection{Ziele}

Um diese Ziele in der PRM erreichen zu können, müssen Maßnahmen ergriffen werden,

- um das allgemeine Verständnis und das Bewusstsein für die Bedürfnisse von Menschen mit Behinderungen zu verbessern;

- um die Vorteile der Rehabilitation bekannt zu machen, sodass eine Kultur entsteht, in der der Zugang zur adäquaten Rehabilitation als grundlegendes Menschenrecht angesehen wird;

- um das Verständnis und die Kooperation zwischen Nichtregierungsorganisationen für Menschen mit Behinderungen und dem Fachgebiet der PRM zu vertiefen;

- um umfassende Rehabilitationseinrichtungen in ganz Europa zu schaffen, die über spezialisierte und gut ausgebildete Rehabilitationsteams und ausreichend ausgestattete Rehabilitationseinrichtungen verfügen. Zusätzlich dazu sollten auf Gemeindeebene Rehabilitationsstrukturen für das Management chronischer zu Behinderungen führender Erkrankungen eingerichtet werden;

- um Systeme aufzubauen, mit denen sichergestellt werden kann, dass in allen Ländern Europas für das Fachgebiet Physikalische und Rehabilitative Medizin genügend kompetente Fachärzte zur Verfügung stehen;

- um einheitlich hohe Versorgungsstandards auf der Basis aktueller Kenntnisse einzuführen. Diese sollten auch eine Qualitätskontrolle und den Zugang zu unterstützender Technologie beinhalten;

- um neue technische Entwicklungen in die PRM-Praxis umzusetzen. Dies hilft der Rehabilitation in hohem Maß dabei, bessere Ergebnisse zu erzielen: Die Technologie sollte zunehmend dazu beitragen, dass Menschen mit Behinderungen ein unabhängiges Leben mit guter Lebensqualität führen können;

- um wissenschaftliche Tätigkeiten und Forschung auf dem Gebiet der Rehabilitation zu fördern und eine angemessene Finanzierung sicherzustellen, damit bessere Ergebnisse für Personen mit Behinderungen erzielt werden können;

- um ein Umfeld zu schaffen, in der Menschen mit Behinderungen in vollem Umfang an der Gesellschaft teilhaben können.

Die PRM-Fachärzte werden zum Erreichen dieser Ziele eng mit Personen mit Behinderungen und ihren Organisationen zusammenarbeiten.

Alle diese Maßnahmen werden es Personen mit Behinderungen ermöglichen, zur Gesellschaft und zur Gemeinschaft in Europa einen wesentlichen Beitrag zu leisten. 
${ }^{1}$ European Academy of Rehabilitation Medicine, European Federation of Physical and Rehabilitation Medicine, European Union of Medical Specialists (Physical and Rehabilitation Medicine Section): White Book on Physical and Rehabilitation Medicine. Universidad Complutense de Madrid; 1989

2 Martin J, Meltzer H, Eliot D. Report 1; The Prevalence of Disability among Adults. Office of Population, Census and Surveys, Social Survey Division. OPCS Surveys of Disability in Great Britain, 1988-89. London: HMSO; 1988

${ }^{3}$ Bax MCO, Smythe DPL, Thomas AP. Health care for physically handicapped young adults. British Medical Journal 1988; 296: 1153 - 1155

${ }^{4}$ UEMS-PRM-Section: Definition of Physical and Rehabilitation Medicine. www.euro-prm.org. 2005

${ }^{5}$ Ward $\mathrm{AB}$, Chamberlain MA. Disabled Young Adults. In: Evans CD, Goodwill J, Chamberlain MA (eds): Rehabilitation of the Physically Disabled Adult, $2^{\text {nd }}$ Ed. London: Chapman \& Hall; 1996

${ }^{6}$ Dennis M. Langhorne P. So stroke units save lives: where do we go from here? British Medical Journal 1994; 309: 1273-1277

${ }^{7}$ Beyer HM, Beyer L, Ewert T, Gadomski M, Gutenbrunner C, Kröling P, Pages HI, Sidel E, Smolenski U, Stucki G: Weißbuch Physikalische Medizin und Rehabilitation. Phys Med Rehab Kuror 2002; 12: M1 - M30

${ }^{8}$ Deutscher Bundestag (2004): Unterrichtung durch die Bundesregierung: Bericht der Bundesregierung über die Lage behinderter Menschen und die Entwicklung ihrer Teilhabe. Drucksache 15/4575: Bundesdruckerei, Berlin, 2005; 146

9 Rice-Oxley M, Turner-Stokes L: Effectiveness of brain injury rehabilitation. Clinical Rehabilitation 1999; 13 (Suppl 1): 7-24

${ }^{10}$ Redmond AD, Johnstone S, Maryosh J, Templeton J. A trauma centre in the UK. Annals of the Royal College of Surgeons of England 1993; 75(5): $3173-3200$

${ }^{11}$ Templeton, J. Organising the management of life-threatening injuries. Journal of Bone \& Joint Surgery - British Volume 1994; 76 (1): $3-5$

12 Kaste M, Skyhoj Olsen T, Orgogozo J, Bogousslavsky J, Hacke W. Organization of stroke care: education, stroke units and rehabilitation. European Stroke Initiative (EUSI). Cerebrovascular Diseases 2000; 10 (Suppl 3): 1-11

${ }^{13}$ Anonymous. Organised inpatient (stroke unit) care for stroke. Stroke Unit Trialists' Collaboration. [update in Cochrane Database of Systematic Reviews]. Cochrane Database of Systematic Reviews, 2002

${ }^{14}$ National Clinical Guidelines for Stroke, $2^{\text {nd }}$ Edition. Clinical Effectiveness and Evaluation Unit, Royal College of Physicians. London: Royal College of Physicians of London, 2004

15 Turner-Stokes L, Nyein K, Halliwell D. The Northwick Park care needs assessment (NPCNA): a directly costable outcome meaure in rehabilitation. Clinical Rehabilitation 1999; 13: $253-267$

${ }^{16}$ Livingston MG. Journal of Neurology, Neurosurgery \& Psychiatry 1985; 48: 876-881

17 British Society of Rehabilitation Medicine Working Party Report on Traumatic Brain Injury. London: British Society of Rehabilitation Medicine; 1998

18 McLellan DL. Rehabilitation. British Medical Journal 1991; 303: 355 357

${ }^{19}$ Bent N, Tennant A, Swift T, Posnett J, Chamberlain MA. Team approach versus ad hoc health services for young people with physical disabilities: a retrospective cohort study. Lancet 2002; 360 (9342): 1280 1286

${ }^{20}$ World Health Organisation. International Classification of Functioning, Disability and Health: ICF: Geneva: WHO; 2001

${ }^{21}$ Stucki G, Ewert T, Cieza A. Value and application of the ICF in rehabilitation medicine. Disability \& Rehabilitation 2002; 24 (17): 932 - 938

22 Brandt E, Pope A (eds). Enabling America: Assessing the Role of Rehabilitation Science and Engineering. Washington, DC: National Academy Press, 1977

${ }^{23}$ Stucki G. Rehabilitation medicine in rheumatic diseases. Current Opinion in Rheumatology 2003; 15: $132-133$

${ }^{24}$ Stucki G, Kroeling P. Principles of rehabilitation. In: Hochberg, Silman, Smolen, Weinblatt, Weisman (eds): Rheumatology $3^{\text {rd }}$ Edition, Vol. 1. Mosby of Elsevier Ltd, 2003: 517-530

25 Stucki G, Ewert T, Cieza A. Value and application of the ICF in rehabilitation medicine. Disability \& Rehabilitation 2002; 24 (17): 932-938

${ }^{26}$ Stucki G, Sigl T. Assessment of the impact of disease on the individual Best Practice \& Research in Clinical Rheumatology 2003; 17 (3): 451 473
${ }^{27}$ Stucki G, Sangha O. Principles of rehabilitation. In: Klippel JH, Dieppe PA (eds): Rheumatology. $2^{\text {nd }} E d n$, Chapter 3. London: Mosby; 1997: $11.1-11.14$.

${ }^{28}$ Medical Rehabilitation for people with physical and complex disabilities. Report of a working party. Journal of Royal College of Physicians of London, 2000

${ }^{29}$ Steiner WA, Ryser L, Huber E, Uebelhart D, Aeschlimann A, Stucki G. Use of the ICF model as a clinical problem-solving tool in physical therapy and rehabilitation medicine. Physical Therapy 2002 Nov; 82 (11): $1098-1107$

${ }^{30}$ Cieza A, Ewert T, Ustun TB, Chatterji S, Kostanjsek N, Stucki G. Development of ICF Core Sets for patients with chronic conditions. Journal of Rehabilitation Medicine 2004 July; 44, Suppl: 9-11

${ }^{31}$ Stucki G, Grimby G. Applying the ICF in medicine. Journal Rehabilitation Medicine 2004 Jul; 44, Suppl: 5-6

${ }^{32}$ Ustun B, Chatterji S, Kostanjsek N. Comments from WHO for the Journal of Rehabilitation Medicine Special Supplement on ICF Core Sets. Journal of Rehabilitation Medicine 2004 Jul; 44, Suppl: 7-8

33 Stucki G, Cieza A. Understanding functioning, disability and health in rheumatoid arthritis - the basis for rehabilitation care. Current Opinion in Rheumatology 2005; 17 (2): $183-189$

${ }^{34}$ UN Standard Rules to provide persons with disability full participation and equality. New York: United Nations, 1994

${ }^{35}$ EU Council of Ministers Meeting. Malaga: European Union, 2003

${ }^{36}$ Rehabilitation and integration of people with disabilities: policy and integration. Strasbourg: Council of Europe Publishing, $7^{\text {th }}$ edition. 2003; 369

${ }^{37}$ A coherent policy for people with disabilities. Recommendation R (92) 6. Council of Europe. Strasbourg, 1992

38 The Standard Rules on the Equalization of Opportunities for Persons with Disabilities (approved by UNO, 20 December 1993). New York: United Nations, 1994

${ }^{39}$ Legh Smith, JA, Denis R, Enderby PM. Selection of aphasic stroke patients for intensive speech therapy. Journal of Neurology, Neurosurgery \& Psychiatry 1987; 50: 1488 - 1492

${ }^{40}$ Warner R. Stroke rehabilitation: benefits of educational initiatives British Journal of Nursing 2000; 9 (20): $2155-2162$

${ }^{41}$ Grahn BE, Borgquist LA, Ekdahl CS. Rehabilitation benefits highly motivated patients: a six-year prospective cost-effectiveness study. International Journal of Technology Assessment in Health Care 2004; 20 (2): $214-221$

42 Turner-Stokes L, Disler PB, Nair A, Wade DT. Multi-disciplinary rehabilitation for acquired brain injury in adults of working age. Cochrane Database of Systematic Reviews 2005; (3): CD004170

${ }^{43}$ Wade DT. Community rehabilitation, or rehabilitation in the community? Disability \& Rehabilitation 2003; 25 (15): 875-881

${ }^{44}$ Turner-Stokes L. The evidence for the cost-effectiveness of rehabilitation following acquired brain injury. Clinical Medicine 2004; 4 (1) $10-12$

${ }^{45}$ Melin R, Fugl-Meyer AR. On prediction of vocational rehabilitation outcome at a Swedish employability institute. Journal of Rehabilitation Medicine 2003; 35 (6): 284-289

${ }^{46}$ Didier JP. La plasticité de la fonction motrice. Collection de l'Académie européenne de Médecine de Réadaptation. Paris: Springer Verlag, 2004: 476

${ }^{47}$ Krauth C, Hessel F, Klingelhöfer HE, Schwelkert B, Hansmeier T, Wasem J. Gesundheitsökonomische Evaluation von Rehabilitationsprogrammen im Förderschwerpunkt Rehabilitationswissenschaften (Health Economic Evaluation of Rehabilitation Programmes in the „Rehabilitation Science“ Research Funding Programme in Germany). Rehabilitation 2005; 44: e46 - e56

48 Boyd LA, Winstein CJ. Impact of explicit information on implicit motor-sequence learning following cerebral artery stroke. Physical Therapy $2003 ; 83$ (11): 976-989

${ }^{49}$ Friberg F, Scherman MH. Can a teaching and learning perspective deepen understanding of the concept of compliance? A theoretical discussion. Scandinavian Journal Caring Sciences 2005; 19 (3): 274 - 279

${ }^{50}$ André JMF. Fondements, stratégies et méthodes en médecine physique et de réadaptation. In: Held JP, Dizien O (eds): Traité de médicine physique et de réadaptation. Paris: Flammarion, 1999: 3-13

${ }^{51}$ SGB IX ( $9^{\text {th }}$ Social law): Rehabilitation und Teilhabe behinderter Menschen. Beck-Texte. Dt. München: Taschenbuch-Verlag, 2001

${ }^{52}$ Garraway GM, Akhtar AJ, Prescott RJ, Hockey L. Management of acute stroke in the elderly: follow-up of a controlled trial. British Medical Journal 1980; 1, 281: 827-829 
53 Mazaux JM, Seze M De, Joseph PA, Barat M. Early rehabilitation after severe brain injury: a French perspective. Journal of Rehabilitation Medicine 2001; 33 (3): 99 - 109

54 Wade D. Investigating the effectiveness of rehabilitation professions a misguided enterprise? Clinical Rehabilitation 2005; 19 (1): 1 -3

55 Quintard B, Croze P, Mazaux JM, Rouxel L, Joseph PA, Richer E, Debelleix X, Barat M. Life satisfaction and psychosocial outcome in severe traumatic brain injuries in Aquitaine. Annales de Readaptation et de Medecine Physique 2002; 45 (8): 456-465

56 McLellan DL. Targets for Rehabilitation. British Medical Journal 1985; 290: 1514

57 Nybo T, Sainio M, Muller K. Stability of vocational outcome in adulthood after moderate to severe pre-school brain injury. Journal of International Psychological Society 2004; 10 (5): 719-723

58 Association of British Neurologists, NeuroConcern Group of Medical Charities, British Society of Rehabilitation Medicine. Neurological Rehabilitation in the United Kingdom. Report of a Working Party. London: British Society of Rehabilitation Medicine, 1992

59 NHS and Community Care Act 1990. London: HMSO, 1990

${ }^{60}$ Hall KM, Cope N. The benefits of rehabilitation in traumatic brain injury: a literature review. Journal of Head Trauma 1995; 10: 1 - 13

61 Verplancke D, Snape S, Salisbury CF, Jones PW, Ward AB. A randomised controlled trial of the management of early lower limb spasticity following acute acquired severe brain injury. Clinical Rehabilitation 2005; 19: 117 - 125

62 Stucki G, Stier-Jarmer M, Gadomski M, Berleth B, Smolenski U. Indikationsübergreifende Frührehabilitation (General early rehabilitation). Phys Med Rehab Kuror 2002; 12: 146-156

${ }^{63}$ Glaesner, JJ, Harloff KJ, Weyer T van de. Rehabilitation im Akutkrankenhaus (Rehabilitation in Acute Hospitals). Fortschritt und Fortbildung in der Medizin Band 29. Köln: Dt. Ärzteverlag, 2005: 13 - 19

${ }^{64}$ Franchignoni F, Salaffi F. Generic and specific measures for outcome assessment in orthopaedic and rheumatological rehabilitation. In: Barat M, Franchignoni F (eds): Advances in Physical Medicine \& Rehabilitation: Assessment in Physical Medicine and Rehabilitation. (ISBN 88-7963-180-2). Pavia: Maugeri Foundation Books, 2004: 58

65 Guzmán J, Esmail R, Karjalainen K, Malmivaara A, Irvin E, Bombardier C. Multidisciplinary bio-psycho-social rehabilitation for chronic low back pain (Cochrane Review). In: The Cochrane Library Issue 4, UK: John Wiley \& Sons Ltd., 2004

66 Karjalainen K, Malmivaara A, Tulder M van, Roine R, Jauhainen M, Hurri $\mathrm{H}$, Koes B. Multidisciplinary bio-psycho-social rehabilitation for subacute low back pain among working age adults (Cochrane Review). In: The Cochrane Library Issue 4, UK: John Wiley \& Sons Ltd., 2004

67 UEMS Charter on Specialty Training EC Directive 93/16/EEC. Brussels. European Commission; 5 April 1993

68 Pradat-Diehl P, Azouvi P. Fonctions executives et rééducation. Paris: Masson, 2006

${ }^{69}$ Azouvi P, Perrier D, Linden M Van der. La rééducation en neuropsychologie. Solal Marseille, 1999

70 Mazaux JM. In: Aphasie. Paris: Masson, 2000

71 Tennant A. Principles and Practice of Measuring Outcome. In: Barat M, Franchignoni F (eds): Advances in Physical Medicine \& Rehabilitation: Assessment in Physical Medicine and Rehabilitation. (ISBN 88-7963180-2). Pavia: Maugeri Foundation Books, 2004: 35

72 Bethoux F, Calmels P. Guide de mesure et d'évaluation en médecine physique et de réadaptation. Paris: Roche, 2003

73 State University of New York at Buffalo. Guide to the use of the uniform dataset for medical rehabilitation (adult FIM). Version 4. Buffalo. NY: State University of New York at Buffalo, 1993

${ }^{74}$ Mahoney FI, Barthel DW. Functional evaluation: the Barthel index. Maryland State Medical Journal 1965; 14: 61 - 65

75 Mau W, Gülich M, Gutenbrunner C, Lampe B, Morfeld M, Schwarzkopf SR, Smolenski UC. Lernziele im Querschnittsbereich Rehabilitation, Physikalische Medizin und Naturheilverfahren nach der 9. Revision der Approbationsordnung für Ärzte. Phys Med Rehab Kuror 2004; 14 : $308-318$

76 Ward AB. Physical \& Rehabilitation Medicine in Europe. Journal of Rehabilitation Medicine 2006; 38 (2): 81 - 86

77 Heilporn A, Andre JM, Didier JP, Chamberlain MA. Violence to and maltreatment of people with disabilities: a short review. Journal of Rehabilitation Medicine 2006; 38 (1): 10-12

${ }^{78}$ National Rehabilitation Guidelines - Italian National Health Service Ministry of Health Official Bulletin, Rome, June 1998

79 SPREAD National Stroke Guidelines - Italian National Health Service. 2001. www.sanita.it

${ }^{80}$ Brain Injury Guideline - Modena and Verona national Consensus Conference - 2001/2005 - www.sanita.it

81 The Department of Health and Social Security: OPCS Surveys of disability in Great Britain Report 1: The prevalence of disability among adults. 1988 (ISBN 0116912294 )

82 Fifty-Eighth World Health Assembly: Resolution 58.23, „Disability, including prevention, management and rehabilitation“. Geneva: World Health Organisation, 2005 


\section{Anhang 1a Vorschlag für eine neue Definition basierend auf} dem ICF-Modell

[Autoren: G. Stucki und J. Melvin in Zusammenarbeit mit dem Professional Practice Committee der PRM-Sektion der UEMS]

Basierend auf dem integrativen Modell der menschlichen Funktionsfähigkeit ist die PRM ein medizinisches Fachgebiet, dessen zentrale Gesundheitsförderungsstrategie die Rehabilitation ist. Es verwendet einen integrierten biomedizinischen und technischen Ansatz, der auf den Fähigkeiten einer Person aufbaut, indem er ihre Eigenressourcen stärkt, für eine supportive Umgebung sorgt und die Fähigkeit der Betroffenen, mit der Umgebung zu interagieren, fördert. Zu diesem Ansatz gehören auch die Diagnostik und Behandlung von Erkrankungen. Die Wirksamkeit der PRM beruht auf:

1. der Beurteilung der Funktionsfähigkeit unter Berücksichtigung vorhandener Erkrankungen sowie persönlicher und umweltbezogener Faktoren einschließlich der Prognose; dem Potenzial, die Prognose zu ändern, der Festlegung längerfristiger Ziele, der Ziele des Interventionsprogramms sowie auf der Feststellung der Rechtsunfähigkeit;

2. der Durchführung und Anwendung biomedizinischer und technischer Interventionen zur Optimierung der Fähigkeit, einschließlich physikalischer Modalitäten, Schmerzlinderungstechniken, neuropsychologischer Interventionen; ernährungsmedizinischer und pharmakologischer Interventionen. $\mathrm{Zu}$ den technischen Interventionen zählen Implantate, Prothesen oder Orthesen, Hilfsmittel und Hilfseinrichtungen zur Stabilisierung, Verbesserung oder Wiederherstellung der Körperfunktionen und -strukturen. Diese führen zu einer Verringerung der Schmerzen, der Müdigkeit und anderer Symptome, beugen Schädigungen, medizinischen Komplikationen und Risiken (wie z.B. Depressionen, Hautulzera, Thrombosen, Gelenkskontrakturen, Osteoporose und Stürzen) vor und kompensieren das Fehlen oder den Verlust von Körperfunktionen und -strukturen;

3. der Leitung und Koordination von Interventionsprogrammen mit dem Ziel, in einem iterativen, multidisziplinären, problemlösungsorientierten Prozess bestmögliche Ergebnisse zu erzielen; auf der Durchführung, Anwendung und Integration biomedizinischer und fachtechnischer Interventionen, psychologischer und verhaltensbezogener Interventionen, aufklärender und beratender Interventionen, ergotherapeutischer und beruflicher Interventionen sowie sozialer und architektonischer Interventionen;

4. der Beratung für Patienten und deren unmittelbaren Umgebung, für Anbieter von Dienstleistungen und für Finanzierungsstellen in der gesamten Dienstbereitstellungskette, d.h. in allen Stadien vom Akutkrankenhaus bis hin zur gemeindenahen Rehabilitation;

5. dem Management der Rehabilitation in Gesundheits- und Mehrzweckeinrichtungen (Multi-Agency-Einrichtungen);

6. der Aufforderung an die Öffentlichkeit und die Entscheidungsträger, entsprechende gesundheitspolitische Maßnahmen und Programme vorzustellen bzw. zu fördern und umzusetzen. - Dies (a) erstreckt sich auf Bereiche, die ein für den Betroffenen erleichterndes, erweitertes physisches, soziales und ökonomisches Umfeld bieten; (b) sichert den Zugang zu Rehabilitationsdiensten als Menschenrecht und (c) befähigt die PRM-Fachärzte, eine zeitgerechte und wirksame Versorgung bereitzustellen, die es den Personen mit Behinderungen bzw. jenen mit einem Behinderungsrisiko ermöglicht, bei der Wechselwirkung mit dem erweiterten physikalischen, sozialen und ökonomischen Umfeld eine optimale Funktionsfähigkeit zu erreichen und zu erhalten.

\section{Anhang 1b Erläuterungen zu den Namensbestandteilen der Physikalischen und Rehabilitativen Medizin}

Die Physikalische Medizin wendet auf wissenschaftlicher Basis alle Interventionen an, die mithilfe physiologischer Mechanismen (z. B. Reflexe, funktionelle Anpassungen und Neuroplastizität) die physiologische und mentale Funktionsfähigkeit verbessern, was auch das physikalische und mentale Training umfasst. All dies wird erst nach einer genauen Funktionsdiagnose mit Hilfe von Funktionstests ermöglicht, die eine der besonderen Kompetenzen der PRM darstellen. Die Physikalische Medizin wird häufig von einem multidisziplinären Team aus Physiotherapeuten, Ergotherapeuten und Mitgliedern anderer entsprechender medizinischer Berufe, Sozialarbeitern, Ausbildungspersonal und Technikern bereitgestellt (siehe Kapitel 5).

Die Rehabilitative Medizin konzentriert sich nicht nur auf die körperliche Funktionsfähigkeit, sondern auch darauf, der betroffenen Person zu helfen, aktiv an der Gesellschaft teilzunehmen. Dies geht auch aus der etablierten Definition der Rehabilitation hervor, die wie folgt lautet: „Ein aktiver Prozess, der Personen, die infolge eines Unfalls oder einer Krankheit Funktionsstörungen erlitten haben, dabei hilft, eine komplette Wiederherstellung zu erreichen, oder der, falls eine Wiederherstellung nicht möglich ist, ihnen dabei hilft, ihr optimales physisches, mentales und soziales Potenzial zu erreichen und in der Lage zu sein, sich in der für sie passendsten Umgebung zu integrieren“ [2]. Beide Möglichkeiten gelten für Personen mit chronischen Erkrankungen, mit Traumata oder mit angeborenen Behinderungen. Zum Erreichen dieses Ziels werden Fähigkeiten wie z.B. Selbstversorgung, Gehen, Auto fahren, Einkaufen gehen, Lernen und viele andere Fähigkeiten in einer Rehabilitationsumgebung trainiert. Eine Übersicht aller möglichen Aktivitäten befindet sich in der Klassifizierungsliste International Classification of Functioning, Disabilities and Health (ICF) [9] (siehe Kapitel 3). 
Anhang 2 PRM-Fachärzte in Europa nach Staaten (die Zahlen entstammen den Angaben der nationalen Delegierten der UEMS-PRMSektion und wurden nicht validiert)

\begin{tabular}{|c|c|c|c|c|c|c|c|}
\hline Land & $\begin{array}{l}\text { Gesamtanzahl } \\
\text { der Ärzte }\end{array}$ & $\begin{array}{l}\text { Gesamtanzahl } \\
\text { der Fachärzte }\end{array}$ & $\begin{array}{l}\text { Anzahl der } \\
\text { Fachgebiete }\end{array}$ & $\begin{array}{l}\text { praktizierende } \\
\text { PRM-Fachärzte }\end{array}$ & $\begin{array}{l}\text { Anzahl der } \\
\text { Auszubilden- } \\
\text { den in PRM }\end{array}$ & $\begin{array}{l}\text { Anzahl der PRM- } \\
\text { Fachärzte/100000 } \\
\text { Bevölkerung }\end{array}$ & Bevölkerung \\
\hline Österreich & 29100 & 13150 & 44 & 134 & 85 & 1,85 & 7300000 \\
\hline Belgien & 38700 & 16365 & 24 & 450 & 80 & 4,40 & 10240000 \\
\hline Kroatien* & 15600 & 8200 & 43 & 299 & 40 & 6,80 & 4600000 \\
\hline Zypern & 2000 & 1850 & 41 & 9 & - & 0,90 & 1000000 \\
\hline Tschechische Republik & 35000 & 27000 & 80 & 483 & 130 & 4,67 & 10300000 \\
\hline Dänemark & 15000 & 6000 & 23 & 120 & 0 & 2,18 & 5500000 \\
\hline Estland & - & - & - & - & - & - & 1350000 \\
\hline Finnland & 19000 & 10500 & 35 & 152 & 30 & 2,92 & 5200000 \\
\hline Frankreich & 183700 & 95000 & 38 & 1760 & 125 & 2,97 & 61300000 \\
\hline Deutschland & 394432 & 261437 & 43 & 1571 & 65 & 1,96 & 80000000 \\
\hline Griechenland & 60700 & 15200 & 37 & 164 & 33 & 1,49 & 11000000 \\
\hline Ungarn & 40829 & 29280 & 92 & 140 & 27 & 1,38 & 10117000 \\
\hline Island* & 900 & - & 33 & 10 & 3 & 3,72 & 270000 \\
\hline Irland & 6050 & 4650 & 51 & 5 & 2 & 0,13 & 4000000 \\
\hline Italien & 307600 & 160000 & 45 & 2200 & 350 & 3,85 & 59000000 \\
\hline Lettland & 8588 & 7437 & 46 & 120 & 7 & 5,42 & 2300000 \\
\hline Litauen & - & - & - & - & - & - & \\
\hline Luxemburg & 810 & 520 & & 8 & 0 & 2,00 & 400000 \\
\hline Malta & - & - & - & 1 & 0 & 0,25 & 400000 \\
\hline Niederlande & 39800 & 16500 & 27 & 248 & 78 & 1,65 & 15000000 \\
\hline Norwegen* & 12800 & - & 28 & 92 & & 2,14 & 4300000 \\
\hline Polen & 109000 & 65000 & 58 & 900 & 120 & 2,30 & 39000000 \\
\hline Portugal & 29950 & 14530 & 46 & 325 & 100 & 3,25 & 10000000 \\
\hline Rumänien* & 44630 & 37670 & 58 & 690 & 164 & 3,14 & 22000000 \\
\hline Serbien u. Montenegro & 25000 & 20000 & 41 & 610 & 120 & 5,80 & 10050000 \\
\hline Slowakei & - & - & - & - & - & - & \\
\hline Slowenien & 4500 & 2830 & 40 & 71 & 16 & 3,55 & 2000000 \\
\hline Spanien & 157900 & 70000 & 51 & 2150 & 292 & 4,89 & 44000000 \\
\hline Schweden & 27000 & 17600 & 60 & 160 & 20 & 1,88 & 8500000 \\
\hline Schweiz* & 28251 & 23170 & 43 & 269 & 50 & 3,60 & 7500000 \\
\hline Türkei* & 91000 & 33000 & 36 & 1300 & 200 & 1,86 & 70000000 \\
\hline Vereinigtes Königreich & 150000 & 21000 & 58 & 129 & 57 & 0,27 & 58000000 \\
\hline
\end{tabular}

* = nicht EU-Mitglied

Anhang 3 Beispiele für zu Behinderungen führenden Erkrankungen, mit denen PRM-Fachärzte häufig konfrontiert sind

\section{Folgen von}

\section{Traumata}

- traumatische Gehirnschädigung,

- Rückenmarkverletzung,

- multiples und komplexes Trauma,

- Plexus brachialis- und periphere Nervenschädigungen,

- Sportverletzungen,

- Schädigungen im Zusammenhang mit einer chronisch behindernden Erkrankung,

- berufsbedingte Verletzungen.

\section{Neurologischen Erkrankungen}

- Schlaganfall - einschließlich Subarachnoidalblutung,
- chronisch behindernde neurologische Erkrankungen wie z.B. multiple Sklerose, Parkinsonsche Krankheit,

- Infektionen und Abszesse des zentralen Nervensystems,

- Tumore des zentralen Nervensystems,

- Spinalparalyse,

- neuromuskuläre Störungen und Myopathien,

- periphere Neuropathien, einschließlich Guillain-Barré-Krankheit und Nervenkompressionssyndrom,

- Alzheimer Krankheit,

- angeborene Erkrankungen - Zerebrale Lähmung, Spina bifida, usw.,

- seltene angeborene Erkrankungen.

\section{Akuten und chronischen Schmerzzuständen}

Amputation (als Folge posttraumatischer oder vaskulärer Erkrankungen sowie bei maligner Erkrankung);

Zustände nach einem chirurgischen Eingriff (einschließlich Organtransplantation), Behandlung auf einer Intensivstation 
(z.B. Intensiv-Polyneuropathie [IP] und Immobilisierungssyndrome [Dekonditionierung] sowie multiples Organversagen).

\section{Erkrankungen des Bewegungsapparates}

- Wirbelsäulenerkrankungen

- akute und chronische Kreuzschmerzen,

- HWS-Syndrome,

- Erkrankung der Brustwirbelsäule,

- degenerative and entzündliche Gelenkserkrankungen,

- sonstige Mono- oder Polyarthritis,

- Weichteilrheumatismus (einschließlich Fibromyalgie),

- komplexe Hand- und Fußprobleme,

- Osteoporose,

- chronische Schmerzsyndrome des Bewegungsapparates,

- arbeitsplatzbezogene Schmerzsyndrome,

- chronische Schmerzsyndrome und Fibromyalgie.

\section{Herz-Kreislauf-Erkrankungen}

- koronare Herzerkrankung,

- Herzinsuffizienz,

- Herzklappenerkrankung,

- Kardiomyopathien,

- periphere Gefäßerkrankung, einschließlich Amputationen,

- arterieller Bluthochdruck.

\section{Atemwegserkrankungen}

- Asthma,

- chronisch-obstruktive Lungenerkrankung,

- Lungenfibrose,

- Staublunge, Asbestlunge und andere industriebedingte Schädigungen.

\section{Fettleibigkeit und Stoffwechselerkrankungen}

- Diabetes mellitus,

- Stoffwechselsyndrome, Hyperlipidämie und Hyperurikämie.

\section{Harnwegserkrankungen}

- chronische Niereninsuffizienz,

- Tumore der Harnwege, einschließlich Prostatakarzinom,

- Prostatabeschwerden (Prostatahypertrophie),

- Inkontinenz - z. B. Stressinkontinenz und postchirurgische Inkontinenz.

\section{Erkrankungen des Magendarmtraktes}

- Morbus Crohn, Colitis ulcerosa,

- funktionelle gastrointestinale Störungen.

\section{Sexueller Dysfunktion (andere als neurologisch bedingte)}

- sexuelle Dysfunktion,

- erektile Dysfunktion.

Infektiösen und immunologischen Erkrankungen

- Knochenmarktransplantation,

- Folgen einer HIV-Infektion.

Krebserkrankungen und ihren Folgen, einschließlich Behandlung von Folgeerkrankungen und palliativer Behandlung

\section{Altersbezogenen Erkrankungen}

- pädiatrische Erkrankungen, einschließlich angeborene Missbildungen, idiopathische Skoliose, Morbus-Perthes, Spina bifida, usw.,

- geriatrische Probleme.

\section{Anhang 4 Diagnostische Methoden und Assessments in der Physikalischen und Rehabilitativen Medizin}

Die Diagnosestellung und Evaluierung in der PRM umfasst folgende Punkte:

\section{Diagnose der Erkrankung}

- Anamnese,

- klinische Untersuchung,

- klinische Diagnosewerkzeuge, z.B. bildgebende Verfahren, usw.

\section{Funktionelle Beurteilung}

- klinische funktionelle Untersuchungen (z. B. Muskeltests, Bewegungsumfang, Koordination, Geschicklichkeit),

- standardisierte/klinische Tests (Timed-up\&go-Test; Functional-Reach-Test, Sit-to-Stand-Test und sonstige),

- technische Tests (Dynamometrie: Muskelbelastungs- und -krafttest, elektrophysiologischer Test usw.),

- Bewertungsskalen und Fragebögen, Ergebnismessungen,

- somatosensorische Tests (Berührung, Temperatur, Druck, Schmerz usw.),

- Körperhaltung, Mobilität, Reichweite, Greifen und Manipulation,

- Testen der Sinnesempfindungen u. spezieller Sinnesfunktionen,

- Schluckfähigkeit und Ernährung,

- Sexualität,

- Kontinenz,

- Lebensfähigkeit des Gewebes (Hautprobleme und Dekubitalulzera),

- Darm- und Blasenfunktion,

- Kommunikation (Sprechen, Sprache und nichtverbale Kommunikation),

- Stimmung, Verhalten, Persönlichkeit,

- allgemeine Gesundheitsparameter etc.

- neuropsychologische Tests (Wahrnehmung, Erinnerungsvermögen, übergeordnete Funktionen, Aufmerksamkeit und sonstige).

\section{Beurteilungen der Aktivität und Teilhabe}

- Anamnese, Checklisten und Fragebögen,

- relevante Umweltfaktoren,

- soziale Situation, Familie und Freunde, Gemeinde,

- berufliche Tätigkeit und Arbeitgeber, finanzielles und sonstiges Vermögen usw.,

- Pflegebedarf,

- Bedarf an Hilfsmitteln (z. B. Rollstuhl),

- Anpassungen der Umgebung (z. B. in der Wohnung).

\section{Spezielle funktionelle Beurteilungen}

Labors für Ganganalyse, mit Spezialeinrichtungen für

- kinematische Maßnahmen - Bewegungsmuster einschließlich zeitlicher und räumlicher Parameter (Goniometrie/Elektrogoniometrie, Akzelerometrie, optoelektronische Systeme, digitale Videokameras mit einschlägiger Software usw.), 
- kinetische Maßnahmen - Bodenreaktionskräfte (dynamometrische Plattformen, pedobarografische Plattformen, Innensohlensensorsysteme, portable Walkwaysysteme usw.),

- Oberflächen-EMG-Aktivität in bestimmten Muskeln,

- Energetik.

Quantifizierung anderer Gleichgewichts- und Mobilitätsaufgaben (statische und dynamische Posturographie, langfristige Aktivitätsüberwachung usw.)

Arbeitsbezogene Leistungsfähigkeit: Tätigkeiten des täglichen Lebens und Haushaltsführung, Arbeit und produktive Tätigkeiten (einschließlich Evaluierung der Funktionsfähigkeit und Untersuchung des Arbeitsstandortes), therapeutische Evaluierung des Fahrverhaltens, Freizeitaktivitäten.

\section{Anhang 5 Studienlehrplan und theoretisches Wissen für ein PRM-Diplom des Europäischen Boards (Version 2005)}

\section{A Spezifisches Basiswissen des Fachgebiets}

Einleitung: Philosophie, Ziele und Methodologie der Physikalischen und Rehabilitativen Medizin.

1. Historische Aspekte des Fachgebiets

2. Behinderung und Rehabilitationsprozess
a) Das traditionelle medizinische Modell,
b) Das Modell der Physikalischen und Rehabilitativen Medi- zin,
c) Rehabilitationsprozess.

3. Physikalische und Rehabilitative Medizin: ein eigenständiges Fachgebiet
a) Kriterien eines eigenständigen Fachgebiets,
b) Philosophie,
c) Ziele,
d) Methodologie.

4. Rolle eines Facharztes für Physikalische und Rehabilitative Medizin
a) Medizinische Aufgaben,
b) Medizinisch-soziale Aufgaben,
c) Ziele und Standards des Fachgebiets,
d) Interdisziplinäre Arbeit.

\section{Kapitel 1 Grundlagen der Physikalischen und Rehabilitativen} Medizin

1. Grundsätze der allgemeinen Biomechanik, sowohl Kinetik als auch Kinematik. Kräfte, Kraftmomente, Hebel, Bewegungen, Leistung, Arbeit, Trägheit, Beschleunigung.

2. Grundsätze des Verhaltens und des Widerstands von Werkstoffen unter Belastung. Allgemeines Verständnis von Belastung und Auswirkungen von Belastung. Merkmale homogener Werkstoffe und Verbundstoffe. Elementare Kenntnisse der Messung von Belastung und Verformung verschiedener Werkstoffe.

3. Biomechanik: allgemeines Verständnis über die Anwendungen der genannten Grundsätze auf lebendes Gewebe. Biomechanik verschiedener Gewebe des menschlichen Körpers (insbesondere des Bewegungsapparates). Elementare Kenntnisse der Biomechanik von Flüssigkeiten und deren Anwendung auf die Flüssigkeiten im menschlichen Körper.

4. Studium der menschlichen Bewegung (Kinesiologie).

a) Allgemein.

Anwendung der Hebelsysteme auf den menschlichen Körper; einzelne Bestandteile der Hebel in Bezug auf den Bewegungsapparat.

Kenntnisse der Gelenkstruktur, Klassifizierung und Merkmale der Gelenkbewegungen.

Muskelkontraktionen, innerer und äußerer Widerstand, Verkürzung von Muskeln und Verringerung des Bewegungsumfangs der Gelenke. Den Bewegungsumfang des Gelenks einschränkende Faktoren. Unterschiedliche Muskeltypen. Unterschiedliche mechanische Arten von Muskelkontraktionen, mono- und polyartikuläre Muskeln. Statische oder isometrische Kontraktionen, dynamische oder isokinetische Kontraktionen.

Isometrische Kontraktionen. Agonisten, Antagonisten, synergetische Muskeln. Kinetische Ketten.

b) Spezifisch.

Physiologie der Gelenk- und Muskelfunktion: Wirbelsäule und Gliedmaßen. Dieser Teil des Programms geht von der Voraussetzung aus, dass der Auszubildende Detailkenntnisse über die Anatomie des Bewegungsapparates besitzt.

c) Angewandt.

Anwendung der oben genannten Daten auf die Analyse der Körperhaltung und -bewegungen bei den grundlegenden Tätigkeiten des menschlichen Lebens: grundlegende Positionen und verschiedene Haltungen, Gebärden, Greifen, Geschicklichkeit, Gehen, Laufen und Springen.

5. Biochemische Auswirkungen der körperlichen Betätigung. Energieaufwand, thermische Regulierung. Physiologische Kosten der Energie, Auswirkungen der körperlichen Betätigung auf das kardiovaskuläre System und die Lungen.

6. Klinische Epidemiologie, Methodologie für die klinische Forschung. Statistik.

7. Evidenzbasierte Medizin im Fachgebiet der Physikalischen und Rehabilitativen Medizin.

8. ICF.

9. Beurteilung der kognitiven Funktion.

\section{Kapitel 2 Physiologie und grundlegende Physiopathologie}

1. Zentralnervensystem,

2. Peripheres Nervensystem,

3. Autonomes Nervensystem,

4. Muskeln,

5. Neuromuskulärer Übergang,

6. Neurophysiologie der Körperhaltung und Bewegung,

7. Schmerzmechanismen, Wechselwirkung von Schmerz und Bewegung.

\section{Kapitel 3. Klinische und funktionelle Beurteilung in der Physikalischen und Rehabilitativen Medizin}

1. Klinische und funktionelle Beurteilung der Gelenke und Muskeln; motorische und sensorische Funktion.

2. Beurteilung von Kognition, Sprechen und Sprache, Erinnerungsvermögen, Verhalten, etc. 
3. Labortests und bildgebende Verfahren. Sinnvoller Einsatz moderner medizinischer bildgebender Verfahren in der Physikalischen und Rehabilitativen Medizin.

4. Quantitative Evaluierung mechanischer oder kinetischer Phänomene (Force Plates) mithilfe der computergestützten Aufzeichnung sowie der Bewegung oder Kinematik (verschiedene moderne Formen der Aufzeichnung von Verschiebungen von Körperteilen).

5. Elektrodiagnose: Elektromyographie, Nervenleitgeschwindigkeitsuntersuchungen und evozierte Potenziale.

6. Lungenfunktionstests.

7. Kardiovaskuläre Funktionstests, Sauerstoffsättigung, stufenweise angeordnete Bewegungstests (Graded-Exercise-Tests), physiologische Energiekosten.

8. Derzeit in der Physikalischen und Rehabilitativen Medizin verwendete biochemische und Pathologietests.

9. Funktionelle Beurteilung: Verwendung und Anwendung der ICF-Klassifikation. Verschiedene Skalen, Instrumente, Tests und Indizes, die bei Erkrankungen verwendet werden, die für die Physikalischen und Rehabilitativen Medizin von Bedeutung sind.

10. Haltungs- und Bewegungsanalyse (einschließlich Posturographie und Ganganalyse).

\section{Kapitel 4 Therapien der Physikalischen Medizin und Rehabilitation}

\section{Physiotherapie}

a) Kenntnis der elementaren Techniken der passiven und aktiven Physiotherapie. Verschiedene Techniken der manuellen und der instrumentellen Massage. Techniken der funktionellen Rehabilitation bei Gelenkproblemen (manuell oder mit Geräten). Muskel(re)training, Stimulation der Muskelaktivität, funktionelles Training. Techniken der Muskelstärkung, des Ausdauertrainings; das Wiedererlernen von Fähigkeiten.

b) Spezifische physiotherapeutische Methoden für einzelne Erkrankungen.

Techniken wie z.B. Cyriax, Mennell usw.

Techniken zur Förderung bzw. Hemmung des neuromuskulären Systems, wie z. B. Kabat, Bobath, Bronström, Vojta usw.

Behandlung von Lymphödemen.

c) Indikationen, Verschreibungen, Kontrollen der Physiotherapie: allgemeine Kenntnisse der Technik und Wahl der Techniken unter Berücksichtigung der Ergebnisse der klinischen und funktionellen Beurteilung, der Art der Erkrankung und der individuellen Eigenschaften des einzelnen Patienten.

2. Modalitäten der Physikalischen Therapie: Verwendung von Techniken der physikalischen Therapie, Grundlagenwissen, Auswirkungen der Behandlung, Indikationen und Kontraindikationen.

a) Elektrotherapie: Galvanische Ströme, Niedrig-, Mittel- und Hochfrequenzbehandlungen,

b) Mechanische Vibration,

c) Biofeedback,

d) Thermotherapie: Therapeutische Kälte- und Wärmebehandlungen,

e) Balneotherapie.
3. Ergotherapie.

Grundlagen der Ergonomie.

Grundlagen und Methoden der Ergotherapie.

Materialien, Geräte, Technologie und Beurteilung auf dem Gebiet der Ergotherapie.

Anwendungen der Ergotherapie: Gelenk-/Muskeltraining, Ausdauertraining, bei funktioneller Rehabilitation und bei der Wiedereingliederung in die Gemeinschaft (gesellschaftlich-familiäres, häusliches, berufliches und Arbeitsplatztraining).

Berufsbezogene Beurteilung, Lenkung und Training.

4. ${ }^{*}$ Geräte und technische Hilfsmittel.

a) Verwendete Materialien,

b) Verbände,

c) Orthosen (Wirbelsäule, Glieder),

d) Prothesen: chirurgische Indikationen zur Amputation. Hilfsmittel zur Rehabilitation für Personen nach Amputation,

e) Stomatherapie,

f) Technische Hilfsmittel und Geräte: Einrichtungen, Werkzeuge, ADL-Hilfsmittel, Transport- und Kommunikationsmittel, Umgebungsoptimierung, Haushaltsgeräte: ob einfach oder anspruchsvoll, wichtig ist, dass sie Personen mit Behinderungen helfen können, ein unabhängigeres Leben zu führen und ihre Lebensqualität zu verbessern,

g) Beratung und Management für Rollstuhlfahrer.

5. Manuelle Therapie

Pathophysiologie der manuellen Medizin. Anatomiekenntnisse. Klinische Untersuchung der Wirbelsäule und der Gliedmaßen. Dysfunktion der intervertebralen Segmente. Grundsätze manueller Techniken. Indikationen und Kontraindikationen. Potenzielle Gefahren bei manipulativen Handlungen. Klinische Untersuchung und Beurteilung.

6. Wiedererlernen bei Sprech- und Sprachstörungen, Grundsätze und Hilfsmittel sowie Technologie der Sprech- und Sprachtherapie. Entwicklung der Sprache im Kindesalter. Beurteilung in der Sprechtherapie und ihre Anwendung auf die Rehabilitation bei Artikulierungs- und Sprachproblemen (Ausdruck, Verständnis; Lesen und Schreiben).

Beurteilung und Behandlung von Schluckbeschwerden.

7. Reintegration von Menschen mit Behinderungen in die Gesellschaft. Grundlagen und Methoden der klinischen Psychologie und sozialen Unterstützung (Sozialarbeit). Kognitive Beurteilung (Verstand, Erinnerungsvermögen, Konzentration, Verhalten) und Lernpotenzial. Anwendung auf kognitive und psychologische sowie soziale Fähigkeiten; Erinnerungsvermögen und Konzentration, Verständnis des Patienten und Akzeptanz seines Problems, Motivation zur Rehabilitation, Stimmung, Schwierigkeiten bei zwischenmenschlichen Beziehungen, berufliche Bildung und Methoden zur Sicherung der finanziellen Sicherheit. Umweltauswirkungen.

\footnotetext{
* Fachärzte für Physikalische und Rehabilitative Medizin sollten allgemeine Kenntnisse über die in Orthosen und Prothesen verwendeten Werkstoffe und deren mechanischen Eigenschaften verfügen. Zusätzlich wird erwartet, dass sie bei häufig verwendeten Werkstoffen Kenntnisse über Herstellungsverfahren, Zubehör und Ersatzteile sowie über deren Anwendung in der Praxis besitzen. Sie sollten genaue Kenntnisse darüber haben, wann und wie sie verschrieben werden sollen, wie sie angepasst werden; sie sollten die Anwendung prüfen und die therapeutischen Wirkungen beurteilen können.
} 
8. Neuropsychologische Rehabilitation: Beurteilung und Behandlung.

9. Pharmakologie: Pharmakokinetik der in der Rehabilitationsmedizin verwendeten Arzneimittel; mögliche Wechselwirkungen mit dem Rehabilitationsprogramm und der Krankengymnastik.

10. Infiltration und Injektionstechniken, Akupunktur.

11. ECSWT: Extrakorporale Stoßwellentherapie bei Sehnenentzündung.

12. Multidisziplinäre Schmerzbehandlung.

\section{B Anwendungen bei speziellen Krankheiten}

Für die nachfolgenden Kapitel ist es nicht notwendig, auf die einzelnen Beschreibungen all jener Erkrankungen, mit denen die PRM-Fachärzte konfrontiert sind, einzugehen. Vielmehr genügt es, wenn die Fachärzte auf dem Gebiet der Physikalischen und Rehabilitativen Medizin die klinischen Zeichen und Symptome, diagnostischen Werkzeuge, entsprechende Beurteilungsmethoden und die aktuellen Therapien für jede Krankheitsgruppe kennen. Von besonderer Wichtigkeit ist allerdings, dass sie die therapeutischen Wirkungen einer Rehabilitation, ihre Indikationen und Kontraindikationen kennen.

\section{Kapitel 5 - Der immobile Patient}

1. Physiopathologie der Immobilisierung. Folgen für das
a) kardiovaskuläre System,
b) respiratorische System,
c) Ernährungssystem,
d) Stoffwechselsystem (Osteoporose),
e) Nieren- und Harnwegsystem,
f) Hautsystem (Haut-Dekubitalulzera),
g) Muskelsystem,
h) den Bewegungsapparat und
i) das neuropsychologische System.

2. Prävention und Behandlung der oben erwähnten Störungen.

\section{Kapitel 6 Erkrankungen des Bewegungsapparates bei Erwachsenen in der Physikalischen und Rehabilitativen Medizin}

1. Nichttraumatische Erkrankungen

a) Entzündliche Gelenkserkrankungen, rheumatische Polymyalgie, Bindegewebserkrankungen,

b) Kristallarthropathien, Gicht, Chondrokalzinose,

c) Osteomalazie, Osteoporose,

d) Paget-Krankheit,

e) Primäre und sekundäre maligne Knochentumore,

f) Extraartikularer Rheumatismus,

g) Neuromuskuläre Erkrankungen,

h) Arthrosen,

i) Erkrankungen der Wirbelsäule,

j) Arthroplastie,

1) Hand- und Fußerkrankungen,

m)Elementare Kenntnisse der Gelenkchirurgie: Arthroskopie, Arthrotomie, Arthrodesie,

n) Chronisches Ermüdungssyndrom und Fibromyalgie,

o) Myofasziales Schmerzsyndrom.

2. Traumatische Erkrankungen bei Erwachsenen a) Physiologie und Pathophysiologie der Wundheilung (Tissue Repair) im Bewegungsapparat,

b) Verstauchungen und Zerrungen,

c) Dislokationen. Traumatische und wiederkehrende Dislokationen,

d) Knochenbrüche: Wirbelsäule (ohne neurologische Komplikationen), Gliedmaßen, Grundsätze spezifischer Behandlungen, Heilungszeit, einschließlich chirurgischer Eingriffe wie z.B. Osteotomie,

e) Bestimmte Erkrankungen der Hände und Füße.

3. Verbrennungen:
a) Klassifikation,
b) Behandlungsverfahren,
c) Vorbeugung und Rehabilitation von Komplikationen.

\section{Kapitel 7 Physikalische und Rehabilitative Medizin und Sport}

Physikalische und Rehabilitative Medizin auf dem Gebiet sportlicher Aktivitäten.

Sport für Menschen mit Behinderungen.

\section{Kapitel 8 Physikalische und Rehabilitative Medizin bei Erkrankungen des Nervensystems}

1. Zentrales Nervensystem

a) Vaskuläre, neoplastische, traumatische, entzündliche, infektiöse und degenerative Erkrankungen des Gehirns und des Rückenmarks, Läsionen des Rückenmarks, traumatische, neoplastische etc. Beurteilung und Behandlung von Rückenmarksläsionen,

i) Spinozerebellare degenerative Erkrankungen: Friedreichsche Krankheit, Strumpell Lorain usw.,

j) Neuropsychologische Dysfunktion,

2. Peripheres Nervensystem

3. Spezifische sensorische Beeinträchtigungen

\section{Kapitel 9 Physikalische und Rehabilitative Medizin bei Erkrankungen der Atemwege}

1. Grundlegende Einschätzung akuter und chronisch obstruktiver sowie restriktiver Syndrome: Ätiologische und therapeutische Grundsätze.

2. Grundlagen der Rehabilitation: funktionelle Beurteilung mit Hilfe von Lungenfunktionstests.

a) Aktive und passive Techniken zur bronchialen und posturalen Drainage, manuelles Abklopfen, instrumentelle Techniken,

b) Patientenaufklärung und -training,

c) Künstliche Ventilation, assistierte Beatmung, assistierte Beatmung zu Hause. Tracheostomiebehandlung,

d) Hilfsmittel für assistierte Beatmung,

e) Überwachung und Beobachtung der Behandlung,

3. Anwendung dieser Grundlagen.

\section{Kapitel 10 Physikalische und Rehabilitative Medizin bei Erkrankungen des Herzkreislaufsystems}

Herzerkrankungen

1 Herzrehabilitation bei Herzklappenerkrankung. 
2 Herzrehabilitation nach Myokardinfarkt.

3 Kardiomyopathien.

4 Herzchirurgie.

Gefäßerkrankungen:

Arterielles System

1. Arterielle Verschlusskrankheit der Beinarterien: Untersuchung: Stellenwert und Technologie der Rehabilitation in den verschiedenen Stadien der Erkrankung.

2. Rehabilitation für Amputierte, Pflege des Stumpfes, Prothetik.

Venöses System

3. a) Prävention und Behandlung tiefer Venenthrombosen,

b) Venenprobleme, Geschwüre, variköse Geschwüre.

Lymphatisches System

\section{Kapitel 11 Physikalische und Rehabilitative Medizin bei Kindern}

1. Beurteilung der Kindesentwicklung: psychomotorische Entwicklung, Bewegung, sensorische und kognitive Entwicklung. Tests und Evaluierung der Entwicklungsstadien.

2. Pathophysiologie des in Entwicklung begriffenen Bewegungsapparates. Wechselwirkung von Wachstum und Anomalien in den Strukturen des Bewegungsapparats und der Gelenke.

3. Neuropädiatrie, Gehirnlähmung, Spina bifida und Myelomeningozele. Frühkindliche spinale Muskelatrophien (SMA). Neuroorthopädische Folgen neurologischer Störungen, Vorbeugung, Behandlung und Beobachtung.

4. Angeborene Missbildungen des Bewegungsapparates (Wirbelsäule, Gliedmaßen, skeletale Agenesie, angeborene Aplasien und Dysplasien; Hüftdysplasie, Klumpfuß, sonstige Fußmissbildungen.

5. Wachstumsstörungen.

6. Längenunterschiede der unteren Gliedmaßen.

7. Idiopathische, angeborene, sekundäre Skoliosen.

8. Kindliche Traumata: allgemeine Beurteilung von Traumata und Rehabilitation bei Kindern, insbesondere bei Schlagverletzungen und Amputationen bei Kindern.

\section{Kapitel 12 Physikalische und Rehabilitative Medizin bei urologischen und sexuellen Problemen}

1. Beurteilung der Physiologie der Blasenkontinenz/Sphinkterkontrolle und der Funktion des Analsphinkters.

2. Pathologien der Blase/des Sphinkters: neurologische und sonstige. Urodynamik. Beurteilung und Behandlung.

3. Beurteilung und Behandlung anorektaler Sphinktererkrankungen sowie der Blasen bzw. Darmhyperaktivität.

4. Sexuelle Folgen neurologischer Fehlfunktionen einschließlich Schwangerschaft bei Spinalparalyse.

\section{Kapitel 13 Physikalische und Rehabilitative Medizin bei älteren Menschen}

1. Alterungsprozess verschiedener Systeme:

a) Zentral- und peripheres Nervensystem: neuronales Altern, Einfluss auf Stimmung, Gehirnfunktion, und Feedbackmechanismen, b) Bewegungsapparat,

c) Kardiovaskuläres und respiratorisches System.

2. Verständnis der Grundlagen geriatrischer Erkrankungen: Epidemiologie, Pathologie, Evolution des Alterns und Therapie.

3. Geriatrische Patienten: Indikationen für den Verbleib in der eigenen Wohnung oder Übersiedlung in ein Pflegeheim.

\section{Kapitel 14 Onkologische Rehabilitation}

\section{Kapitel 15 Wiedereingliederung und Pflege von behinderten und älteren Personen zu Hause}

1. Allgemeines Konzept der Gesundheits- und medizinisch-sozialen Einrichtungen in den einzelnen Ländern der Europäischen Gemeinschaft:
a) Krankenversicherungssysteme Soziale Sicherheit,
b) Krankenhausaufenthalt (privat und öffentlich),
c) Intensive Hauskrankenpflege,
d) Hauskrankenpflege, Pflegepersonal, Heimhilfen usw.,
e) Hilfe und Überwachung über Telefon und sonstige Metho- den der Kommunikation.

2. Kenntnisse der allgemeinen Organisation der Rehabilitation, Stellung und Rolle des Facharztes für Physikalische und Rehabilitative Medizin innerhalb dieser Strukturen.

Anhang 6 Regeln für die medizinische Weiterbildung (CME) und berufliche Weiterentwicklung (CPD) von PRM-Fachärzten

Von jedem vom Board zertifizierten PRM-Facharzt wird erwartet, dass er pro Jahr 50 Punkte sammelt. Diese Vorgabe ist nicht verbindlich, empfohlen wird jedoch, dass jeder Facharzt nach 5 Jahren 250 Punkte besitzt. Zehn Jahre nach der Board-Zertifizierung unterzieht sich der PRM-Facharzt einer auf den CME-Punkten beruhenden Neuüberprüfung und -zulassung: Punkte können wie folgt vergeben werden:

1. Teilnahme an wissenschaftlichen Veranstaltungen:

1 Punkt pro akademischer Stunde der Teilnahme (nicht mehr als 6 Punkte pro Tag) oder

3 Punkte pro Halbtagsveranstaltung oder

6 Punkte pro Ganztagsveranstaltung.

2. Präsentation wissenschaftlicher Arbeiten (Halten von Vorträgen/Präsentieren von Posters):

5 Punkte für jeden gehaltenen Vortrag anlässlich einer approbierten wissenschaftlichen Veranstaltung,

3 Punkte für jedes präsentierte Poster anlässlich einer approbierten wissenschaftlichen Veranstaltung.

\section{Veröffentlichungen in:}

i. Journalen: 10 Punkte für jeden Autor.

ii. Büchern: 10 Punkte für jedes Kapitel.

4. Akademische Tätigkeiten (z.B. Ph.D. (Doktorat) - Einreichung der Dissertation - $\mathbf{5 0}$ Punkte).

\section{Selbstausbildung}

i. Persönlicher Abonnent von Journalen der PRM: 5 Punkte/indexiertes Journal, bis zu 2 Journalen. 3 Punkte, falls Abonnent eines im Index nicht aufscheinenden Journals.

ii. Internet-PRM-Kurse: bei Vorlage des Nachweises: 1 Punkt/Kurseinheit bzw. falls die für die Kurseinheit vergebenen Punkte im elektronischen Programm angeführt sind. 


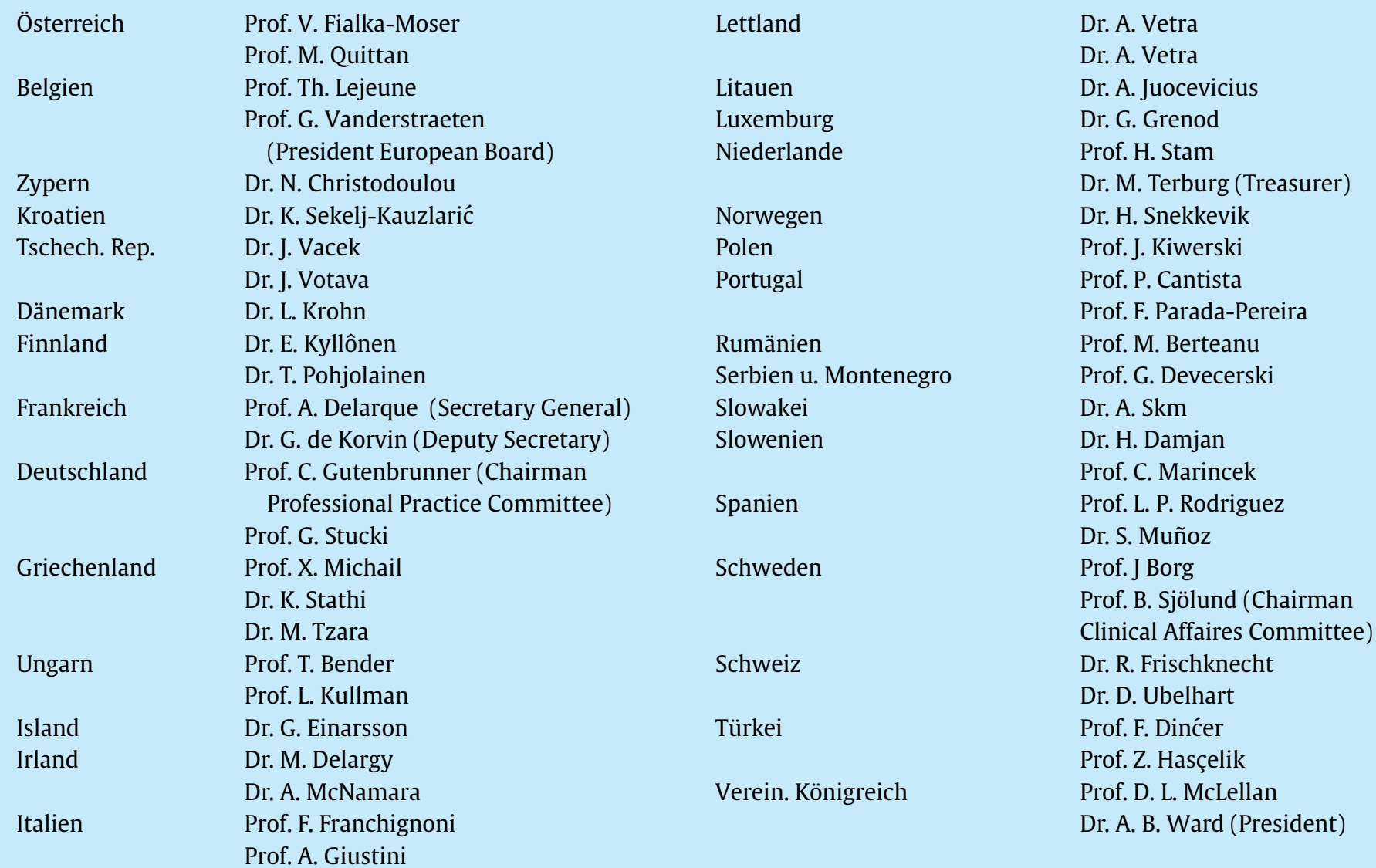

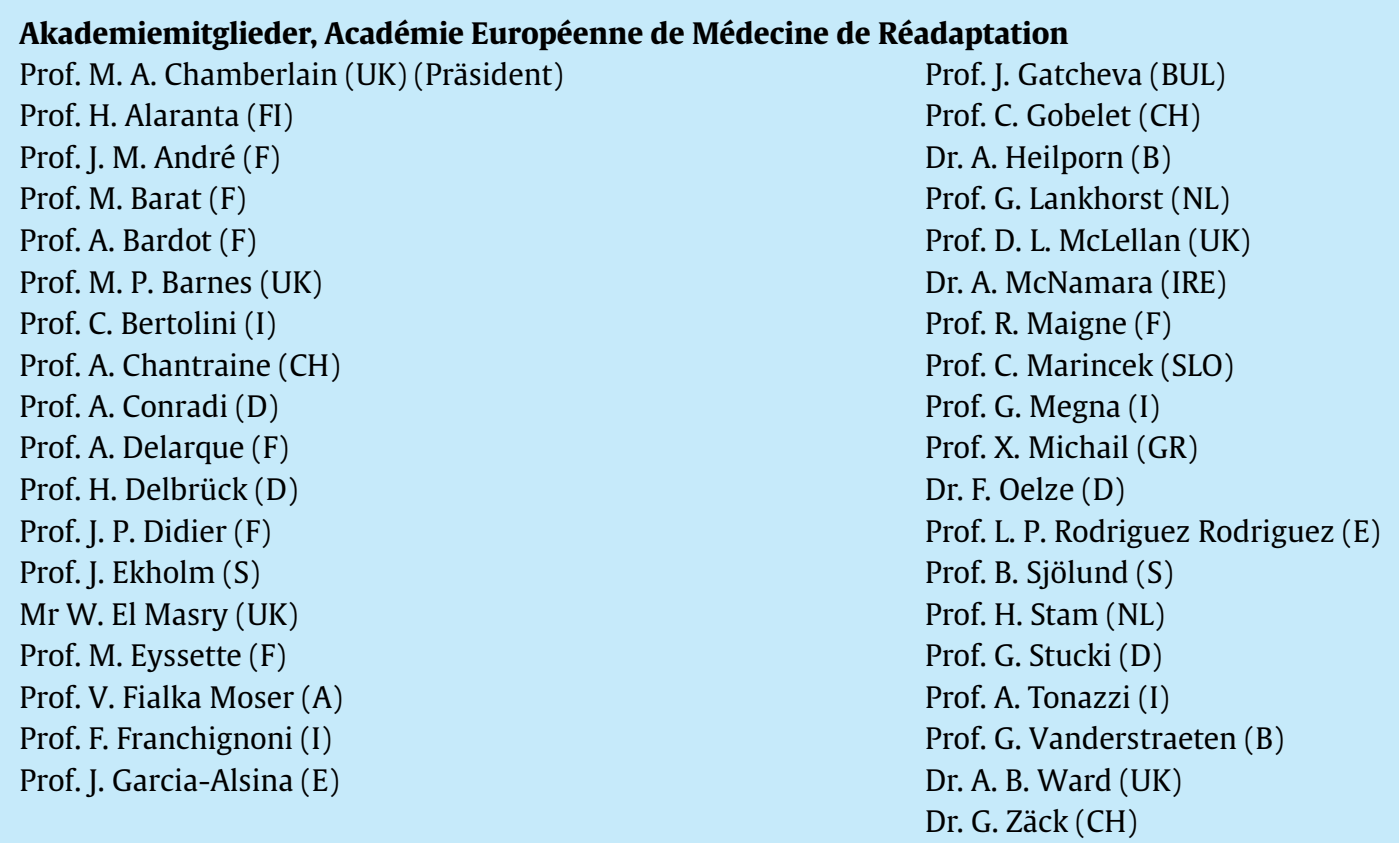

Für die Europäische Gesellschaft für Physikalische Medizin und Rehabilitation

Prof. H. Stam (NL) (Präsident)

Dr. A. Giustini (I) (Vizepräsident)

Prof. X. Michail (Gr) (Vizepräsident)

Prof. G. Vanderstraeten (B) (Vizepräsident)
Dr. A. B. Ward (UK) (Vizepräsident)

Prof. Z. Hasçelik (T) (Sekretär)

Prof. M. Quittan (A) (Schatzmeister) 